Reference: Biol. Bull. 165: 637-685. (December, 1983)

\title{
SURVIVAL, GROWTH, AND BEHAVIOR OF THE LOLIGINID SQUIDS LOLIGO PLEI, LOLIGO PEALEI, AND LOLLIGUNCULA BREVIS (MOLLUSCA: CEPHALOPODA) IN CLOSED SEA WATER SYSTEMS
}

\author{
ROGER T. HANLON, RAYMOND F. HIXON, AND WILLIAM H. HULET
}

The Marine Biomedical Institute, The University of Texas Medical Branch. 200 University Boulevard, Galveston, Texas 77550-2772

\begin{abstract}
Over 1200 squids were captured by night lighting, trawling, or seining in the northern Gulf of Mexico for laboratory maintenance. Two types of recirculating sea water systems were designed and evaluated: a $2 \mathrm{~m}$ circular tank (1500 liter capacity) and a $10 \mathrm{~m}$ long raceway (10,000 liters). Mean laboratory survival was: Loligo plei (12 to $252 \mathrm{~mm}$ mantle length, ML) 11 days, maximum 84 days; Loligo pealei (109 to $285 \mathrm{~mm} \mathrm{ML}$ ) 28 days, maximum 71 days; Lolliguncula brevis ( 27 to $99 \mathrm{~mm} \mathrm{ML}$ ) 19 days, maximum 125 days. Smaller squids showed significantly poorer survival than larger ones. All squids fed well on a variety of live estuarine fishes and shrimps. Growth rates depended upon stage of maturity. The highest rates were Loligo plei $59 \mathrm{~mm} / \mathrm{month}(23.8 \mathrm{~g} / \mathrm{mo})$, Loligo pealei $77 \mathrm{~mm} / \mathrm{mo}(67.3 \mathrm{~g} / \mathrm{mo})$, and Lolliguncula brevis $31 \mathrm{~mm} / \mathrm{mo}(17.2 \mathrm{~g} / \mathrm{mo})$. General aspects of behavior and body patterning were species-specific and were useful indices of the squids' condition. Key factors for laboratory survival were (1) prevention of skin damage, (2) tank systems with sufficiently large horizontal dimensions, (3) high quality water, (4) ample food supply, (5) no crowding, (6) maintaining squids of similar size to reduce aggression and cannibalism, and (7) segregating sexes to reduce aggression associated with courtship, mating, and egg laying.
\end{abstract}

\section{INTRODUCTION}

Pelagic, schooling squids of the Order Teuthoidea are powerful swimmers that forage over great distances in coastal and open-ocean waters. These dynamic predators, with their highly developed organ systems, are of great interest and use to the scientific community, mainly because they have a network of giant axons that mediates a simultaneous contraction of the mantle for jet-propulsed swimming from predators. Historically, researchers have experienced difficulty in collecting and maintaining these animals alive in captivity, due primarily to damage of the delicate squid skin during capture, transport, and maintenance. Over the past ten years, considerable progress has been made in identifying and resolving problems associated with keeping squids alive under laboratory conditions. Since 1975 we have reviewed, tested, and refined many techniques for the capture and maintenance of squids, with the ultimate goal of supplying neuroscience investigators at The University of Texas Medical Branch with live squids. We present here our capture, transport, and maintenance methodology, the design of our closed sea water systems, and we describe the survival, growth, and general aspects of behavior of squids maintained in these systems. 
Three loliginid squid species (Suborder Myopsida, Family Loliginidae) are commonly found on the continental shelf in the northern Gulf of Mexico near Galveston: the tropical arrow squid Loligo (Doryteuthis) plei Blainville, 1823, the common longfinned squid Loligo pealei Lesueur, 1821, and the bay or brief squid Lolliguncula brevis (Blainville, 1823). Aspects of the areal and bathymetric distribution of these species are described by Rathjen et al. (1979), Hixon (1980a) and Hixon et al. (1980). Loligo plei and $L$. peale $i$ in the Gulf of Mexico attain maximal reported sizes of 297 $\mathrm{mm}$ and $285 \mathrm{~mm}$ mantle length (ML), respectively (Rathjen et al., 1979; Hixon, 1980a; Hixon et al., 1980), and they are well-established experimental models, primarily for studies of the giant fiber system (cf., Rosenberg, 1973; Arnold et al., 1974; DiPolo, 1976; Tasaki, 1982). Lolliguncula brevis is a smaller species, maximal $107 \mathrm{~mm}$ ML, that has potential for a variety of scientific applications (Hulet $e t$ al., 1980; Hendrix et al., 1981).

\section{MATERIALS AND METHODS}

\section{Capture}

Field collections were made from two University of Texas research vessels, the $16 \mathrm{~m}$ stern trawler R/V ERIN LEDDY-JONES and the $12 \mathrm{~m}$ R/V VIRGINIA BLOCKER. The R/V ERIN LEDDY-JONES was equipped for bottom trawling and for night lighting with three quartz iodide lamps controlled by rheostats (Fig. 1). One 1000-watt lamp was mounted on the stern A-frame, and two 500-watt lamps were located on either side of the rigging amidships. The R/V VIRGINIA BLOCKER was used for night lighting only. It deployed two portable 500-watt lamps astern or a 500watt underwater mercury vapor lamp.

Great emphasis was placed on obtaining squids by methods that imparted little or no skin damage, particularly to the fins (Hulet et al., 1979). Both species of Loligo were captured alive by attracting them to bright lights at night and dipnetting them onboard. Squid jigs were often used at night-light stations to lure squids to the surface where they were more easily dipnetted. The dipnets were 3 or $5 \mathrm{~m}$-long aluminum poles attached to a $46 \mathrm{~cm}$-diameter stainless steel hoop with a shallow net made of soft $1.3 \mathrm{~cm}(1 / 2$ inch) knotless nylon mesh. Every effort was made to handle the squids briefly and gently. After dipnetting, squids were immediately immersed into a shipboard sea water transport tank so that their water-to-air-to-water transfer lasted only several seconds.

Lolliguncula brevis was captured by bottom trawling and beach seining. Trawl durations were very short (5 to 15 minutes) and in shallow water ( 3 to $10 \mathrm{~m}$ ) in and around Galveston Bay, so that residence time in the net was short and squids were not tightly compressed in the codend for long periods. Forward speed of the vessel was reduced during trawl retrieval and only the codend was swung onboard, placed in water, and the squids quickly placed by hand into transport tanks. Several trawl nets were used, including a 9.1 m-wide (length of foot rope) semi-balloon trawl, a $3.0 \mathrm{~m}$-wide shrimp try net, and $3.0 \mathrm{~m}-, 6.4 \mathrm{~m}$ - or $9.1 \mathrm{~m}$-wide box trawls constructed by Marinovich Trawl Co. (Biloxi, Mississippi). The semi-balloon trawl and the try net were made of $3.8 \mathrm{~cm}$ stretch mesh nylon netting with a codend inner liner of $1.3 \mathrm{~cm}$ mesh knotless nylon netting. The box trawls were constructed entirely with knotless nylon netting ( 1.9 and $1.3 \mathrm{~cm}$ mesh) and were fitted with stainless steel hoops in the codend. Beach seining for Lolliguncula brevis took place at night in summer on the bay side of Galveston Island. Short tows ( 5 minutes) were made with a 30.5 $\mathrm{m}$-long by $2 \mathrm{~m}$-wide bag seine constructed of $1.3 \mathrm{~cm}$ knotless nylon mesh. Squids were transported to the laboratory within one hour of capture. 


\section{Shipboard transport and laboratory transfer}

Squids were always immediately segregated from other captured organisms (e.g., fishes and other invertebrates) and transported in one of three types of shipboard tanks (Fig. 1). The first type consisted of simple, vertically oriented Nalgene cylinders of 200 or 380 I capacity (VT, vertical tank), with no flow-through of sea water. The second consisted of fiberglass cylinders of 380 or $550 \mathrm{l}$ capacity mounted horizontally on skids (HCT, horizontal cylindrical tank) and fitted with a rectangular, hinged opening along the length of the upper surface. These horizontal cylinders substantially reduced sloshing, thereby decreasing haphazard contact between the squids and tank wall. The third type was a 10201 fiberglass rectangular horizontal tank (RHT) with rounded corners. This tank was separated into two layers that doubled the transport capacity over other designs. In the latter two systems, fresh running sea water was pumped into the top and flowed out the bottom. These systems were switched to a recirculating mode and 100 percent oxygen was bubbled into the water when Loligo spp. were being transported from offshore and water quality deteriorated nearshore. Most tanks were covered with polystyrene on the outside for insulation.

For transfer from shipboard to the laboratory, the squids were caught with small dipnets and placed by hand into clear plastic bags $(38 \mathrm{~cm} \times 80 \mathrm{~cm})$, with care being taken not to startle the squids and cause inking. One to three squids and approximately 41 of sea water were put in each bag, which was then pumped full with 100 percent oxygen and tied off. Several bags were placed horizontally in an insulated container, the top was closed, and they were taken by truck several hundred meters to laboratory tank systems with similar temperature and salinity. The bags were floated in the tanks for 15 to 30 minutes until temperatures equilibrated. Each bag was then opened and the squids were released directly into the tank without handling.

Throughout this paper we report our results as mean and median values, but only median values were compared statistically because we used non-parametric tests (see Gibbons, 1976). The shipboard transport, laboratory transfer, and 24 hour acclimation mortality data were analyzed statistically to test for (1) differences in mortality among the three species, (2) differences in the performance of the three tank designs, and (3) differences in mortality associated with squid size. The first comparison (KruskalWallis test) was carried out among all three species, using only the HCT data. The performance of the tank systems was evaluated (Kruskal-Wallis test) using the data of Loligo plei because it was the only species transported in all three tanks. Finally, the third comparison (Mann-Whitney $U$ test) was made between the sizes of $L$. plei that died versus those that survived in the VT and HCT transport tanks; similar data were not available for the other two species.

\section{Closed sea water systems}

A major objective was to develop a large-volume, inexpensive, easily reproducible sea water system that could be modified to test different techniques for maintaining and growing squids. Two basic systems were developed, both being closed systems that recirculated and filtered their own set volume of sea water.

The $2 \mathrm{~m}$ circular tank (CT) system (Fig. 2) is a simple and readily modified design that we developed in 1975 and continues to be our standard system for maintenance and experimentation (Hanlon et al., 1978). Its capacity is approximately 15001 of sea water. Biological filtration, which includes mineralization, nitrification, and dissimilation of nitrogenous compounds ( $c f$., Spotte, 1979a, b), is carried out principally in the filter bed. This layer is $6 \mathrm{~cm}$ deep and consists exclusively of crushed oyster shell (approximate particle size $10 \times 5 \times 2 \mathrm{~mm}$; total weight approximately $160 \mathrm{~kg}$ ) 


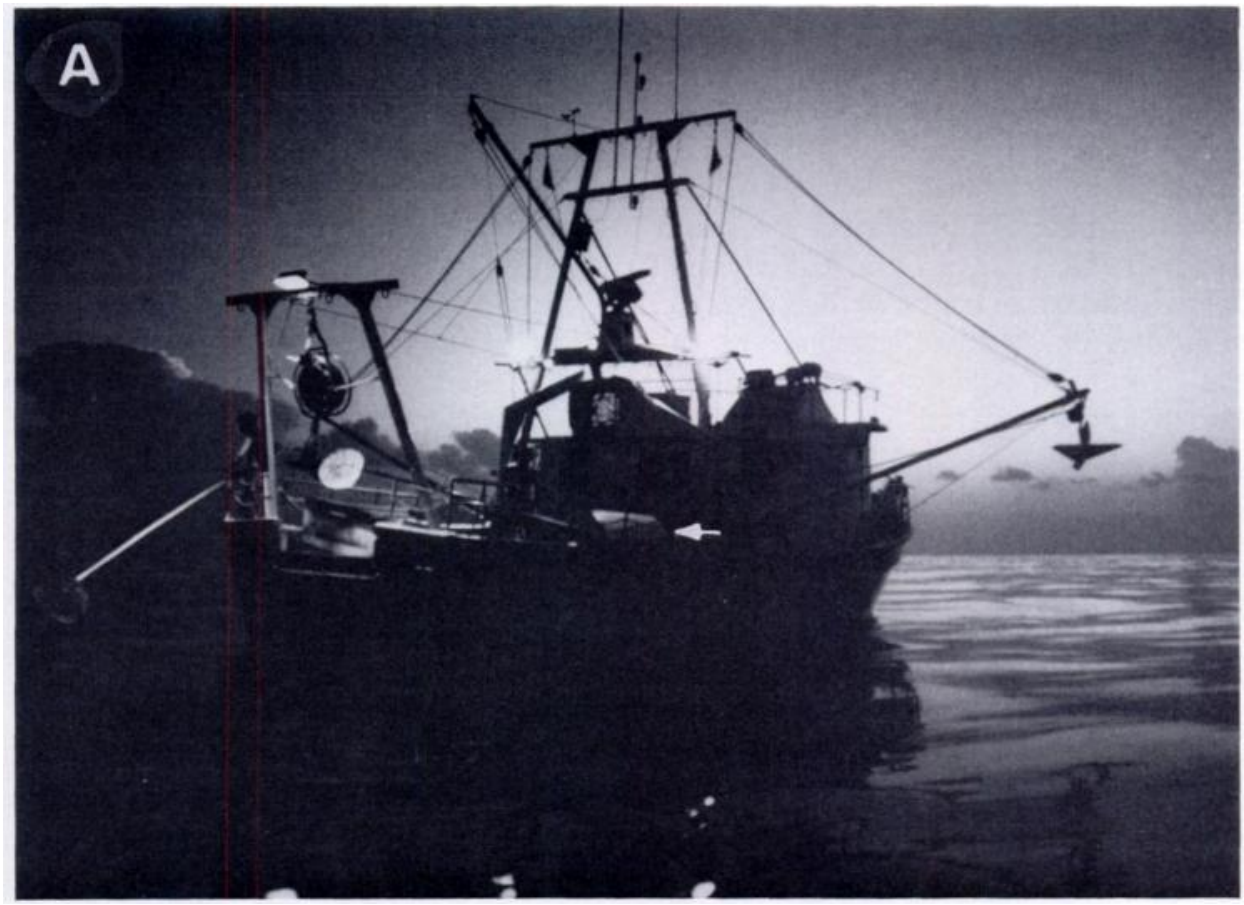

FIGURE 1. Capture and transport. A. R/V ERIN LEDDY-JONES nightlighting for Loligo plei off the coast of Galveston, Texas in $17 \mathrm{~m}$ of water. Note the 1000-watt quartz-iodide lamp on the A-frame and two 500-watt quartz-iodide lamps amidships that are used to attract squids. Squids are dipnetted on board (left) and placed in a transport container (arrow). B. Three types of transport containers: VT is the vertical tank; HCT is the horizontal cylindrical tank; RHT is the rectangular horizontal tank. The tanks and squids are all drawn to the same scale. The squids equal the approximate size of $200 \mathrm{~mm}$ mantle length. Water flow is indicated by arrows. In the RHT, (A) is the removable partition that is replaced when approximately 15 adult Loligo spp. are put in the tank. The tank top (B) is then secured with stainless steel bolts (C) that force a rubber gasket (D) against the top edge of the tank, producing a water-tight seal. Another 15 squids are placed in the upper compartment through the chimney (E). When water quality is good, sea water is continually pumped into the base of the tank through $(F)$ and allowed to overflow from the chimney. When water quality deteriorates near shore, the tank water is circulated by a submersible pump (G) that pushes the water through an exterior filter $(\mathrm{H})$ and back into the tank. Pure oxygen or air may be added through a valve (I).

on which bacteria attach and grow. Newly constructed systems are "conditioned" for several weeks to allow bacterial populations to equilibrate. Toxic ammonia, directly excreted by tank animals or produced indirectly through mineralization of organic products, is oxidized by nitrifying bacteria in the filter bed to nitrite and then to less toxic nitrate. Nitrate is either assimilated by green algae growing in the algal tank under continuous illumination, removed through partial water changes, or removed through dissimilation by bacteria into a completely reduced state in which inorganic nitrogen is released from the water into the atmosphere (Painter, 1970).

Mechanical filtration reduces water turbidity by separating and concentrating particulate organic carbon (i.e., particles, aggregates, detritus, free floating algae, and bacteria) in the filter bed and in two layers of polyester fiber within an auxiliary filter (Fig. 2). Physical adsorption of dissolved organic carbon is accomplished with granular activated carbon in the auxiliary filter or with the periodic use of a foam fractionator 


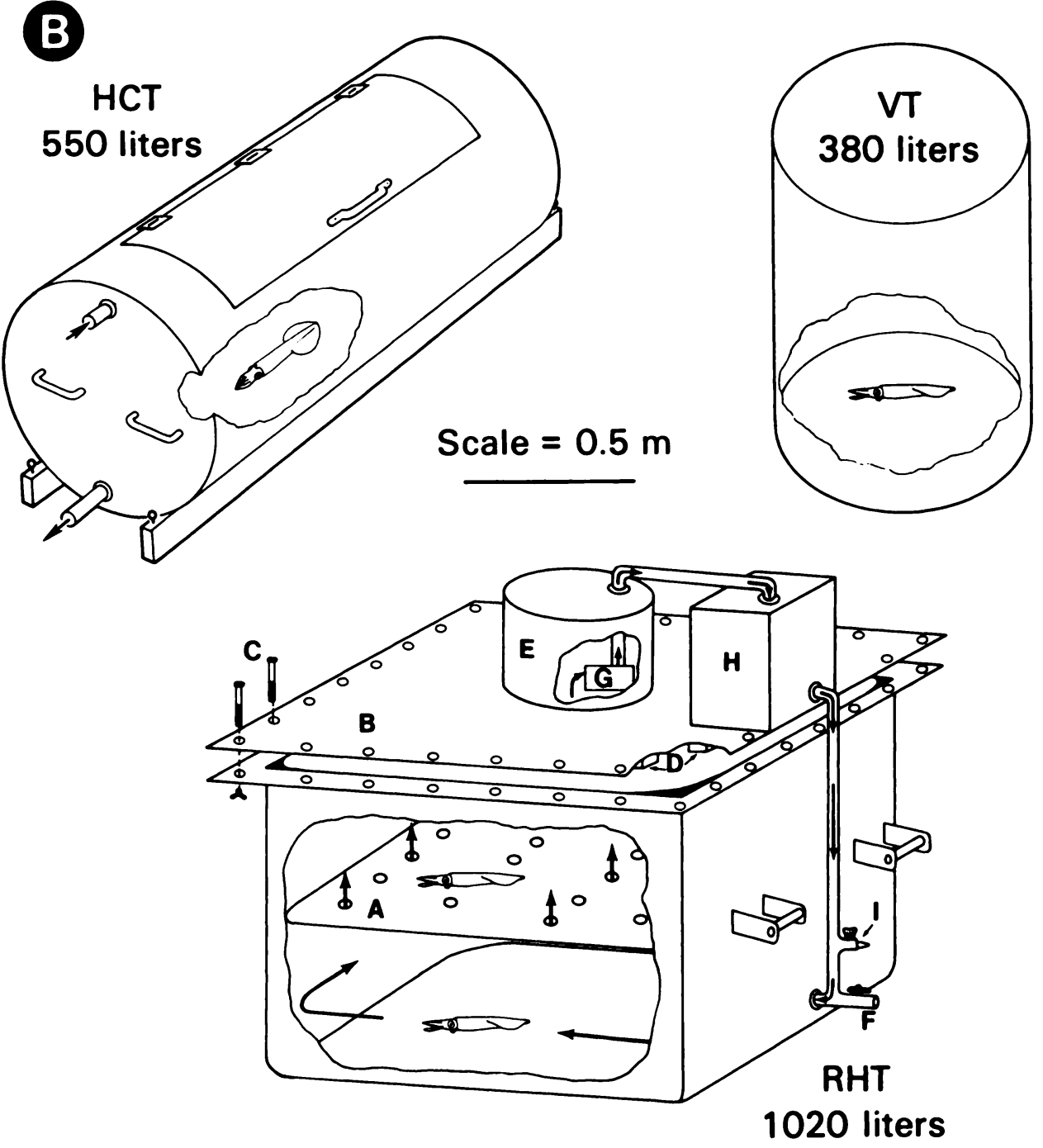

FIGURE 1. (Continued)

or "protein skimmer" that physically binds surface-active organic material to the airwater interface of bubbles and chemically binds non-surface-active compounds with surface-active material (Rubin et al., 1963). This is necessary when the tank is loaded to high capacity and partially eaten food accumulates in the system. Flow rate through the system is approximately 161 per minute.

All fabrication materials are fiberglass, polyvinyl chloride (PVC), or some other inert synthetic product. The only metal components are in the pumps and they do not come in contact with sea water. Tank walls are painted with various patterns made with an inert black paint (Thixochlor, Napko Paint Co., Houston, Texas) to increase contrast and make the walls more visible to squids. Partitions that divided the tank into two or four segments were used occasionally and were constructed of 
R. T. HANLON ET $A L$.

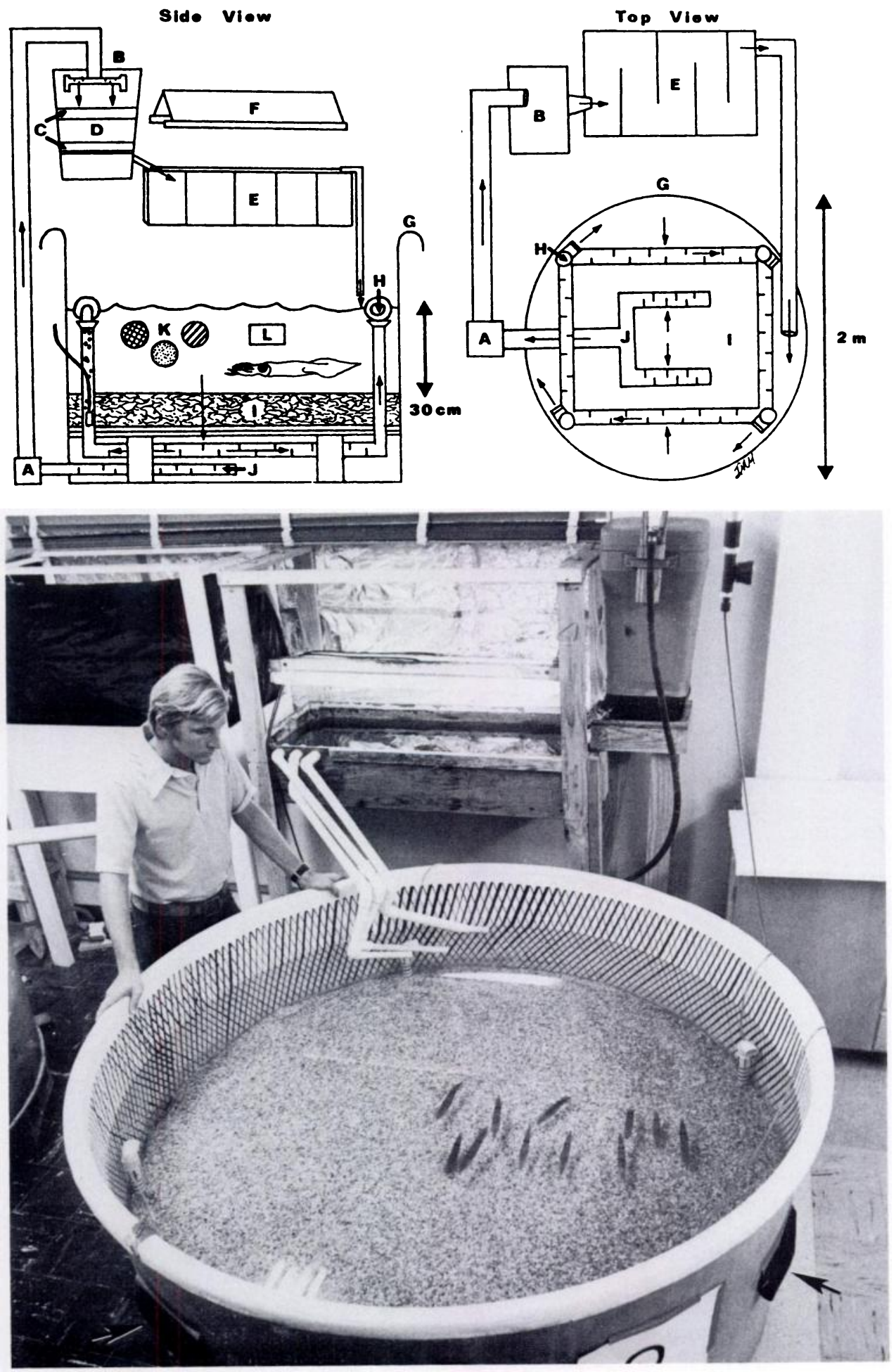


a PVC frame with soft knotless nylon netting. Several CT systems constructed since 1978 have been used without the algal tank.

Our second design is a 10,000 l raceway (RW) system based upon a design for the intensive closed-system culture of penaeid shrimps (Mock et al., 1977). Our original raceway (Fig. 3) was $10 \mathrm{~m}$ long, $2 \mathrm{~m}$ wide, and had rounded ends. The raceway framework consisted of aluminum struts that supported walls made of plywood panels, and rounded ends made of curved aluminum sheeting. A watertight rubberized liner was placed inside the framework, and a corrugated fiberglass partition was suspended lengthwise inside the raceway. Air-lift pumps attached to the central partition provided aeration and water circulation. A CT system (similar in design to Fig. 2) was connected to the raceway to provide water filtration. The algal tank consisted of eight rotating polystyrene "biodiscs" that provided a larger surface area for algal and bacterial growth (Antonie, 1976).

Newer raceways now in operation consist of a single long fiberglass sheet that is curved upward on the sides by supporting struts and closed at each end by a rounded fiberglass half-circle. They may be ordered in a variety of lengths and widths (Ewald Mfg., Karnes City, Texas). The central partition can be omitted to provide greater horizontal space. In this case, water is pumped through auxiliary filters (similar to those shown in Fig. 2, part B) and re-enters the raceway to provide aeration and directional water flow.

When necessary, water is chilled by cooling units (Model D1-100, Frigid Units, Inc., Toledo, Ohio). A deionized water unit provides water for mixing artificial sea water and for replacing water lost through evaporation. Polystyrene panels are fitted over the tops of the raceway and the CT system. These covers reduce evaporation, provide some temperature insulation, and prevent outside activity from disturbing experimental animals.

Both natural and artificial sea water (Instant Ocean Brand, Aquarium Systems Inc., Eastlake, Ohio) have been used in our tanks. Water quality was monitored frequently. Temperature, salinity, and $\mathrm{pH}$ were recorded every one to three days. Estimates of inorganic nitrogen buildup were made biweekly with field test kits (Hach Chemical Co., Ames, Iowa) and precise measurements were made periodically for ammonia (Solórzano, 1969), nitrite (Strickland and Parsons, 1972) and nitrate (Rand et al., 1976). No tolerance levels for these ions have been established for cephalopods, but a partial water change was made when the concentrations exceeded those recommended for most marine animals (Spotte, 1973, 1979a, b). At approximately monthly intervals, a trace element mix (Wimex Trace Elements, Hawaiian Marine Imports, Houston, Texas) was added to each system to replenish those trace elements lost through algal metabolism. Dissolved oxygen measurements were made infrequently, but were always near saturation. Activated carbon in the auxiliary filters was changed every four to ten weeks, depending upon the animal load in the system. The foam fractionators and UV sterilizers were used continuously. Lighting was from indirect natural sunlight and from overhead fluorescent lights regulated to provide a natural light/dark photoperiod.

FIGURE 2. The $2 \mathrm{~m}$ circular tank (CT). This closed sea water system is shown with 11 female Loligo plei. A pump (A) pushes water to an auxiliary filter (B), where it then flows by gravity through two layers of polyester fiber (C) and granular activated carbon (D) into an algal tank (E) that is under continuous illumination (F) and back into the squid holding tank (G). Water circulation in $G$ moves in a clockwise direction that is caused primarily by the flow from air-lift pumps $(\mathrm{H})$. Water is drawn through the filter bed (I) into the perforated subsurface pipes of the air-lift pumps (H). Water is also drawn into another set of subsurface pipes $(\mathrm{J})$ by the pump $(\mathrm{A})$. Various painted patterns $(\mathrm{K})$ make the wall more visible to the squids. Viewing ports ( $\mathrm{L}$, and arrows in photograph) are used for underwater observations. 

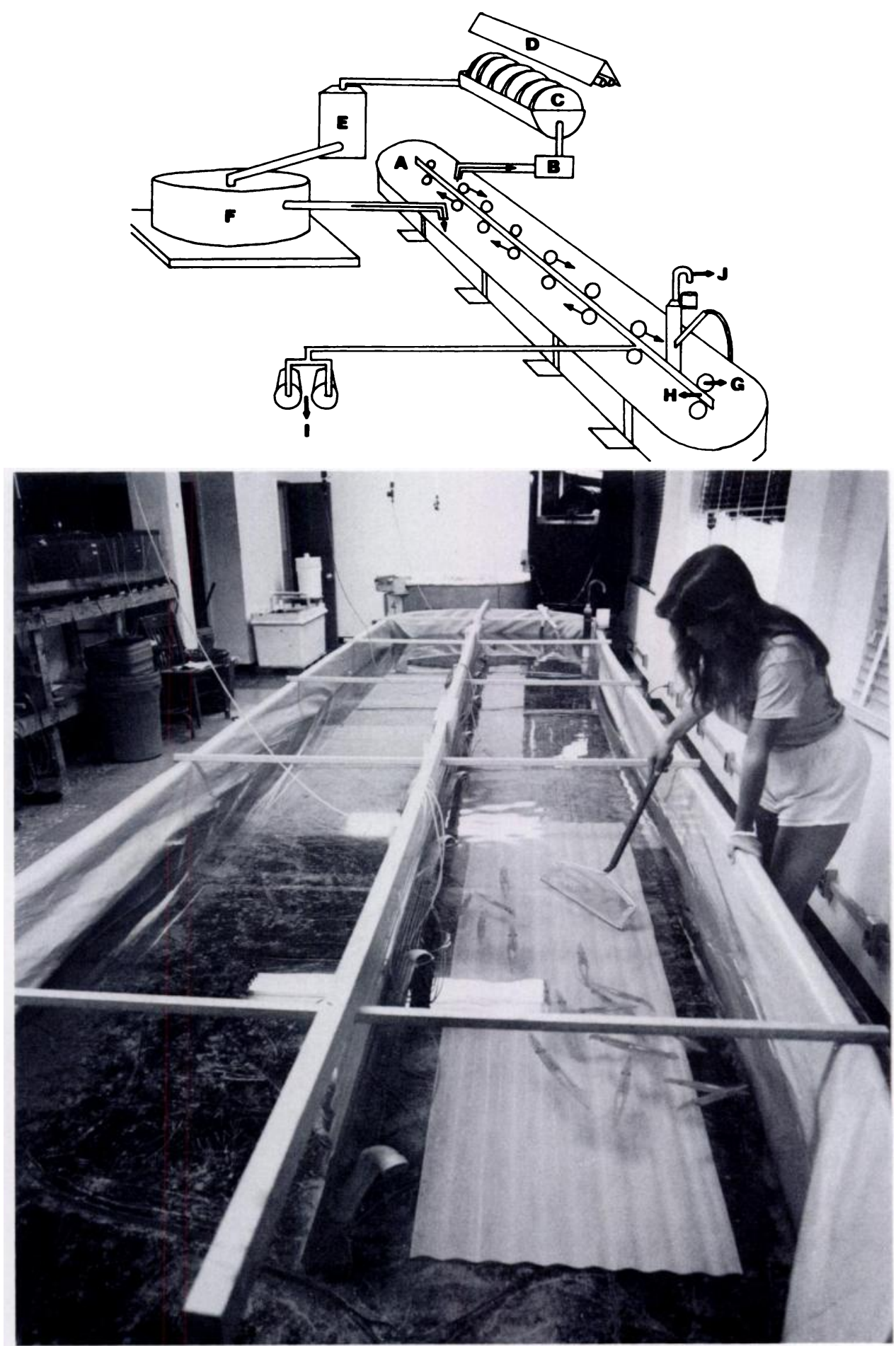


\section{Recapturing, handling, and anaesthetizing live squids}

Squids could be dipnetted from both tank systems due to the narrow dimensions of each. It was usually possible to slowly herd the schools into particular sections of the tank and then isolate individuals for netting (Fig. 3). Dipnetted animals were handheld and gently immersed into a solution of 1.0 to 1.5 percent ethanol in sea water for one to three minutes until respiratory movements slowed or stopped. Squids occasionally inked in the ethanol solution, and the ink was immediately dipnetted out with a fine-mesh net. The anaesthetized animals could be examined, weighed, or measured for a period of five to ten minutes. Thereafter, each squid was placed by hand into sea water and rocked to and fro for 30 to 180 seconds until it regained alertness and body control and swam off. Squids released directly into the tank before full recovery were often attacked by other squids.

\section{Survival, growth, and mortality analyses}

When squids were maintained, daily records were kept for each laboratory tank system. When each squid died, the date of death, number of days since capture, sex, mantle length, stage of sexual development, and probable cause of mortality were recorded. Data on mantle length, sex, and sexual development were unavailable when squids died from cannibalism or when squid remains were eaten by food organisms in the tank. Small-sized, usually immature, squids less than $40 \mathrm{~mm}$ ML in Lolliguncula brevis and less than $50 \mathrm{~mm}$ ML in Loligo plei were termed "juveniles." The analysis of variance procedure by ranks (Conover and Iman, 1976) was used to detect differences in laboratory survival time observed among (1) the three species and (2) males, females, and juveniles within each species.

For determinations of growth of laboratory animals, squids were maintained in the CT systems. Individuals that were in good condition after one week in captivity were measured at intervals of 7 to 21 days. After being anaesthetized, each squid was (1) held head-down for several seconds to allow the mantle cavity to drain, (2) gently blotted on absorbent paper towels, (3) sexed, (4) weighed to the nearest gram, and (5) measured (dorsal mantle length) to the nearest $\mathrm{mm}$. No attempts were made to mark squids for identification, but notes were taken of recognizable differences in individuals (e.g., scars, damaged chromatophores) and this was sufficient to identify squids in subsequent examinations. Throughout the growth observations, palaemonid shrimps and various small cyprinodont fishes were fed to the squids at least twice daily. There was an excess of live food in the tanks at all times. Male and female squids were segregated in the Loligo spp. observations but not in those of Lolliguncula brevis.

Increases or decreases in mantle length or wet weight over the duration of the growth observation were expressed in two ways: (1) as the change in mantle length or wet weight per month (30 days; abbreviated mo), and (2) as an instantaneous

FIGURE 3. The 10,000 I raceway (RW), a closed sea water system. Seventeen Loligo plei, mostly males, are seen swimming over a white fiberglass sheet (put in for the photograph only). The air-lift pumps are turned off for the photograph. Water leaves the raceway (A) via a siphon to a pump (B) that pushes the water to a rotating biodisc tank (C) that is under continuous illumination (D). The water flows by gravity first into the auxiliary filter (E) then through the main filter $(F, a C T$ system) and then back to the raceway. Water within the raceway is circulated in a clockwise direction by the discharge from the main filter and by the air-lift pumps. Two air blowers (I) drive the air-lift pumps. A foam fractionator (J) is mounted in the raceway. Note the accessibility of the squids for recapture. 
relative growth rate expressed as the percent gain in length or weight per day (Winberg, 1960). Statistical analyses were conducted only with Lolliguncula brevis, and using only monthly changes in mantle length and wet weight; no statistical comparisons were made with Loligo spp. due to the small sample size. Tests were made on Lolliguncula brevis to compare the monthly growth rates of males and females (MannWhitney $U$ test), and to detect size-dependent differences in growth rate within each sex (Kruskal-Wallis test).

Two six-day observations were conducted with six Loligo plei to collect preliminary data on gross growth efficiency (or food conversion efficiency). This was measured as the ratio $G / I \times 100$, where $G$ was wet weight increase of the squid and I was total wet weight of food ingested. Only fishes were used as food. All fishes were weighed before entry into the tank (twice per day) and their remains removed and weighed daily. The ingested wet weight of fish was calculated simply by subtracting the total weight of food remains from the total weight of fishes.

\section{Feeding}

Daily feeding consisted of small live estuarine fishes and shrimps. Principal food organisms included the sheepshead minnow Cyprinidon variegatus, the longnose killifish Fundulus similis, the diamond killifish Adinia xenica, the sandtrout Leiostomus xanthurus, the sailfin molly Poecilia latipinna, the tidewater silverside Menidia beryllina, juvenile mullet Mugil spp., juvenile menhaden Brevoortia spp., juvenile and adult penaeid shrimp Penaeus spp., and the palaemonid shrimp Palaemonetes pugio. These species were all readily seined throughout the year in nearby salt marsh areas, estuarine bay waters, and low-energy beachfronts. These organisms are part of the natural diet of Lolliguncula brevis, but not of Loligo plei or L. pealei, which come from offshore. In most cases food was dropped into the tank two or three times per day in quantities that allowed feeding ad libitum throughout the day and night. Most prey organisms were equal to or slightly greater than the length of the squids' arms, but on some occasions mid-sized squids attacked and ate prey organisms nearly their own length. Food remains were netted or siphoned out of the tanks daily.

\section{Behavioral observations}

In the laboratory, squids were observed carefully and often from above the tanks or, more commonly, through the windows in the tank walls. In order to determine possible direct and indirect causes of mortality, particular attention was paid to chromatophore patterning, postures, and general aspects of behavior associated with temperature or salinity shock, fin damage, feeding, and intraspecific interactions.

In the field, Loligo spp. were observed occasionally by skin or SCUBA diving near the boat during night lighting stations offshore from Galveston and throughout the western Gulf of Mexico. More extensive night diving observations were made on Loligo plei in St. Croix in 1978 (Hanlon et al., 1980) and at Grand Cayman Island in 1980 (Hanlon and Hixon, 1981).

\section{RESULTS}

\section{Capture}

For the analyses of shipboard transport and laboratory maintenance, a total of 700 Loligo plei and 89 Loligo pealei were captured by night lighting and dipnetting, 
and approximately 425 Lolliguncula brevis were captured by bottom trawling. Many more squids were obtained during these collections between 1976 and 1982, but they were preserved for other studies. A wide size range of animals was collected in the northern Gulf of Mexico for these analyses: Loligo plei 12 to $252 \mathrm{~mm} \mathrm{ML;} \mathrm{Loligo}$ pealei 109 to $285 \mathrm{~mm} \mathrm{ML}$; and Lolliguncula brevis 27 to $99 \mathrm{~mm} \mathrm{ML}$.

Capture results for each species varied with season, year, and collection site. The areal and bathymetric distributions of the three species near Galveston have been outlined previously (Rathjen et al., 1979; Hixon et al., 1980), and Hixon (1980a, b) has described aspects of the seasonal movements and abundance of each species. At present, we estimate the seasonal availability of each species as follows. Loligo pealei is generally present on the edge of the continental shelf ( 40 to $250 \mathrm{~m}$ deep), but this species is more abundant in fall, winter, and spring and less abundant in summer. Loligo plei is present closer to shore in depths between 20 and $75 \mathrm{~m}$. Large adults are most abundant in spring and summer. Small and mid-sized $L$. plei are found farther offshore during fall and winter, but large adults disappear from the northwestern Gulf in early fall and do not reappear until spring. Lolliguncula brevis is present yearround. It is usually abundant in Galveston Bay ( 1 to $20 \mathrm{~m}$ deep) between early spring and late fall. When bay temperatures drop in winter, this species moves out of the bay to nearshore waters less than $40 \mathrm{~m}$ deep along the Texas coast.

Both species of Loligo came to night lights, but Loligo plei did so more readily and consistently than Loligo pealei. We conducted 164 night light stations for these species totaling 301 hours of observations. Capture rates by dipnet were low for both species: 5.0 squids/h for $L$. plei and 0.9 squids/h for $L$. pealei. Occasionally there were highly productive nights in which hundreds of squids could be easily dipnetted; on these nights capture was terminated quickly when onboard tanks were filled, so the capture rates are conservative. Other contributing factors to the low numerical catch rate were that: (1) squids were often present, but out of dipnet range, (2) very small squids were often not collected, and (3) there were seasonal and yearly decreases in squid abundance and many of these observations were taken during year-round exploratory fishing.

The quartz-iodide lamps deployed above water generally attracted more squids than the underwater mercury vapor lamp, but our attempts to quantify this observation have failed (e.g., Hanlon et al., 1980; Hanlon and Hixon, 1981). Changing the light intensity to draw in squids seen on the periphery of the lighted area did not work consistently. Some squids were caught with squid jigs, but usually jigs attracted squids near the boat for dipnetting. Thus far, no particular style of jig has been effective for consistently capturing these species of Loligo, although a wide variety of jigs from Japan and South America has been used (cf., Rathjen et al., 1979, Fig. 4).

Trawling and seining have been reasonably successful capture methods for $\mathrm{Lol}$ liguncula brevis. The slow-moving nets were effective because this species lives in nearshore waters of high turbidity, thus reducing net avoidance. We believe that the large trawls $(6.4$ and $9.1 \mathrm{~m})$ caught higher numbers of Lolliguncula brevis than the small trawls $(3.0 \mathrm{~m})$, but comparisons could not be made because of the wide variability in the seasonal and yearly use of the nets and differences in the abundance of the animals.

Vanous injuries were sustained by the squids during capture, and these affected their subsequent survival during transport and later in the laboratory maintenance tanks. Loligo plei and Loligo pealei caught with dipnets were practically unharmed when placed in the onboard transport tanks. Squids caught with jigs had small puncture wounds in the arms, tentacles, and funnel, but no permanent damage was done to the skin on the mantle and fins. In comparison, most of the squids caught by the 
trawls sustained skin abrasion caused by the net or other captured animals. The use of nets such as box trawls or beach seines, which are constructed entirely of knotless nylon netting, may reduce skin abrasion caused by the knots in conventional nets. Survival in the trawls was poor when squids were caught with stinging jellyfishes or organisms with hard or pointed exoskeletons such as crabs. Squids generally survived capture better when caught with moderately large numbers of small schooling fishes such as anchovies or menhaden.

\section{Shipboard transport and laboratory transfer}

Success in shipboard transport varied greatly depending upon the species caught, the squids' size, and physical condition after capture, time in transport, sea and weather conditions, and type of shipboard transport tank (Tables I, II, and III). Mortality associated with shipboard transport and laboratory transfer included squids that died any time from capture through their first $\mathbf{2 4}$ hours of acclimation in the laboratory tank systems. Average mortality was 35 percent for Loligo plei during a mean transport time of 7 hours (standard error of the mean, $S \bar{x}, 1.1$ hours). Average mortality was 48 percent for Loligo pealei during a mean transport time of 15 hours $(\mathrm{S} \overline{\mathrm{x}}=3.2$ hours). Average mortality was 27 percent for 324 Lolliguncula brevis during a mean transport time of 1 hour $(S \bar{x}=0.4$ hours). However, no statistically significant differences were found in median mortality (L. plei, 17.5 percent; $L$. pealei, 33 percent; Lolliguncula brevis, 29 percent) among the three species when transported in the HCT. Most mortality in Loligo plei occurred in small squids less than $50 \mathrm{~mm} \mathrm{ML}$, some of it due to cannibalism by larger squids. High mortality in Loligo pealei was attributable to the long transport times and the relatively small horizontal tanks (380 and $5501 \mathrm{HCT}$ ) in which this large species was transported. In contrast, Lolliguncula brevis had the shortest transport time and low mortality; a contributing factor was that mortality rates associated with beach seining (Table III, Observations $9,10,11$, and 12 ) were between only 0 and 13 percent.

Mortality in the vertically oriented cylinders (VT) was high compared to the horizontal cylindrical tank (HCT) or the rectangular horizontal tank (RHT). When mortality of all squids of all three species was compared by type of transport tank, overall pooled mortality in the vertical tanks was 47 percent versus 28 and 24 percent in the other tank designs. For Loligo plei, transport in the vertical tanks resulted in 53 percent overall pooled mortality versus 20 and 24 percent in the HCT and RHT tanks, respectively (Table I). However, for this species no statistically significant differences in median mortality (VT, 33 percent; HCT, 17.5 percent; RHT, 16 percent) were found among transport containers (Kruskal-Wallis test, .05 $<P<.10$ ). Nevertheless, we found the vertical tanks unacceptable because of the lack of flowing sea water and because their narrow horizontal dimensions led to crowding, uncontrolled water sloshing, and fin and skin damage due to collisions with the tank wall.

The horizontally oriented cylinders and the rectangular tank functioned better than the vertical tanks. The closed tops in both designs substantially reduced sloshing, thereby decreasing haphazard contact between the squids and the tank walls. When sea conditions were good, squids swam in the middle of the water column or slightly nearer the bottom; in general, the upper half of the water column was unused by the squids. The 10201 rectangular horizontal tank successfully utilized this upper part of the water column by insertion of a horizontal divider after a number of squids had already distributed themselves across the bottom of the tank. The next batch of squids was then collected and placed in the upper level.

Small-sized squids did not withstand capture and transport as well as larger con- 
specifics. For example, Loligo plei that survived transport in the HCT were significantly (Mann-Whitney U test, $P<.001$ ) larger (median ML $113 \mathrm{~mm}$ ) than squids that died during transport (median ML $53.5 \mathrm{~mm}$ ). Similar, but not statistically significant, results were found in $L$. plei transported in the VT. Smaller squids were generally more damaged during capture, they incurred more skin damage from wall contact during transport, and adult Loligo often cannibalized smaller squids in the same transport tank.

Transferring squids in plastic bags to the laboratory was successful. Although somewhat time consuming, it insured that each squid had adequate sea water, oxygen, and space during this critical period. There was also little sloshing, and when there was sloshing the rounded sides of the horizontally oriented bags reduced fin abrasion. The squids transferred well in the darkness of the closed container. This served to cut off their view of all external commotion, to which they reacted poorly. It was important not to jar the squids during this process. All movements were gentle including driving, closing truck doors, and carrying the squids into the laboratory; otherwise the squids were startled and would ink in the bag.

Healthy, undamaged squids of all three species were able to survive substantial temperature and salinity changes between capture and release into the laboratory tanks. During transport, temperature and salinity usually changed slightly from conditions at the capture sites. When the squids were transferred to the laboratory maintenance tanks in plastic bags, temperature equilibration usually took place within $\mathbf{3 0}$ minutes, whereas salinity changes occurred abruptly when the squids were released into the tanks. These changes in temperature and salinity are listed for each species in Tables I, II, and III.

Lolliguncula brevis (Table III) was subjected to the largest temperature and salinity changes. The largest temperature changes were +11 or $-9^{\circ} \mathrm{C}$ (average change was approximately $\pm 6^{\circ} \mathrm{C}$ ), and the largest salinity shocks were +12 or -8 ppt (average shock was slightly less than $\pm 5 \mathrm{ppt}$ ). The combination of most extreme change was in Observation 1, with a salinity decrease of $8 \mathrm{ppt}$ combined with a temperature decrease of $9^{\circ} \mathrm{C}$. As expected, this estuarine nearshore species tolerated salinity and temperature changes quite well. Hendrix et al. (1981) have recently analyzed salinity tolerance in this squid and shown that this species is an osmoconformer that readily moves within salinities between 17 and 36 ppt.

Loligo plei and Loligo pealei tolerated surprisingly large changes with little or no apparent harm to their subsequent laboratory survival. In L. plei (Table I), the largest temperature changes were +8 or $-11^{\circ} \mathrm{C}$ (average change approximately $\pm 5^{\circ} \mathrm{C}$ ), and the largest salinity shocks were +9 or -8 ppt (average shock was about $\pm 3.5 \mathrm{ppt}$ ). The combination of most extreme change was in Observation 17, with a salinity increase of $7 \mathrm{ppt}$ and a concurrent temperature decrease of $11^{\circ} \mathrm{C}$. For L. pealei (Table II), the largest temperature changes were +3 or $-8^{\circ} \mathrm{C}$ (average change approximately $\pm 5^{\circ} \mathrm{C}$ ), and the largest salinity shocks were +4 or -5 ppt (average shock was about +2 ppt). The combination of most extreme change was in Observation 2, with a salinity increase of $4 \mathrm{ppt}$ and a temperature decrease of $8^{\circ} \mathrm{C}$. Presumably the salinity changes were dealt with by equilibrating blood osmolality through volume regulation, as found in Lolliguncula brevis (Hendrix et al., 1981).

It was very difficult to detect any deleterious effects of these physiological stresses. Even in the extreme cases cited above, most of the undamaged animals survived well in captivity. Squids that had sustained skin trauma during capture and transport were probably most affected by the additional physiological stress of salinity and temperature shock. We believe that these squids probably accounted for most of the deaths within one to five days in captivity. 
TABLE I

Loligo plei: summary of capture, transport and transfer, and laboratory maintenance

\begin{tabular}{|c|c|c|c|c|c|c|c|c|}
\hline \multicolumn{3}{|c|}{ CAPTURE } & \multicolumn{6}{|c|}{$\begin{array}{l}\text { SHIPBOARD TRANSPORT (TP), LABORATORY TRANSFER } \\
\text { (TF), AND 1-DAY ACCLIMATION }\left(\mathrm{AC}_{1}\right)\end{array}$} \\
\hline $\begin{array}{l}\text { Obs. } \\
\text { No. }\end{array}$ & Date & $\begin{array}{c}\text { No. } \\
\text { Squids } \\
\text { Collected }\end{array}$ & $\begin{array}{l}\text { Transport } \\
\text { Container }\end{array}$ & $\begin{array}{c}\text { Trans- } \\
\text { port } \\
\text { Time } \\
\text { (h) }\end{array}$ & $\begin{array}{c}\text { Salinity } \\
\text { Change } \\
\text { (ppt) }\end{array}$ & $\begin{array}{c}\text { Temp. } \\
\text { Change } \\
\left({ }^{\circ} \mathrm{C}\right)\end{array}$ & $\begin{array}{l}\text { No. Dead } \\
\text { in TP, } \\
\text { TF, AC }\end{array}$ & $\begin{array}{c}\text { Percent } \\
\text { Mor- } \\
\text { tality }\end{array}$ \\
\hline 1 & 7 JUL 76 & 17 & $2001 \mathrm{VT}$ & 18 & $28-36$ & $32-29$ & 10 & $59 \%$ \\
\hline 2 & 8 AUG 76 & 21 & $\begin{array}{l}2001 \mathrm{VT} \\
3801 \mathrm{VT}\end{array}$ & 5 & $\begin{array}{l}33-32 \\
33-25\end{array}$ & $\begin{array}{l}30-27 \\
30-24\end{array}$ & 2 & $10 \%$ \\
\hline 3 & 22 AUG 76 & 18 & $2001 \mathrm{VT}$ & 4 & $\begin{array}{l}33-33 \\
33-28\end{array}$ & $\begin{array}{l}30-24 \\
30-24\end{array}$ & 6 & $33 \%$ \\
\hline 4 & 23 SEP 76 & 27 & $2001 \mathrm{VT}$ & 4 & $\begin{array}{l}30-35 \\
30-30\end{array}$ & $\begin{array}{l}27-23 \\
27-24\end{array}$ & 2 & $7 \%$ \\
\hline 5 & 2 OCT 76 & 15 & $2001 \mathrm{VT}$ & 24 & $29-32$ & $27-24$ & 11 & $73 \%$ \\
\hline 6 & 11 OCT 76 & 29 & $2001 \mathrm{VT}$ & 3 & $30-32$ & $24-22$ & 6 & $21 \%$ \\
\hline 7 & 1 NOV 76 & 80 & $2001 \mathrm{VT}$ & 3 & $35-32$ & $17-22$ & 77 & $96 \%$ \\
\hline 8 & 2 NOV 76 & 14 & $\begin{array}{l}2001 \mathrm{VT} \\
380 \mathrm{I} \text { VT }\end{array}$ & 3 & $36-32$ & $18-22$ & 7 & $50 \%$ \\
\hline 9 & 9 NOV 76 & 35 & $3801 \mathrm{VT}$ & 3 & $36-32$ & $21-22$ & 15 & $43 \%$ \\
\hline 10 & 4 DEC 76 & 28 & $3801 \mathrm{VT}$ & 24 & $35-32$ & $18-22$ & 25 & $89 \%$ \\
\hline 11 & 20 JAN 77 & 3 & 380 I HCT & 11 & $35-37$ & $17-21$ & 1 & $33 \%$ \\
\hline 12 & 17 MAR 77 & 1 & $2001 \mathrm{VT}$ & 4 & $26-35$ & $16-23$ & 0 & $0 \%$ \\
\hline 13 & 6 APR 77 & 12 & $3801 \mathrm{VT}$ & 8 & $27-36$ & $19-22$ & 4 & $33 \%$ \\
\hline 14 & 18 APR 77 & 14 & $3801 \mathrm{HCT}$ & 13 & $34-35$ & $22-24$ & 0 & $0 \%$ \\
\hline 15 & 25 MAY 77 & 11 & $3801 \mathrm{HCT}$ & 6 & $31-34$ & $26-21$ & 2 & $18 \%$ \\
\hline 16 & 25 MAY 77 & 22 & $3801 \mathrm{HCT}$ & 6 & $31-34$ & $26-21$ & 2 & $9 \%$ \\
\hline 17 & 25 MAY 77 & 58 & $3801 \mathrm{HCT}$ & $1-6$ & $30-37$ & $32-21$ & 10 & $17 \%$ \\
\hline 18 & 16 JUL 77 & 11 & $3801 \mathrm{HCT}$ & 3 & $34-34$ & $30-21$ & 0 & $0 \%$ \\
\hline 19 & 16 AUG 77 & 10 & $2001 \mathrm{VT}$ & 3 & $30-34$ & $32-22$ & 0 & $0 \%$ \\
\hline
\end{tabular}


TABLE I (Continued)

\begin{tabular}{|c|c|c|c|c|c|c|c|c|}
\hline \multirow{3}{*}{$\begin{array}{c}\text { Main- } \\
\text { tenance } \\
\text { Tank }\end{array}$} & \multirow{3}{*}{$\begin{array}{c}\text { Salinity } \\
\text { Range } \\
\text { (ppt) }\end{array}$} & \multicolumn{7}{|c|}{ LABORATORY MAINTENANCE AND SURVIVAL } \\
\hline & & \multirow{2}{*}{$\begin{array}{c}\text { Temp. } \\
\text { Range } \\
\left({ }^{\circ} \mathrm{C}\right)\end{array}$} & \multirow{2}{*}{$\begin{array}{l}\text { No. of } \\
\text { Squids } \\
\text { Main- } \\
\text { tained }\end{array}$} & \multirow[b]{2}{*}{ Sex } & \multicolumn{2}{|c|}{ 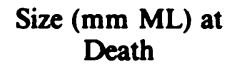 } & \multicolumn{2}{|c|}{$\begin{array}{c}\text { Laboratory } \\
\text { Survival (days) }\end{array}$} \\
\hline & & & & & $(\overline{\mathbf{x}})$ & (Range) & $(\overline{\mathbf{x}})$ & (Range) \\
\hline CT & $34-37$ & $24-30$ & $\begin{array}{l}3 \\
4\end{array}$ & $\begin{array}{l}\mathbf{M} \\
\mathbf{F}\end{array}$ & $\begin{array}{r}182 \\
92\end{array}$ & $\begin{array}{l}(165-205) \\
(82-98)\end{array}$ & $\begin{array}{l}17 \\
16\end{array}$ & $\begin{array}{l}(15-18) \\
(14-19)\end{array}$ \\
\hline $\begin{array}{l}\text { CT } \\
\text { CT }\end{array}$ & $\begin{array}{l}25-26 \\
32-33\end{array}$ & $\begin{array}{l}24-26 \\
23-27\end{array}$ & $\begin{array}{r}10 \\
9\end{array}$ & $\begin{array}{l}\mathbf{M} \\
\mathbf{F}\end{array}$ & $\begin{array}{r}129 \\
98\end{array}$ & $\begin{array}{r}(105-153) \\
(83-118)\end{array}$ & $\begin{array}{l}6 \\
9\end{array}$ & $\begin{array}{l}(2-11) \\
(3-18)\end{array}$ \\
\hline $\begin{array}{l}\text { CT } \\
\text { CT }\end{array}$ & $\begin{array}{l}33-35 \\
28-30\end{array}$ & $\begin{array}{l}23-25 \\
23-25\end{array}$ & $\begin{array}{l}6 \\
6\end{array}$ & $\begin{array}{l}\mathbf{M} \\
\mathbf{F}\end{array}$ & $\begin{array}{l}155 \\
102\end{array}$ & $\begin{array}{l}(140-175) \\
(100-107)\end{array}$ & $\begin{array}{l}17 \\
19\end{array}$ & $\begin{array}{l}(5-28) \\
(5-29)\end{array}$ \\
\hline CT & $30-35$ & $22-24$ & $\begin{array}{r}4 \\
5 \\
15\end{array}$ & $\begin{array}{l}\mathbf{M} \\
\mathbf{F} \\
\mathbf{J}\end{array}$ & $\begin{array}{r}103 \\
87 \\
41\end{array}$ & $\begin{array}{l}(71-138) \\
(74-102) \\
(31-50)\end{array}$ & $\begin{array}{r}4 \\
21 \\
3\end{array}$ & $\begin{array}{c}(2-6) \\
(15-29) \\
(2-6)\end{array}$ \\
\hline CT & $30-32$ & $22-24$ & 4 & $\mathbf{J}$ & 35 & $(30-41)$ & 3 & $(2-3)$ \\
\hline CT & $31-32$ & $22-24$ & $\begin{array}{r}11 \\
8 \\
3\end{array}$ & $\begin{array}{l}\mathbf{M} \\
\mathbf{F} \\
\mathbf{J}\end{array}$ & $\begin{array}{l}63 \\
59 \\
45\end{array}$ & $\begin{array}{l}(52-74) \\
(52-67) \\
(39-50)\end{array}$ & $\begin{array}{l}7 \\
3 \\
3\end{array}$ & $\begin{array}{l}(3-17) \\
(3-6) \\
(2-3)\end{array}$ \\
\hline CT & $32-33$ & $20-22$ & 3 & $\mathbf{J}$ & 41 & - & 16 & $(2-43)$ \\
\hline $\begin{array}{l}\text { CT } \\
\text { CT }\end{array}$ & $\begin{array}{l}33-34 \\
32-33\end{array}$ & $\begin{array}{l}21-23 \\
20-22\end{array}$ & $\begin{array}{l}3 \\
4\end{array}$ & $\begin{array}{l}\mathbf{M} \\
\mathbf{J}\end{array}$ & $\begin{array}{l}59 \\
42\end{array}$ & $\begin{array}{l}(55-63) \\
(34-48)\end{array}$ & $\begin{array}{l}4 \\
3\end{array}$ & $\begin{array}{l}(3-6) \\
(2-6)\end{array}$ \\
\hline $\begin{array}{l}\text { CT } \\
\text { CT }\end{array}$ & $\begin{array}{l}33-34 \\
32-33\end{array}$ & $\begin{array}{l}21-23 \\
20-22\end{array}$ & $\begin{array}{r}1 \\
19\end{array}$ & $\underset{\mathbf{J}}{\mathbf{M}}$ & $\begin{array}{l}53 \\
30\end{array}$ & $(2 \overline{1-45)}$ & $\begin{array}{l}3 \\
3\end{array}$ & $\frac{-}{(2-6)}$ \\
\hline CT & $35-36$ & $20-21$ & 3 & $\mathbf{J}$ & 37 & $(32-43)$ & 4 & $(3-6)$ \\
\hline CT & $35-37$ & $19-21$ & $\begin{array}{l}1 \\
1\end{array}$ & $\begin{array}{l}\mathbf{M} \\
\mathbf{J}\end{array}$ & $\begin{array}{l}85 \\
43\end{array}$ & - & $\begin{array}{l}84 \\
22\end{array}$ & - \\
\hline CT & $35-36$ & $21-23$ & 1 & $\mathbf{M}$ & 69 & - & 4 & - \\
\hline $\begin{array}{l}\text { CT } \\
\text { CT }\end{array}$ & $\begin{array}{l}35-35 \\
36-36\end{array}$ & $\begin{array}{l}23-23 \\
22-22\end{array}$ & $\begin{array}{l}2 \\
3\end{array}$ & $\begin{array}{l}\mathbf{M} \\
\mathbf{F}\end{array}$ & $\frac{95}{-}$ & $\overline{-}$ & $\begin{array}{l}3 \\
2\end{array}$ & $\begin{array}{l}(2-4) \\
(2-3)\end{array}$ \\
\hline $\begin{array}{l}\text { CT } \\
\text { RW }\end{array}$ & $\begin{array}{l}35-36 \\
34-37\end{array}$ & $\begin{array}{l}22-25 \\
16-23\end{array}$ & $\begin{array}{l}7 \\
5\end{array}$ & $\begin{array}{l}\mathbf{M} \\
\mathbf{F}\end{array}$ & $\frac{226}{-}$ & $\begin{array}{c}(204-243) \\
-\end{array}$ & $\begin{array}{l}33 \\
14\end{array}$ & $\begin{array}{r}(16-54) \\
(3-21)\end{array}$ \\
\hline CT & $34-37$ & $21-22$ & $\begin{array}{l}2 \\
5 \\
2\end{array}$ & $\begin{array}{l}\mathbf{M} \\
\mathbf{F} \\
\mathbf{J}\end{array}$ & $\begin{array}{r}140 \\
79 \\
43\end{array}$ & $\begin{array}{c}- \\
(54-95) \\
-\end{array}$ & $\begin{array}{r}16 \\
54 \\
4\end{array}$ & $\begin{array}{c}(8-24) \\
(14-52) \\
(2-6)\end{array}$ \\
\hline CT & $34-37$ & $20-21$ & $\begin{array}{r}3 \\
17\end{array}$ & $\begin{array}{l}\mathbf{M} \\
\mathbf{J}\end{array}$ & $\begin{array}{r}135 \\
40\end{array}$ & $\begin{array}{c}(105-160) \\
(38-44)\end{array}$ & $\begin{array}{r}56 \\
7\end{array}$ & $\begin{array}{r}(55-57) \\
(2-16)\end{array}$ \\
\hline RW & $31-37$ & $20-21$ & $\begin{array}{r}23 \\
21 \\
2\end{array}$ & $\begin{array}{l}\mathbf{M} \\
\mathbf{F} \\
\mathbf{J}\end{array}$ & $\begin{array}{r}118 \\
76 \\
48\end{array}$ & $\begin{array}{l}(64-223) \\
(51-123) \\
(47-50)\end{array}$ & $\begin{array}{l}17 \\
18 \\
25\end{array}$ & $\begin{array}{r}(2-49) \\
(3-45) \\
(22-28)\end{array}$ \\
\hline CT & $34-36$ & $20-21$ & $\begin{array}{l}4 \\
7\end{array}$ & $\begin{array}{l}\mathbf{M} \\
\mathbf{F}\end{array}$ & $\begin{array}{r}139 \\
83\end{array}$ & $\begin{array}{r}(110-164) \\
(65-101)\end{array}$ & $\begin{array}{l}10 \\
10\end{array}$ & $\begin{array}{l}(4-16) \\
(5-16)\end{array}$ \\
\hline CT & $34-35$ & $21-22$ & 10 & $\mathbf{J}$ & 19 & $(12-22)$ & 3 & $(2-4)$ \\
\hline
\end{tabular}




\begin{tabular}{|c|c|c|c|c|c|c|c|c|}
\hline \multicolumn{3}{|c|}{ CAPTURE } & \multicolumn{6}{|c|}{$\begin{array}{l}\text { SHIPBOARD TRANSPORT (TP), LABORATORY TRANSFER } \\
\text { (TF), AND 1-DAY ACCLIMATION }\left(\mathrm{AC}_{1}\right)\end{array}$} \\
\hline $\begin{array}{l}\text { Obs. } \\
\text { No. }\end{array}$ & Date & $\begin{array}{c}\text { No. } \\
\text { Squids } \\
\text { Collected }\end{array}$ & $\begin{array}{l}\text { Transport } \\
\text { Container } \\
\end{array}$ & $\begin{array}{c}\text { Trans- } \\
\text { port } \\
\text { Time } \\
\text { (h) }\end{array}$ & $\begin{array}{c}\text { Salinity } \\
\text { Change } \\
\text { (ppt) }\end{array}$ & $\begin{array}{c}\text { Temp. } \\
\text { Change } \\
\left({ }^{\circ} \mathrm{C}\right)\end{array}$ & $\begin{array}{c}\text { No. Dead } \\
\text { in } T P \text {, } \\
\text { TF, } A_{1}\end{array}$ & $\begin{array}{c}\text { Percent } \\
\text { Mor- } \\
\text { tality } \\
\end{array}$ \\
\hline 20 & 16 AUG 77 & 4 & $2001 \mathrm{VT}$ & 3 & $30-34$ & $32-22$ & 0 & $0 \%$ \\
\hline 21 & 18 AUG 77 & 7 & $3801 \mathrm{HCT}$ & $3-7$ & $28-32$ & $29-21$ & 4 & $57 \%$ \\
\hline 22 & 15 OCT 77 & 14 & $3801 \mathrm{HCT}$ & $12-36$ & $33-35$ & $27-21$ & 12 & $86 \%$ \\
\hline 23 & 30 OCT 77 & 13 & $3801 \mathrm{HCT}$ & 7 & $36-34$ & $26-21$ & 5 & $38 \%$ \\
\hline 24 & 10 MAR 78 & 3 & $3801 \mathrm{HCT}$ & 4 & $35-38$ & $14-20$ & 0 & $0 \%$ \\
\hline 25 & 26 APR 78 & 15 & $5501 \mathrm{HCT}$ & 3 & $35-35$ & $20-28$ & 3 & $20 \%$ \\
\hline 26 & 1 MAY 78 & 1 & $5501 \mathrm{HCT}$ & 3 & $34-36$ & $22-24$ & 0 & $0 \%$ \\
\hline 27 & 16 MAY 78 & 17 & $550 \perp \mathrm{HCT}$ & 6 & $38-32$ & $24-23$ & 0 & $0 \%$ \\
\hline 28 & 5 JUN 78 & 3 & $5501 \mathrm{HCT}$ & 15 & $34-36$ & $27-22$ & 3 & $100 \%$ \\
\hline 29 & 12 JUN 78 & 4 & $5501 \mathrm{HCT}$ & 2 & $25-31$ & $28-22$ & 1 & $25 \%$ \\
\hline 30 & 10 AUG 78 & 54 & $5501 \mathrm{HCT}$ & $3-5$ & $\begin{array}{l}25-32 \\
30-32\end{array}$ & $\begin{array}{l}29-21 \\
29-22\end{array}$ & 7 & $13 \%$ \\
\hline 31 & 20 MAY 82 & 75 & 10201 RHT & 6 & - & - & 26 & $35 \%$ \\
\hline 32 & 8 JUL 82 & 37 & 10201 RHT & 6 & - & - & 6 & $16 \%$ \\
\hline \multirow[t]{2}{*}{33} & 11 AUG 82 & 27 & 10201 RHT & 6 & - & - & 1 & $4 \%$ \\
\hline & & $\Sigma=700$ & & $\overline{\mathbf{x}}=7$ & $\begin{array}{c}\operatorname{Max} . \Delta= \\
+9,-8\end{array}$ & $\begin{array}{c}\operatorname{Max} . \Delta= \\
+8,-11\end{array}$ & & \\
\hline
\end{tabular}

Abbreviations: VT, vertical tank; CT, 2 m circular tank system; HCT, horizontal cylindrical tank; RHT, rectangular horizontal tank; RW, raceway tank; J, juvenile; * artificial sea water.

In all cases it was imperative not to overload the transport tanks or transfer bags, since this promoted wall contact, general excitement among the squids, and occasionally cannibalism. Long transport times and hot summer temperatures also increased mortality. Determination of the proper number of squids to be transported per tank is a behavioral consideration, not a physiological one, because water quality is good throughout the trip. The important considerations are the relative positioning of the squids to one another (this depends on the sizes of the squids) and to the tank 
TABLE I (Continued)

\begin{tabular}{|c|c|c|c|c|c|c|c|c|}
\hline \multirow{3}{*}{$\begin{array}{c}\text { Main- } \\
\text { tenance } \\
\text { Tank } \\
\end{array}$} & \multirow{3}{*}{$\begin{array}{l}\text { Salinity } \\
\text { Range } \\
\text { (ppt) }\end{array}$} & \multicolumn{7}{|c|}{ LABORATORY MAINTENANCE AND SURVIVAL } \\
\hline & & \multirow{2}{*}{$\begin{array}{l}\text { Temp. } \\
\text { Range } \\
\left({ }^{\circ} \mathrm{C}\right)\end{array}$} & \multirow{2}{*}{$\begin{array}{l}\text { No. of } \\
\text { Squids } \\
\text { Main- } \\
\text { tained }\end{array}$} & \multirow[b]{2}{*}{ Sex } & \multicolumn{2}{|c|}{$\begin{array}{c}\text { Size (mm ML) at } \\
\text { Death } \\
\end{array}$} & \multicolumn{2}{|c|}{$\begin{array}{c}\text { Laboratory } \\
\text { Survival (days) }\end{array}$} \\
\hline & & & & & $(\overline{\mathbf{x}})$ & (Range) & $(\overline{\mathbf{x}})$ & (Range) \\
\hline CT & 34-35 & $20-21$ & $\begin{array}{l}2 \\
2\end{array}$ & $\begin{array}{l}\mathbf{M} \\
\mathbf{F}\end{array}$ & $\begin{array}{r}106 \\
91\end{array}$ & $\begin{array}{l}(99-113) \\
(82-100)\end{array}$ & $\begin{array}{l}3 \\
3\end{array}$ & $\overline{-}$ \\
\hline RW & $32-35$ & $20-22$ & $\begin{array}{l}1 \\
2\end{array}$ & $\begin{array}{l}\mathbf{M} \\
\mathbf{F}\end{array}$ & $\overline{-}$ & $\overline{-}$ & $\begin{array}{r}23 \\
3\end{array}$ & $\overline{-}$ \\
\hline RW & $36-39$ & $18-22$ & $\begin{array}{l}1 \\
1\end{array}$ & $\begin{array}{l}\mathrm{M} \\
\mathrm{F}\end{array}$ & $\begin{array}{l}70 \\
67\end{array}$ & $\overline{-}$ & $\begin{array}{l}2 \\
2\end{array}$ & $\overline{-}$ \\
\hline CT & $34-36$ & $20-22$ & $\begin{array}{l}1 \\
6\end{array}$ & $\begin{array}{l}\mathbf{M} \\
\mathbf{J}\end{array}$ & $\begin{array}{l}53 \\
48\end{array}$ & $\overline{-}$ & $\begin{array}{l}2 \\
3\end{array}$ & $\overline{(2-7)}$ \\
\hline CT & 34-38 & $20-24$ & 3 & $\mathbf{F}$ & 64 & $(62-65)$ & 20 & $(5-31)$ \\
\hline CT & $35-37$ & $18-24$ & $\begin{array}{l}3 \\
8\end{array}$ & $\begin{array}{l}\mathbf{M} \\
\mathbf{F}\end{array}$ & $\begin{array}{l}123 \\
102\end{array}$ & $\begin{array}{r}(113-133) \\
(83-110)\end{array}$ & $\begin{array}{l}12 \\
14\end{array}$ & $\begin{array}{l}(7-19) \\
(6-20)\end{array}$ \\
\hline CT & $35-36$ & $24-24$ & 1 & $\mathbf{M}$ & 145 & - & 7 & - \\
\hline $\begin{array}{l}\text { CT } \\
\text { CT* }\end{array}$ & $\begin{array}{l}35-37 \\
30-32\end{array}$ & $\begin{array}{l}22-23 \\
20-22\end{array}$ & $\begin{array}{r}7 \\
10\end{array}$ & $\begin{array}{l}\mathbf{M} \\
\mathbf{F}\end{array}$ & $\begin{array}{l}210 \\
119\end{array}$ & $\begin{array}{l}(155-252) \\
(110-133)\end{array}$ & $\begin{array}{l}13 \\
14\end{array}$ & $\begin{array}{l}(8-22) \\
(6-27)\end{array}$ \\
\hline $\mathrm{CT}^{*}$ & $30-32$ & $21-24$ & $\begin{array}{l}1 \\
1 \\
1\end{array}$ & $\begin{array}{l}\mathbf{M} \\
\mathbf{F} \\
\mathbf{J}\end{array}$ & $\begin{array}{l}\overline{73} \\
-\end{array}$ & $\bar{z}$ & $\begin{array}{r}6 \\
23 \\
3\end{array}$ & $\overline{-}$ \\
\hline $\begin{array}{l}\mathrm{RW}^{*} \\
\mathrm{CT}\end{array}$ & $\begin{array}{l}32-35 \\
35-36\end{array}$ & $\begin{array}{l}21-24 \\
21-23\end{array}$ & $\begin{array}{l}26 \\
19\end{array}$ & $\begin{array}{l}\mathbf{M} \\
\mathbf{F}\end{array}$ & $\begin{array}{l}173 \\
109\end{array}$ & $\begin{array}{l}(112-232) \\
(107-139)\end{array}$ & $\begin{array}{l}10 \\
13\end{array}$ & $\begin{array}{l}(2-23) \\
(2-29)\end{array}$ \\
\hline $\begin{array}{l}\text { CT } \\
\text { CT* }\end{array}$ & $\begin{array}{l}34-34 \\
31-32\end{array}$ & $\begin{array}{l}22-23 \\
22-23\end{array}$ & $\begin{array}{r}12 \\
4\end{array}$ & $\begin{array}{l}\mathbf{M} \\
\mathbf{F}\end{array}$ & $\begin{array}{l}145 \\
112\end{array}$ & $\begin{array}{l}(88-180) \\
(92-131)\end{array}$ & $\begin{array}{l}5 \\
5\end{array}$ & $\begin{array}{l}(3-18) \\
(4-6)\end{array}$ \\
\hline $\mathbf{R} \mathbf{W}^{*}$ & - & - & $\begin{array}{l}26 \\
23\end{array}$ & $\begin{array}{l}\mathbf{M} \\
\mathbf{F}\end{array}$ & $\begin{array}{l}162 \\
106\end{array}$ & $\begin{array}{r}(115-200) \\
(86-124)\end{array}$ & $\begin{array}{l}7 \\
6\end{array}$ & $\begin{array}{l}(2-16) \\
(2-12)\end{array}$ \\
\hline $\mathbf{R} \mathbf{W}^{*}$ & - & - & 30 & $\mathrm{M}+\mathrm{F}+\mathrm{J}$ & - & - & 14 & $(3-36)$ \\
\hline \multirow[t]{2}{*}{$\mathbf{R W}^{*}$} & - & - & 25 & $\mathrm{M}+\mathrm{F}+\mathrm{J}$ & - & - & 13 & $(3-30)$ \\
\hline & $(25-39)$ & $(16-30)$ & $\Sigma=453$ & 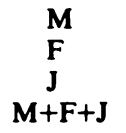 & $\begin{array}{r}145 \\
95 \\
35 \\
107\end{array}$ & $\begin{array}{l}(52-252) \\
(51-139) \\
(12-50) \\
(12-252)\end{array}$ & $\begin{array}{r}12 \\
13 \\
5 \\
11\end{array}$ & $\begin{array}{l}(2-84) \\
(2-52) \\
(2-43) \\
(2-84)\end{array}$ \\
\hline
\end{tabular}

configuration (especially the size of the horizontal dimensions of the tank) because the squid schools are generally dispersed horizontally, not vertically, in tanks. Based upon our experience in observing squids during transport and analyzing the reasons for mortality, we recommend ten full-sized Loligo spp. (150 to $250 \mathrm{~mm} \mathrm{ML}$ ) or 25 Lolliguncula brevis ( 40 to $80 \mathrm{~mm} \mathrm{ML)}$ per 550 l HCT tank. For the RHT tank we recommend 15 full-sized Loligo spp. per level (30 total). These are conservative estimates; under ideal conditions we have successfully transported greater numbers. 
TABLE II

Loligo pealei: summary of capture, transport and transfer, and laboratory maintenance

\begin{tabular}{|c|c|c|c|c|c|c|c|c|}
\hline \multicolumn{3}{|c|}{ CAPTURE } & \multicolumn{6}{|c|}{$\begin{array}{l}\text { SHIPBOARD TRANSPORT (TP), LABORATORY TRANSFER } \\
\text { (TF), AND 1-DAY ACCLIMATION }\left(\mathrm{AC}_{1}\right)\end{array}$} \\
\hline $\begin{array}{l}\text { Obs. } \\
\text { No. }\end{array}$ & Date & $\begin{array}{c}\text { No. } \\
\text { Squids } \\
\text { Collected }\end{array}$ & $\begin{array}{l}\text { Transport } \\
\text { Container }\end{array}$ & $\begin{array}{c}\text { Trans- } \\
\text { port } \\
\text { Time } \\
\text { (h) }\end{array}$ & $\begin{array}{c}\text { Salinity } \\
\text { Change } \\
\text { (ppt) }\end{array}$ & $\begin{array}{c}\text { Temp. } \\
\text { Change } \\
\left({ }^{\circ} \mathrm{C}\right)\end{array}$ & $\begin{array}{l}\text { No. Dead } \\
\text { in TP, } \\
\text { TF, } \mathbf{A C} \text {, }\end{array}$ & $\begin{array}{c}\text { Percent } \\
\text { Mor- } \\
\text { tality }\end{array}$ \\
\hline 1 & 20 FEB 77 & 7 & CT & - & $35-36$ & $16-16$ & 2 & $29 \%$ \\
\hline 2 & 18 AUG 77 & 1 & $3801 \mathrm{HCT}$ & 7 & $28-32$ & $29-21$ & 0 & $0 \%$ \\
\hline 3 & 15 OCT 77 & 8 & $3801 \mathrm{HCT}$ & 24 & $35-35$ & $27-21$ & 2 & $25 \%$ \\
\hline 4 & 19 OCT 77 & 2 & $3801 \mathrm{HCT}$ & 28 & $33-35$ & $27-21$ & 1 & $50 \%$ \\
\hline 5 & 30 OCT 77 & 1 & $3801 \mathrm{HCT}$ & 7 & $36-34$ & $26-21$ & 0 & $0 \%$ \\
\hline 6 & 23 MAY 78 & 1 & $5501 \mathrm{HCT}$ & 10 & $34-36$ & $25-22$ & 0 & $0 \%$ \\
\hline 7 & 2 JUN 78 & 7 & $5501 \mathrm{HCT}$ & 15 & $34-30$ & $27-22$ & 6 & $86 \%$ \\
\hline 8 & 23 JUN 78 & 10 & $5501 \mathrm{HCT}$ & 10 & $34-32$ & $27-22$ & 7 & $70 \%$ \\
\hline 9 & 25 OCT 78 & 6 & $5501 \mathrm{HCT}$ & 10 & $35-30$ & $26-21$ & 4 & $67 \%$ \\
\hline 10 & 27 APR 79 & 15 & $5501 \mathrm{HCT}$ & 48 & $36-36$ & $23-17$ & 8 & $53 \%$ \\
\hline 11 & 10 JUN 80 & 22 & $5501 \mathrm{HCT}$ & 10 & $33-32$ & $26-22$ & 一 & - \\
\hline 12 & 23 JUL 80 & 3 & $5501 \mathrm{HCT}$ & 10 & $35-33$ & $28-20$ & 1 & $33 \%$ \\
\hline 13 & 13 AUG 80 & 4 & $5501 \mathrm{HCT}$ & 10 & $36-36$ & $28-22$ & - & - \\
\hline 14 & 1 MAR 82 & 2 & 10201 RHT & 10 & $36-35$ & $18-21$ & - & - \\
\hline & & & & & $\begin{array}{l}\operatorname{Max} . \Delta= \\
+4,-5\end{array}$ & $\begin{array}{r}\operatorname{Max} . \Delta= \\
+3,-8\end{array}$ & & \\
\hline
\end{tabular}

Abbreviations: CT, 2 m circular tank system; HCT, horizontal cylindrical tank; RHT, rectangular horizontal tank; RW, raceway tank; * artificial sea water.

Sea water systems and water quality

Both systems provided adequate filtration capability as well as space for squids. As a rule of thumb, we determined that the $2 \mathrm{~m}$ circular tank system could maintain the following numbers of adult squids in a healthy state for several weeks: ten to 15 Loligo spp. (150 to $250 \mathrm{~mm} \mathrm{ML)} \mathrm{or} 25$ Lolliguncula brevis (40 to $80 \mathrm{~mm} \mathrm{ML)}$. Estimates for the 10,0001 raceway were determined to be: 50 Loligo spp. or 100 Lolliguncula brevis.

The tank systems were usually kept at the same approximate temperature and salinity as each species encountered in the wild at that month of the year, although fluctuations occurred. The reported ranges that squids are found in the northern Gulf of Mexico and were subjected to during our transport and maintenance work were: Loligo plei 13 to $32^{\circ} \mathrm{C}$ and 25 to $39 \mathrm{ppt}$; Loligo pealei 13 to $30^{\circ} \mathrm{C}$ and 28 to $39 \mathrm{ppt}$; Lolliguncula brevis 11 to $34^{\circ} \mathrm{C}$ and 18 to 39 ppt (Tables I, II, III; Rathjen et al., 1979; Hixon, 1980a; Hixon et al., 1980). 
SQUID MAINTENANCE, GROWTH, AND BEHAVIOR

TABle II (Continued)

\begin{tabular}{|c|c|c|c|c|c|c|c|c|}
\hline \multirow{3}{*}{$\begin{array}{c}\text { Main- } \\
\text { tenance } \\
\text { Tank }\end{array}$} & \multirow{3}{*}{$\begin{array}{c}\text { Salinity } \\
\text { Range } \\
\text { (ppt) }\end{array}$} & \multicolumn{7}{|c|}{ LABORATORY MAINTENANCE AND SURVIVAL } \\
\hline & & \multirow{2}{*}{$\begin{array}{c}\text { Temp. } \\
\text { Range } \\
\left({ }^{\circ} \mathrm{C}\right)\end{array}$} & \multirow{2}{*}{$\begin{array}{l}\text { No. of } \\
\text { Squids } \\
\text { Main- } \\
\text { tained }\end{array}$} & \multirow[b]{2}{*}{ Sex } & \multicolumn{2}{|c|}{$\begin{array}{c}\text { Size } \\
\text { Death }\end{array}$} & \multicolumn{2}{|c|}{$\begin{array}{c}\text { Laboratory } \\
\text { Survival (days) }\end{array}$} \\
\hline & & & & & $(\overline{\mathbf{x}})$ & (Range) & $(\overline{\mathbf{x}})$ & (Range) \\
\hline CT & 34-36 & $16-23$ & $\begin{array}{l}1 \\
4\end{array}$ & $\begin{array}{l}\mathbf{M} \\
\mathbf{F}\end{array}$ & $\begin{array}{l}213 \\
172\end{array}$ & $(15 \overline{4-200})$ & $\begin{array}{l}71 \\
23\end{array}$ & $\overline{(2-67)}$ \\
\hline RW & $32-35$ & $21-22$ & 1 & $\mathbf{F}$ & 167 & - & 21 & - \\
\hline $\mathbf{R W}$ & $36-39$ & $18-21$ & 6 & $\mathbf{M}$ & 202 & $(158-285)$ & 30 & $(15-41)$ \\
\hline RW & $36-39$ & $18-21$ & 1 & $\mathbf{M}$ & 183 & - & 5 & - \\
\hline CT & $34-36$ & $20-22$ & 1 & $\mathbf{F}$ & 121 & - & 30 & - \\
\hline CT & $32-37$ & $21-23$ & 1 & $\mathbf{M}$ & 109 & - & 53 & - \\
\hline $\mathrm{CT}^{*}$ & $31-32$ & $21-22$ & 1 & $\mathbf{F}$ & 174 & - & 25 & - \\
\hline $\mathrm{CT}^{*}$ & $31-32$ & $21-22$ & $\begin{array}{l}2 \\
1\end{array}$ & $\begin{array}{l}\mathbf{M} \\
\mathbf{F}\end{array}$ & $\begin{array}{l}152 \\
137\end{array}$ & $\begin{array}{c}(139-166) \\
-\end{array}$ & $\begin{array}{l}3 \\
2\end{array}$ & $\stackrel{(3-3)}{-}$ \\
\hline $\mathbf{R} \mathbf{W}^{*}$ & $30-34$ & $15-22$ & 4 & $\mathbf{F}$ & 163 & - & 36 & $(21-60)$ \\
\hline$C T$ & $36-36$ & $17-18$ & 6 & $\mathbf{M}+\mathbf{F}$ & - & - & 17 & $(3-35)$ \\
\hline $\mathbf{R} \mathbf{W}^{*}$ & $32-34$ & $21-22$ & 4 & $\mathbf{M}$ & 190 & $(140-153)$ & 44 & $(25-54)$ \\
\hline$C T$ & $33-36$ & $20-22$ & 2 & $\mathbf{M}$ & - & - & 27 & $(27-27)$ \\
\hline CT & $36-36$ & $21-22$ & 1 & $\mathbf{F}$ & 155 & - & 32 & - \\
\hline \multirow[t]{2}{*}{$\mathrm{CT}^{*}$} & $34-36$ & $20-22$ & 1 & $\mathbf{F}$ & 154 & - & 47 & - \\
\hline & $(30-39)$ & $(15-23)$ & $\Sigma=37$ & $\begin{array}{c}\mathbf{M} \\
\mathbf{F} \\
\mathbf{M}+\mathbf{F}\end{array}$ & $\begin{array}{l}184 \\
160 \\
174\end{array}$ & $\begin{array}{l}(109-285) \\
(121-200) \\
(109-285)\end{array}$ & $\begin{array}{l}31 \\
28 \\
28\end{array}$ & $\begin{array}{l}(3-71) \\
(2-71) \\
(2-71)\end{array}$ \\
\hline
\end{tabular}

Water quality remained high except in rare cases when high densities of animals (substantially greater than those mentioned above) were maintained for long periods of time. The Hach field test kits were useful only for gross estimates of nitrogenous buildup and for indicating increases, at which time detailed chemical tests were performed. The $\mathrm{pH}$ of each separate system was different, but the mean value for all experiments was 7.9, with a range of 7.7 to 8.5. Thirty-nine detailed water chemistry tests were performed among five CT systems during 1977 and 1978; these tests covered six different maintenance trials and all three squid species. The mean recorded level of total ammonia-nitrogen $\left(\mathrm{NH}_{4}-\mathrm{N}\right)$ from detailed chemical tests was $.103 \mathrm{mg} / \mathrm{l}$, with a range of .020 to $.161 \mathrm{mg} / \mathrm{l}(\mathrm{n}=12)$. Mean level of total nitrite-nitrogen $\left(\mathrm{NO}_{2}-\mathrm{N}\right)$ was $.003 \mathrm{mg} / \mathrm{l}$, with a range of .002 to $.007 \mathrm{mg} / \mathrm{l}(\mathrm{n}=12)$. Mean level of total nitratenitrogen $\left(\mathrm{NO}_{3}-\mathrm{N}\right)$ was $14.65 \mathrm{mg} / \mathrm{l}$, with a range of $9.98 \mathrm{mg} / \mathrm{l}$ to $20.73 \mathrm{mg} / \mathrm{l}(\mathrm{n}=15)$. In one separate observation, a male Loligo plei (124 mm ML) survived alone for 10 days in a 1501 aquarium that had approximate levels (from Hach test kits) of .185 $\mathrm{mg} / \mathrm{l}$ nitrite-nitrogen and $32.50 \mathrm{mg} / \mathrm{l}$ nitrate-nitrogen on Day 7 . Even assuming that 
TABLE III

Lolliguncula brevis: summary of capture, transport and transfer, and laboratory maintenance

\begin{tabular}{|c|c|c|c|c|c|c|c|c|}
\hline \multicolumn{3}{|c|}{ CAPTURE } & \multicolumn{6}{|c|}{$\begin{array}{l}\text { SHIPBOARD TRANSPORT (TP), LABORATORY TRANSFER } \\
\text { (TF), AND 1-DAY ACCLIMATION }\left(\mathrm{AC}_{1}\right) \\
\end{array}$} \\
\hline $\begin{array}{l}\text { Obs. } \\
\text { No. }\end{array}$ & Date & $\begin{array}{c}\text { No. } \\
\text { Squids } \\
\text { Collected }\end{array}$ & $\begin{array}{l}\text { Transport } \\
\text { Container }\end{array}$ & $\begin{array}{c}\text { Trans- } \\
\text { port } \\
\text { Time } \\
\text { (h) }\end{array}$ & $\begin{array}{c}\text { Salinity } \\
\text { Change } \\
\text { (ppt) }\end{array}$ & $\begin{array}{c}\text { Temp. } \\
\text { Change } \\
\left({ }^{\circ} \mathrm{C}\right)\end{array}$ & $\begin{array}{l}\text { No. Dead } \\
\text { in TP, } \\
\text { TF, AC, }\end{array}$ & $\begin{array}{c}\text { Percent } \\
\text { Mor- } \\
\text { tality }\end{array}$ \\
\hline 1 & 17 JUN 77 & - & $3801 \mathrm{HCT}$ & 1 & $29-21$ & $30-21$ & - & - \\
\hline 2 & 14 SEP 77 & 30 & $3801 \mathrm{HCT}$ & 1 & $23-30$ & $28-21$ & 23 & $77 \%$ \\
\hline 3 & 30 SEP 77 & 32 & $3801 \mathrm{HCT}$ & 1 & $\begin{array}{l}27-32 \\
27-26\end{array}$ & $\begin{array}{l}28-21 \\
28-21\end{array}$ & 5 & $16 \%$ \\
\hline 4 & 24 OCT 77 & 7 & $3801 \mathrm{HCT}$ & 1 & $24-36$ & $24-21$ & 2 & $29 \%$ \\
\hline 5 & 1 DEC 77 & 20 & $3801 \mathrm{HCT}$ & 1 & $24-36$ & $17-21$ & 7 & $35 \%$ \\
\hline 6 & 14 DEC 77 & 15 & $3801 \mathrm{HCT}$ & 1 & $25-26$ & $16-18$ & 6 & $40 \%$ \\
\hline 7 & 26 JAN 78 & 9 & $3801 \mathrm{HCT}$ & 8 & $34-35$ & $13-18$ & 1 & $11 \%$ \\
\hline 8 & 7 MAR 78 & 63 & $380 \mathrm{l} \mathrm{HCT}$ & 1 & $24-26$ & $13-20$ & 23 & $37 \%$ \\
\hline 9 & 20 JUN 78 & 7 & $550 \perp \mathrm{HCT}$ & 1 & $25-25$ & $28-21$ & 0 & $0 \%$ \\
\hline 10 & 29 JUN 78 & 38 & $2001 \mathrm{VT}$ & 1 & $24-32$ & $29-22$ & 5 & $13 \%$ \\
\hline 11 & 7 JUL 78 & - & $2001 \mathrm{VT}$ & 1 & $24-30$ & $29-22$ & - & - \\
\hline 12 & 24 JUL 78 & 13 & $2001 \mathrm{VT}$ & 1 & $26-30$ & $28-21$ & 1 & $8 \%$ \\
\hline 13 & 14 AUG 78 & 36 & $5501 \mathrm{HCT}$ & 1 & $32-24$ & $3 \cdot 1-23$ & - & - \\
\hline 14 & 24 OCT 78 & - & $5501 \mathrm{HCT}$ & 1 & $27-30$ & $23-21$ & - & - \\
\hline 15 & 22 JAN 79 & 12 & $5501 \mathrm{HCT}$ & 1 & $30-32$ & $11-22$ & 4 & $33 \%$ \\
\hline 16 & 5 JUL 79 & 42 & $5501 \mathrm{HCT}$ & 1 & $18-18$ & $29-21$ & 12 & $29 \%$ \\
\hline 17 & 27 FEB 80 & - & $5501 \mathrm{HCT}$ & 1 & $33-27$ & $19-20$ & - & - \\
\hline \multirow[t]{2}{*}{18} & 24 OCT 80 & - & - & - & - & - & - & - \\
\hline & & $\Sigma \sim 425$ & & $\overline{\mathbf{x}}=1$ & $\begin{array}{l}\max . \Delta= \\
+12,-8\end{array}$ & $\begin{array}{l}\max . \Delta= \\
+11,-9\end{array}$ & & \\
\hline
\end{tabular}

Abbreviations: VT, vertical tank; CT, 2 m circular tank system; HCT, horizontal cylindrical tank; RW, raceway tank; J, juvenile; * artificial sea water. 
TABLE III (Continued)

\begin{tabular}{|c|c|c|c|c|c|c|c|c|}
\hline \multirow{3}{*}{$\begin{array}{c}\text { Main- } \\
\text { tenance } \\
\text { Tank }\end{array}$} & \multirow{3}{*}{$\begin{array}{c}\text { Salinity } \\
\text { Range } \\
\text { (ppt) }\end{array}$} & \multicolumn{5}{|c|}{ LABORATORY MAINTENANCE AND SURVIVAL } & & \\
\hline & & \multirow{2}{*}{$\begin{array}{l}\text { Temp. } \\
\text { Range } \\
\left({ }^{\circ} \mathrm{C}\right)\end{array}$} & \multirow{2}{*}{$\begin{array}{l}\text { No. of } \\
\text { Squids } \\
\text { Main- } \\
\text { tained }\end{array}$} & \multirow[b]{2}{*}{ Sex } & \multicolumn{2}{|c|}{$\begin{array}{c}\text { Size (mm ML) at } \\
\text { Death }\end{array}$} & \multicolumn{2}{|c|}{$\begin{array}{c}\text { Laboratory } \\
\text { Survival (days) }\end{array}$} \\
\hline & & & & & $(\overline{\mathbf{x}})$ & (Range) & $(\overline{\mathbf{x}})$ & (Range) \\
\hline CT & $22-23$ & $21-22$ & $\begin{array}{r}8 \\
23 \\
10\end{array}$ & & $\begin{array}{l}56 \\
62 \\
34\end{array}$ & $\begin{array}{l}(51-62) \\
(44-85) \\
(27-40)\end{array}$ & $\begin{array}{r}13 \\
10 \\
4\end{array}$ & $\begin{array}{l}(2-58) \\
(2-59) \\
(2-6)\end{array}$ \\
\hline $\begin{array}{l}\text { CT } \\
C T\end{array}$ & $\begin{array}{l}23-25 \\
29-33\end{array}$ & $\begin{array}{l}20-22 \\
21-22\end{array}$ & $\begin{array}{l}3 \\
2 \\
2\end{array}$ & $\begin{array}{l}\mathbf{M} \\
\mathbf{F} \\
\mathbf{J}\end{array}$ & $\begin{array}{l}57 \\
82 \\
35\end{array}$ & $\begin{array}{c}(41-67) \\
(79-85) \\
-\end{array}$ & $\begin{array}{r}45 \\
40 \\
5\end{array}$ & $\begin{array}{c}(8-64) \\
(25-54) \\
-\end{array}$ \\
\hline $\begin{array}{l}\mathrm{RW} \\
\mathrm{CT} \\
\mathrm{CT}\end{array}$ & $\begin{array}{l}34-39 \\
26-36 \\
34-36\end{array}$ & $\begin{array}{l}18-22 \\
20-23 \\
20-21\end{array}$ & $\begin{array}{r}15 \\
11 \\
1\end{array}$ & $\begin{array}{l}\mathbf{M} \\
\mathbf{F} \\
\mathbf{J}\end{array}$ & $\begin{array}{l}51 \\
73 \\
34\end{array}$ & $\begin{array}{c}(45-57) \\
(63-80) \\
-\end{array}$ & $\begin{array}{l}23 \\
33 \\
39\end{array}$ & $\begin{array}{c}(2-68) \\
(12-55) \\
-\end{array}$ \\
\hline CT & $35-38$ & $20-23$ & $\begin{array}{l}2 \\
3\end{array}$ & $\begin{array}{l}\mathbf{M} \\
\mathbf{F}\end{array}$ & $\begin{array}{l}55 \\
70\end{array}$ & $\begin{array}{l}(53-56) \\
(67-72)\end{array}$ & $\begin{array}{l}21 \\
38\end{array}$ & $\begin{array}{r}(5-37) \\
(33-44)\end{array}$ \\
\hline CT & $34-36$ & $15-21$ & 7 & $\mathbf{M}+\mathbf{F}+\mathbf{J}$ & - & - & 29 & $(3-106)$ \\
\hline $\begin{array}{l}\mathrm{CT}^{*} \\
\mathrm{CT}\end{array}$ & $\begin{array}{l}24-27 \\
30-36\end{array}$ & $\begin{array}{l}15-24 \\
15-24\end{array}$ & $\begin{array}{l}7 \\
2\end{array}$ & $\begin{array}{l}\mathbf{M} \\
\mathbf{F}\end{array}$ & $\begin{array}{l}57 \\
65\end{array}$ & $\begin{array}{l}(53-60) \\
(59-72)\end{array}$ & $\begin{array}{l}76 \\
64\end{array}$ & $\begin{array}{l}(33-123) \\
(58-70)\end{array}$ \\
\hline $\mathrm{CT}^{*}$ & $28-34$ & $18-19$ & $\begin{array}{l}3 \\
5\end{array}$ & $\begin{array}{l}\mathbf{M} \\
\mathbf{F}\end{array}$ & $\begin{array}{l}56 \\
83\end{array}$ & - & $\begin{array}{l}22 \\
24\end{array}$ & $\begin{array}{l}(15-33) \\
(16-35)\end{array}$ \\
\hline CT & $25-26$ & $19-24$ & 40 & $\mathbf{M}+\mathbf{F}+\mathbf{J}$ & - & - & 20 & $(2-49)$ \\
\hline CT & $22-24$ & $21-22$ & $\begin{array}{l}3 \\
4\end{array}$ & $\begin{array}{l}\mathbf{M} \\
\mathbf{F}\end{array}$ & $\begin{array}{l}59 \\
71\end{array}$ & $\begin{array}{l}(58-60) \\
(62-76)\end{array}$ & $\begin{array}{l}46 \\
38\end{array}$ & $\begin{array}{l}(40-49) \\
(33-40)\end{array}$ \\
\hline $\mathrm{CT}^{*}$ & $32-34$ & $21-22$ & $\begin{array}{r}5 \\
7 \\
21\end{array}$ & $\begin{array}{l}\mathbf{M} \\
\mathbf{F} \\
\mathbf{J}\end{array}$ & $\begin{array}{l}43 \\
49 \\
36\end{array}$ & $\begin{array}{l}(41-50) \\
(42-69) \\
(29-40)\end{array}$ & $\begin{array}{l}6 \\
5 \\
3\end{array}$ & $\begin{array}{l}(4-11) \\
(3-12) \\
(2-5)\end{array}$ \\
\hline $\mathrm{CT}^{*}$ & $28-30$ & $20-21$ & 18 & $\mathbf{M}+\mathbf{F}+\mathbf{J}$ & - & - & 4 & $(2-8)$ \\
\hline $\mathrm{CT}^{*}$ & $30-30$ & $21-23$ & 12 & $\mathbf{M}+\mathbf{F}+\mathbf{J}$ & - & - & 8 & $(2-12)$ \\
\hline CT & $22-24$ & $22-24$ & $\begin{array}{l}9 \\
8\end{array}$ & $\begin{array}{l}\mathbf{M} \\
\mathbf{F}\end{array}$ & $\begin{array}{l}55 \\
71\end{array}$ & $\begin{array}{l}(50-59) \\
(52-99)\end{array}$ & $\begin{array}{l}6 \\
7\end{array}$ & $\begin{array}{l}(2-12) \\
(2-17)\end{array}$ \\
\hline CT & $30-32$ & $20-21$ & 28 & $\mathbf{M}+\mathbf{F}+\mathbf{J}$ & - & - & 13 & $(3-29)$ \\
\hline CT & $32-34$ & $20-22$ & 8 & $\mathbf{M}+\mathbf{F}+\mathbf{J}$ & - & - & 20 & $(3-27)$ \\
\hline CT & $18-18$ & $20-21$ & 30 & $\mathbf{M}+\mathbf{F}+\mathbf{J}$ & - & - & 12 & $(2-17)$ \\
\hline CT & $26-27$ & $19-22$ & $\begin{array}{l}3 \\
3\end{array}$ & $\begin{array}{l}\mathbf{M} \\
\mathbf{F}\end{array}$ & $\begin{array}{l}59 \\
68\end{array}$ & $\begin{array}{l}(56-60) \\
(66-69)\end{array}$ & $\begin{array}{l}72 \\
67\end{array}$ & $\begin{array}{l}(67-74) \\
(54-73)\end{array}$ \\
\hline CT & $34-36$ & $16-25$ & $\begin{array}{l}6 \\
2 \\
2\end{array}$ & $\begin{array}{l}\mathbf{M} \\
\mathbf{F} \\
\mathbf{J}\end{array}$ & $\begin{array}{l}52 \\
63 \\
35 \\
\end{array}$ & $\begin{array}{l}(48-56) \\
(60-66) \\
(32-38)\end{array}$ & $\begin{array}{r}68 \\
120 \\
41\end{array}$ & $\begin{array}{c}(51-115) \\
(115-125) \\
(28-53)\end{array}$ \\
\hline & $(18-39)$ & $(15-25)$ & $\Sigma=313$ & $\begin{array}{c}\mathbf{M} \\
\mathbf{F} \\
\mathbf{J} \\
\mathbf{M}+\mathbf{F}+\mathbf{J}\end{array}$ & $\begin{array}{l}54 \\
67 \\
36 \\
55\end{array}$ & $\begin{array}{l}(41-67) \\
(42-99) \\
(27-40) \\
(27-99)\end{array}$ & $\begin{array}{r}32 \\
25 \\
6 \\
19\end{array}$ & $\begin{array}{l}(2-123) \\
(2-125) \\
(2-53) \\
(2-125)\end{array}$ \\
\hline
\end{tabular}


there is a large source of error in the Hach test, these levels indicate that squids can tolerate concentrations at least somewhat higher than those recommended for marine animals by Spotte $(1979 \mathrm{a}, \mathrm{b})$ : ammonia $0.1 \mathrm{mg} / 1 \mathrm{NH}_{4}-\mathrm{N}$, nitrite $0.1 \mathrm{mg} / 1 \mathrm{NO}_{2}-\mathrm{N}$, and nitrate $20.0 \mathrm{mg} / \mathrm{l} \mathrm{NO}-\mathrm{N}$.

Circular tank systems used without algal tanks since 1978 have not shown substantially increased levels of inorganic nitrogen, nor has our recent raceway tank, which does not have a biodisc but depends mostly on the bacterial population in the filter bed of the adjoining CT system for biological filtration. The biodisc filter in our early raceway system (Fig. 3) increased the capacity for biological filtration. However, a drawback of the biodisc was the lack of control over the types of organisms that grew on it, some of them undesirable in a closed system. In our new raceway without the biodisc, it is likely that this extra filtration capacity is not needed with our currently used animal loads. Certainly our attention to cleanliness contributes to this result, since food remains are carefully removed daily and nearly all maintenance procedures recommended by Spotte (1979a, b) are followed. Slight shifts in pH and corresponding increases in nitrogen levels are dealt with quickly, usually by replacing a small percentage of the water volume with fresh, clean sea water.

Some other problems are noteworthy. In uncovered tanks in bright illumination, various algae and other unknown organisms grew on the tank walls and raceway bottoms (note the black growth on the raceway bottom in Fig. 3). Growth of these types of organisms is uncontrollable and some forms can be deleterious (e.g., some blue-green algae). Bacterial buildup, especially of potentially toxic Vibrio spp., can also occur on these substrates as well as on biodisc filters. Therefore, we occasionally clean the bottoms or keep tops on the tanks to reduce illumination.

No conspicuous differences were noted between the performance or longevity of natural sea water and artificial sea water. Some CT systems have been in continuous use for as long as two years with no major alterations, aside from periodic ten percent water changes, occasional addition of trace metals, and occasional gentle stirring of the filter bed to siphon off excess detritus buildup that can clog the filter bed and reduce denitrification by bacteria.

\section{General aspects of behavior}

Healthy, calm squids of these three species do not bang haphazardly into aquarium walls. Squid vision is keen and they can quickly and deftly maneuver without hitting walls or other objects. Their behavior changes, however, if: (1) they are placed in small tanks, (2) they have incurred significant skin or fin damage, (3) they are engaged in intense intraspecific aggression, or (4) they are not fed. It is important to recognize normal versus altered behavior because it is possible to preclude or reduce circumstances that promote altered behavior, which leads to decreased survival in captivity.

Loliginid squids are social, schooling, inquisitive creatures that actively react to everything in their environment. Nearly all aspects of squid behavior are mediated through expression of the chromatophore system, as well as particular postures and movements; collectively these are referred to as body patterns (Hanlon, 1982).

Loligo plei (Fig. 4) has the widest range of body patterns and the most complex behavior. To date, 16 chromatic and six postural components of body patterning have been described and associated with specific behavior (Hanlon, 1982, and in prep.). Males grow larger than females, they are far more aggressive, and they possess seven male-only chromatic components that are used in an intraspecific aggressive context and are inextricably connected with courting and mating behavior (Hanlon, 1981, 1982). Males establish and maintain a rank order based upon size and ag- 

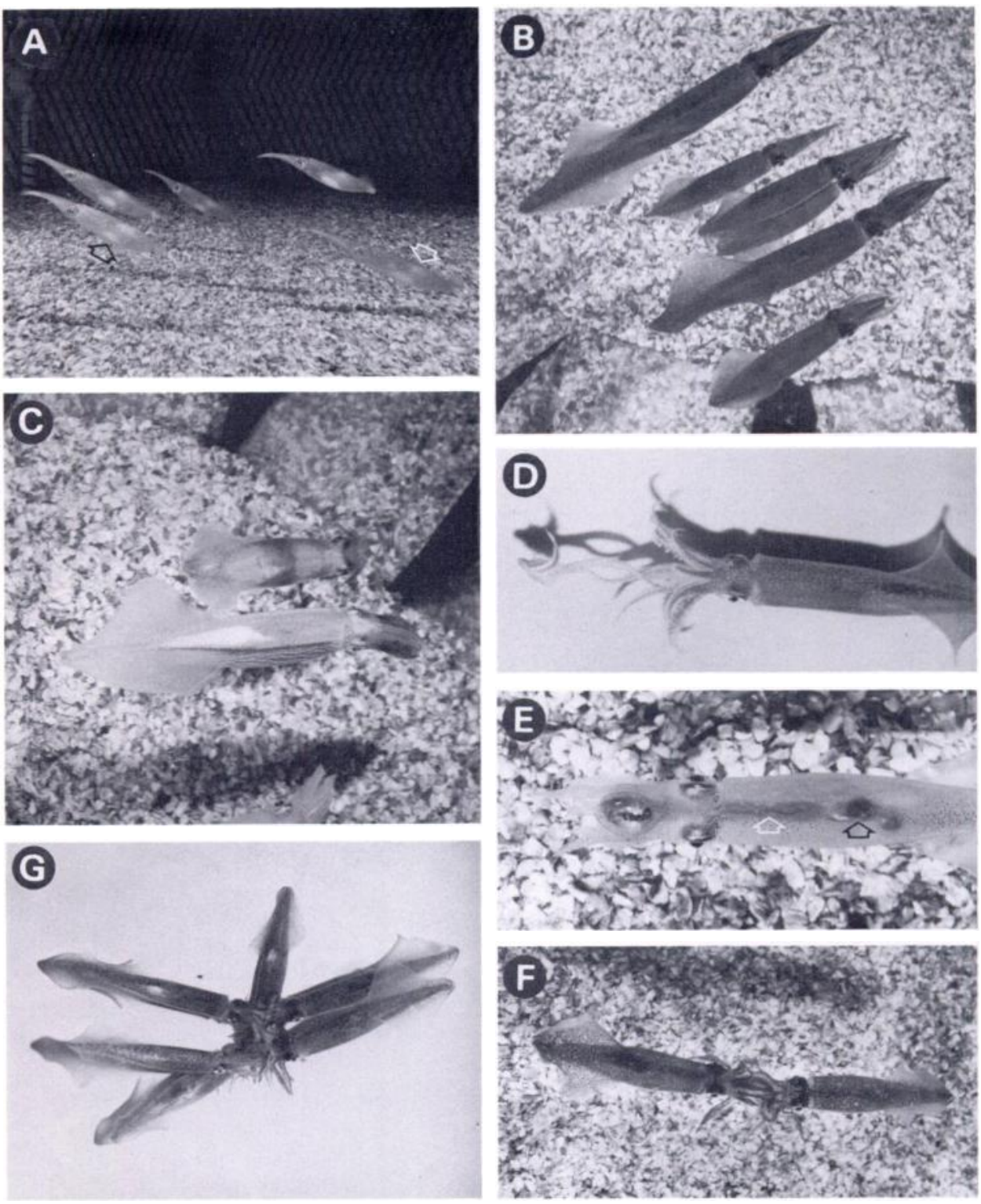

Figure 4. Loligo plei. A. Five females ( 74 to $102 \mathrm{~mm} \mathrm{ML}$ ) schooling loosely during Observation 4 (see Table I). The Clear pattern indicates calmness. Note the well-developed ovaries (white arrow) and nidamental glands (black arrow) characteristic of very mature females. In color, the red accessory nidamental glands are also visible. B. Three males ( 165 to $205 \mathrm{~mm} \mathrm{ML}$ ) and four females ( 82 to $98 \mathrm{~mm} \mathrm{ML}$ ) from Observation 1, schooling tightly in a CT system. The All Dark pattern indicates that the squids are alarmed. C. Intraspecific aggression and mate pairing during Observation 1. The large dominant male (center, 205 $\mathrm{mm} \mathrm{ML}$ ) is performing a "lateral display" towards the male on its right (bottom, $175 \mathrm{~mm} \mathrm{ML}$ ) in order to keep his female mate ( $93 \mathrm{~mm} \mathrm{ML}$ in Ring pattern) segregated from the school. Mating and egg laying occurred the same day. D. A small live fish is seized by the extended tentacles of a male squid, $174 \mathrm{~mm}$ ML. Note the buckling of the tentacles (see Kier, 1982). E. A female (110 mm ML) from Observation 27 eating a small fish. Note that the fish is held vertically and that the viscera are being eaten first. The digestive gland is swollen and reddish (white arrow) and the stomach is approximately $1 / 3$ full (black arrow). F. A male (left, $113 \mathrm{~mm} \mathrm{ML}$ ) and female (110 mm ML) from Observation 25 in a tug-of-war over a fairly large fish. G. Cannibalism. Six males (approx. $220 \mathrm{~mm} \mathrm{ML}$ ) jointly eating another male that had been moribund prior to cannibalization. 
gressiveness. They accomplish this mainly through visual signalling, in particular a "lateral display" in which the males position themselves in parallel and then unilaterally flash flame-like streaks of chromatophores on the lateral mantle towards one another (Fig. 4C). Up to five additional chromatic components may be expressed in this display, depending upon its intensity. In some cases the squids may also engage in "fin beating" while parallel to one another, and in extremely rare cases the dominant (and usually larger) squid may execute a forward attack and grasp or bite the other squid. In contrast, females are generally passive and docile in the laboratory (Fig. 4A, B, C) and they seldom engage in aggressive behavior except occasionally during the pursuit of prey (Fig. 4F). Mating and egg laying are common in captivity and can be artificially stimulated by placing egg strands or a facsimile in the tank in the manner described for Loligo pealei by Arnold (1962). Feeding and growth in captivity are good, with cannibalism (Fig. 4G) occurring rarely. L. plei has delicate skin and is more vulnerable to skin abrasion than the other two species (Fig. 7).

Loligo pealei (Fig. 5) has the second widest range of body patterns and its behavior


FIGURE 5. Loligo pealei. A. Intraspecific compatibility is obvious in this school of three Loligo pealei (two males, one female at far left) and two Loligo plei males (arrows). All squids are approximately 220 $\mathrm{mm}$ ML. Note the wall pattern and how squids stay near the middle of the tank. B. Female (174 mm ML) in a Ring pattern while bottom sitting. This is a normal posture for this species. C. Female $(180 \mathrm{~mm} \mathrm{ML})$ actively securing an egg strand into the substrate. Note the egg strand on the left; also the bold stripes on the wall. 
is similar in complexity to $L$. plei. Approximately 12 chromatic and four postural components and their associated behavior are recognizable in this species. Males and females are similar in size (Hixon et al., 1981) and grow larger than L. plei. Males and females both display intraspecific aggression. Males are slightly more aggressive and they also establish a rank order based upon size and aggressiveness (similar to that reported by Arnold, 1962), but they do not show any obvious male-only or female-only chromatic components. Mating and egg laying are common in captivity (Fig. 5C) and can be easily stimulated (Arnold, 1962). Feeding and growth are good, as in $L$. plei, but cannibalism by large males is more common. The skin is nearly as subject to injury as in L. plei (Fig. 7).

Loligo pealei commonly sits on the bottom (Fig. 5B). This is a normal posture, exclusive to this species, that is assumed for long periods of time on sand or gravel substrates. Bottom sitting is conducive to laboratory survival because it conserves energy (compared to constant swimming), it maintains calmness among the tank animals, and it minimizes contact with the tank walls.

Loligo plei and Loligo pealei are very similar morphometrically and they are difficult to distinguish visually, especially when they are smaller than $100 \mathrm{~mm} \mathrm{ML}$. They can, however, be distinguished by their specific chromatic components (particularly L. plei males) and their behavior, and this is useful for the identification and segregation of animals in the laboratory.

Lolliguncula brevis (Fig. 6) is distinctly different from the two species of Loligo. Aside from being smaller, its behavior is less complex and only seven chromatic and four postural components of body patterning have been noted thus far. A common threat posture is illustrated in Figure 6D. Females grow markedly larger than males (Dragovich and Kelly, 1962; Hixon, 1980a). Little intraspecific aggression has been observed and there has been no evidence of rank ordering among males. Mating has been seen fairly often, and large females are often seen with conspicuous white patches of spermatophores attached to a pad on the inside of the mantle on the left side (Fig. 6A); however, egg laying in captivity is rare. Efforts to stimulate egg laying with egg strands were negative, but occasionally a temperature increase resulted in egg laying. Feeding and growth in captivity are very good (Fig. 6B, C). This species is less vulnerable to fin and skin damage than Loligo spp. For these reasons, males and females may be kept in the same tank at higher densities (Fig. 6A) than Loligo spp. and for longer periods of time.

There is some interspecific compatibility among the three species. On several occasions mid- to large-sized Loligo pealei and Loligo plei have been kept in CT systems for up to 15 days with no noticeable problems. It was important that these animals were all of a similar size (about $200 \mathrm{~mm} \mathrm{ML}$ ) and were put into this tank at the same time. They schooled together in a seemingly random arrangement, i.e., individuals were found in different parts of the school at different times (Fig. 5A). During another observation, nine Loligo plei were put into a tank that held a mating pair and eggs of Loligo pealei. The male Loligo pealei continuously displayed towards and attacked the $L$. plei, which had to be removed within one day. It was difficult to tell whether this was territorial defense of the eggs, the female, or the tank, or simply the usual aggression shown by large males to define the rank order. Lolliguncula brevis is compatible with both species of Loligo if all animals are of the same size; they even school together with little interaction. But if the Loligo are larger they will display towards the Lolliguncula brevis, which in turn will often display and attack as well. It is characteristic of Loligo plei and Loligo pealei to cannibalize smaller squids or weakened squids such as those with impaired swimming due to skin damage or the effects of anaesthetic agents. Cannibalism by Lolliguncula brevis has been 

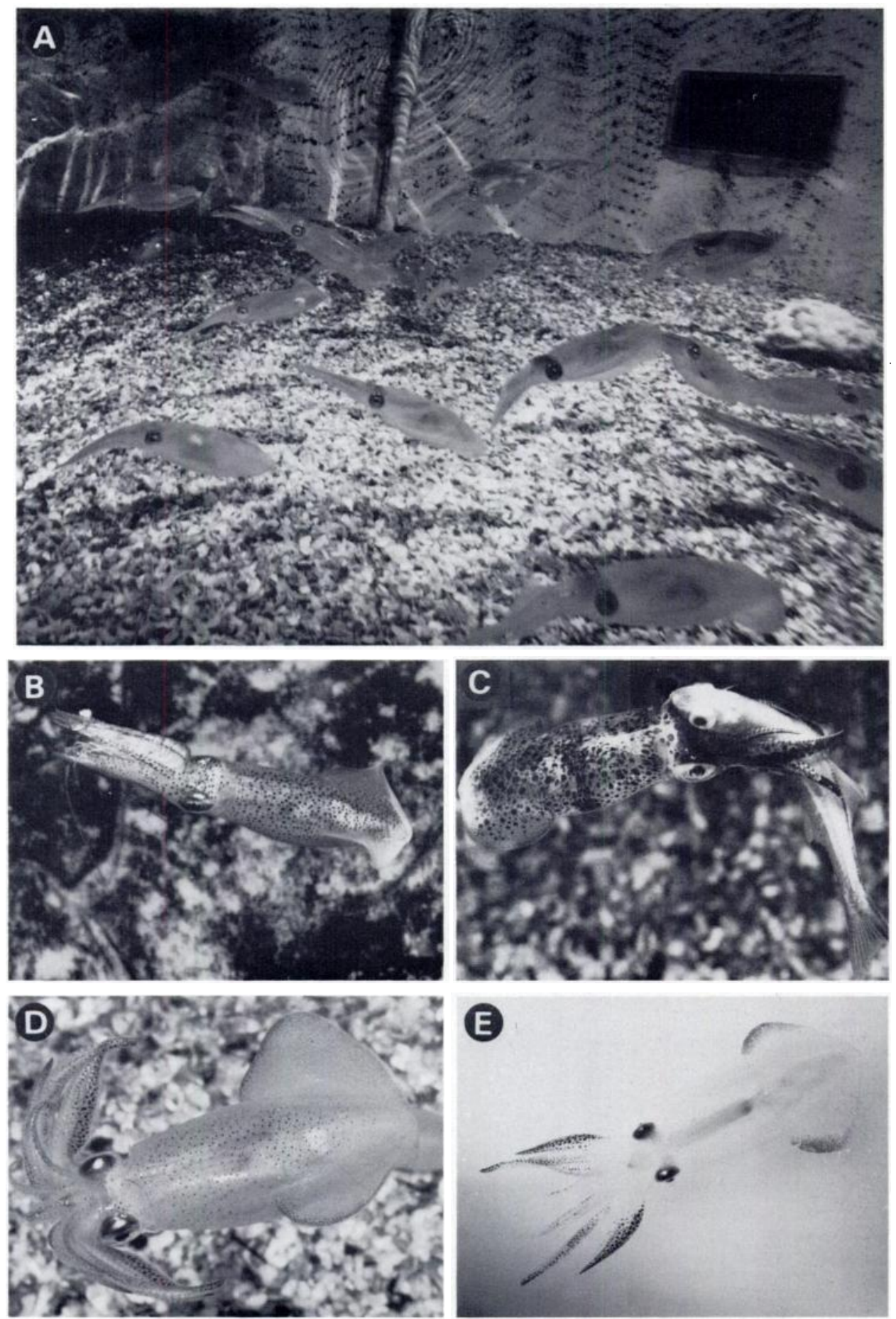

FIGURE 6. Lolliguncula brevis. A. Twenty-three squids in a CT system. Note the white patch of spermatophores inside the mantle on the female in the left foreground. B. Small squid feeding on a penaeid 

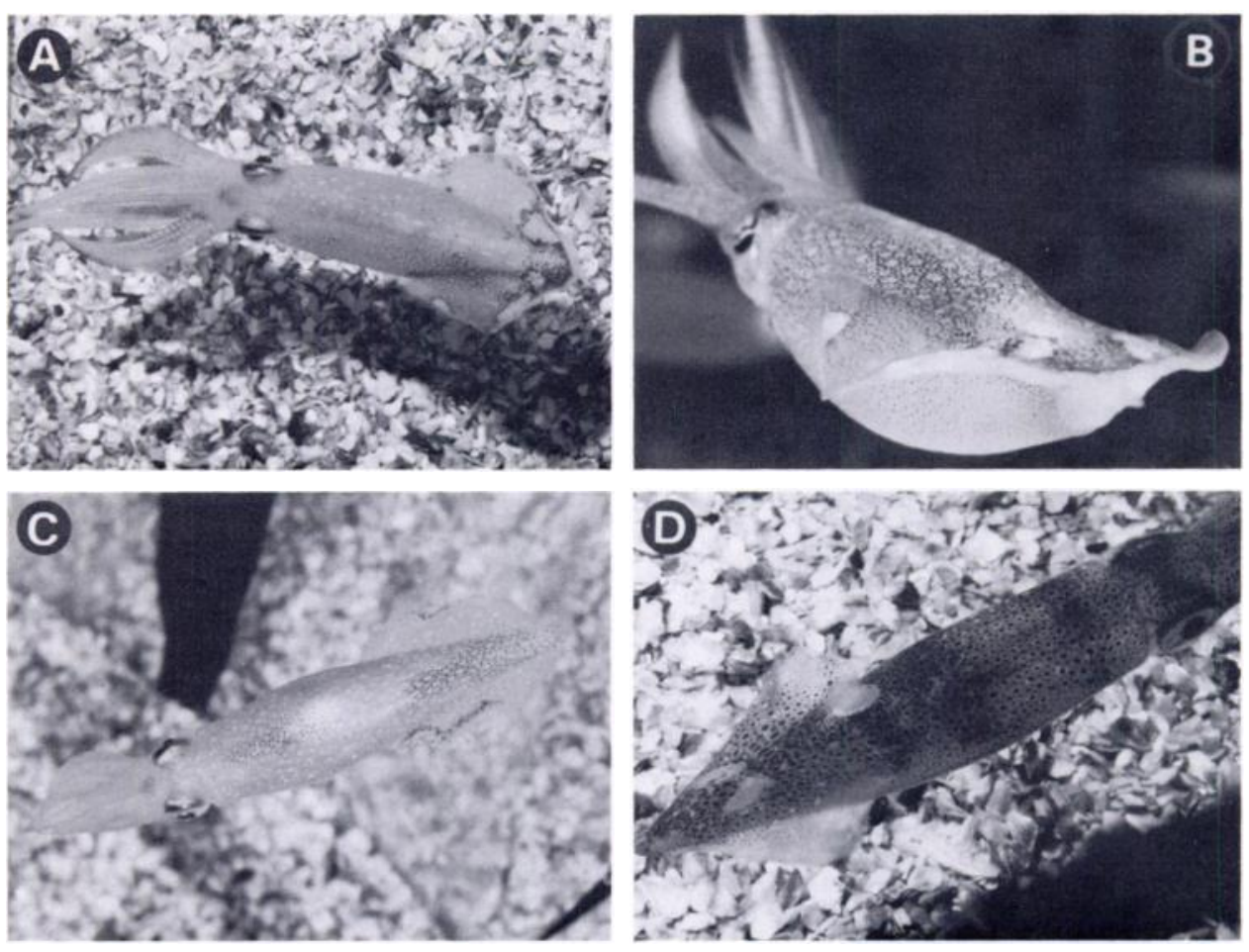

FIGURE 7. Fin damage. A. Loligo pealei with fairly severe fin damage incurred initially from transport. B. The same squid as in (A) showing the amount of damage on the posterior fin and the ventral mantle that resulted from hitting the transport tank walls. C. Loligo plei female $(82 \mathrm{~mm} \mathrm{ML)} \mathrm{from} \mathrm{Observation}$ 1 with moderate fin damage. Note the expanded chromatophores that are usually present around the periphery of damage. D. Loligo plei female $(87 \mathrm{~mm} \mathrm{ML})$ with several round patches of damage; these are less lethal than damage to the periphery of the fin.

observed only on one rare occasion. In summary, under ideal circumstances there is interspecific compatibility among the three species, but when a size difference exists the larger individual usually dominates.

As the three species were exposed to salinity shock when first brought to the laboratory, their reactions were immediate. The first manifestation of stress was the curling of the extreme distal portions of the eight arms. The squids also showed sluggish hovering and swimming movements and usually did not school or feed immediately. In extreme cases, some squids would go to the bottom and sit, a posture very uncommon to Lolliguncula brevis and Loligo plei, but not unusual for Loligo pealei. Normal behavior usually resumed within one hour or less.

Field behavior was also species specific. Loligo plei was nearly always found in moderate- to large-sized schools both during the day and night. Around night light stations, the schools usually stayed deep and would characteristically rise en masse to the surface under the light, then quickly dive. They preferred the periphery of

shrimp nearly as long as the squids' mantle length. C. Small squid from Observation 16 (Table III) eating a very large silverside, Menidia beryllina. D. A female swimming in a typical threat posture three days after brain surgery, in which the vertical lobe was cut. E. Narcotized squid with the characteristic chromatophore pattern that is usually produced while the squid is under anaesthesia. 
light. On occasion, one or several squids would leave the school to feed. Only rarely would the schools swarm for any period of time right at the surface under the lights in the manner described for the California market squid Loligo opalescens (Kato and Hardwick, 1975). Individual schools would seldom stay around the night light station very long, but other schools would appear later, indicating that squids were moving and actively foraging. Schools became closer knit and usually left the night light stations upon the appearance of schools of scad or jacks (Family Caranjidae), mackeral, or sharks. Schools always were comprised of squids of similar size, an observation corroborated by laboratory results that showed squid schools being incompatible when size disparities were present. In some cases it appeared that schools were not only size specific but sex specific as well [the white testis of mature males is highly conspicuous in live squids and is even used in signalling (Hanlon, 1982)]. At Grand Cayman Island, B.W.I., large schools could be attracted to night lights set on sandy patches between coral reefs at $10 \mathrm{~m}$. These schools were never seen near reefs during the day, at which time they presumably moved to deeper water. Off Eleuthera Island, Bahamas, we have observed one small Loligo (probably plei) swimming on three consecutive days with a school of 12 Sepioteuthis sepioidea. Moynihan and Rodaniche (1982) observed this association frequently in Panama.

Loligo pealei behaved quite differently from Loligo plei in the field. Our only observations were at deep-water night light stations, and in most cases large adults were seen singly or in pairs. No tight, well-formed adult schools were ever observed at night light stations, although on some nights enough individuals would arrive at the lights over a period of time to form a loose aggregate of squids. In a few cases we could identify the pairs as male-female, presumably a mating pair. In contrast to adults, young juveniles were often seen in large schools under the lights, indicating that $L$. pealei becomes more solitary at night as it becomes larger. Lolliguncula brevis observations are scarce due to the turbid waters in which it lives.

\section{Feeding}

All three squid species fed readily on a variety of live fishes and shrimps (Figs. $4,5,6$ ). The feeding response of adult squids in good physical condition has been excellent, with detection, pursuit, and capture of prey usually taking five seconds or less. Some squids fed within ten minutes of their release into the tank and nearly all fed within the first day of confinement. The feeding behavior of healthy squids is predictable and provides an indication of the animals' overall condition. Damage to the delicate fins seriously impaired the squids' ability to deftly maneuver for prey capture. Squids actively participating in intraspecific aggressive behavior often did not feed well; conversely, feeding occasionally led to aggressive behavior when two or three squids would capture the same prey organism and a vigorous tug-of-war would result (Fig. 4F).

The response of juvenile squids to the presence of food was usually slower, with feeding sometimes not beginning for a day or two and remaining sporadic thereafter. One probable cause for this was their greater susceptibility to fin damage during capture and transport to the laboratory. Another cause, in some cases, was the presence of large conspecifics that were aggressive.

Movement on the part of the prey provided an essential visual stimulus to the squids. Fishes or shrimps that made it to the bottom of the tank without detection would go uneaten for hours if they remained motionless. In the CT systems, the oyster shell substrate and the painted walls provided partial concealment because of the similarity in coloration between the substrate and prey organisms. Palaemonid 
shrimps blended in especially well, and even when there were hundreds of them in the tank, only those that moved quickly or swam into the water column were attacked and eaten. In the raceway system, squids had little difficulty in sighting and capturing prey against the pale interior of new or cleaned systems, but on algae-covered bottoms (e.g., Fig. 3) small shrimps were difficult to detect. Normally, all squids ate daily at each of the two or three times food was dropped into the tanks, even though food was usually in the tanks at all times. During growth experiments, Loligo plei consumed 10 to 18 percent of its body weight in food per day (see Growth section).

Small fishes (less than about $25 \mathrm{~mm}$ long) were usually eaten entirely. Larger fishes were captured with the two long tentacles (Fig. 4D) and were bitten several times through the vertebrae just behind the head, after which the viscera were eaten (Fig. 4E) and all the meat on either side of the skeleton was cleanly stripped away. Shrimps were eaten completely except for some of the head and the thin exoskeleton. Hungry squids sometimes took prey nearly as long as their own mantle length (Fig. $6 \mathrm{~B}, \mathrm{C})$.

It was not possible to detect any clear-cut diet preferences for different species or different growth stages. The younger stages of all three species seemed to prefer crustaceans, and the larger animals generally preferred fishes, but many individual and collective exceptions to this statement occurred. Cannibalism occurred rarely (see Behavior section). Growth rates were equally high on shrimp-only, fish-only, and mixed diets, and our conclusion is that estuarine food organisms are suitable for maintaining and growing loliginid squids.

Field and laboratory observations both confirmed that squids of all sizes eat prey organisms of a wide size range. Underwater observations during night lighting stations off Texas and Grand Cayman verified that adult Loligo plei commonly fed on very small plankton in the vicinity of the night light. The squids always seemed to be very selective about these planktonic organisms, for they would carefully orient towards, follow, and seize specific organisms even when great masses of plankton were present. Conversely, squids at the same station would inspect and sometimes attack squid jigs up to $70 \mathrm{~mm}$ long, objects that were many orders of magnitude larger than the planktonic organisms they had seized minutes before. Laboratory observations corroborated this behavior. We commonly saw adult squids follow and inspect small bubbles that were only several millimeters in diameter.

\section{Survival}

Loligo plei ranging in size from 12 to $252 \mathrm{~mm}$ ML (mean ML 107, S $\overline{\mathbf{x}}=3.0$ ) were maintained in 33 laboratory observations (Table I). The mean survival time for 455 squids was 11 days $(S \bar{x}=0.5$, median $=7$ days, Fig. 8$)$. The longest-lived male ( $85 \mathrm{~mm} \mathrm{ML}$ ) survived 84 days, and two females (89 and $95 \mathrm{~mm} \mathrm{ML}$ ) survived for a maximum of 52 days. There were no significant differences in survival time between males $(n=149$, median survival time 7 days) and females $(n=132$, median survival time 10 days). In contrast, the survival times of 81 juveniles (less than $50 \mathrm{~mm} \mathrm{ML}$ ) were low. Median survival was three days, which was significantly $(P<.001)$ lower than both males and females.

Loligo pealei showed best overall survival in our tank systems (Table II). Squids ( $\mathrm{n}=37$ ) ranging in size from 109 to $285 \mathrm{~mm}$ ML (mean $173 \mathrm{~mm} \mathrm{ML}, \mathrm{S} \overline{\mathbf{x}}=7.7$ ) had a mean survival time of 28 days $(S \bar{x}=3.1$, median $=27$ days, Fig. 8). The maximum survival time was 71 days for a male measuring $213 \mathrm{~mm} \mathrm{ML}$. The longestlived female survived 67 days and measured $200 \mathrm{~mm}$ ML. There were no statistically significant differences in survival by sex; 17 males had a median survival time of 28 


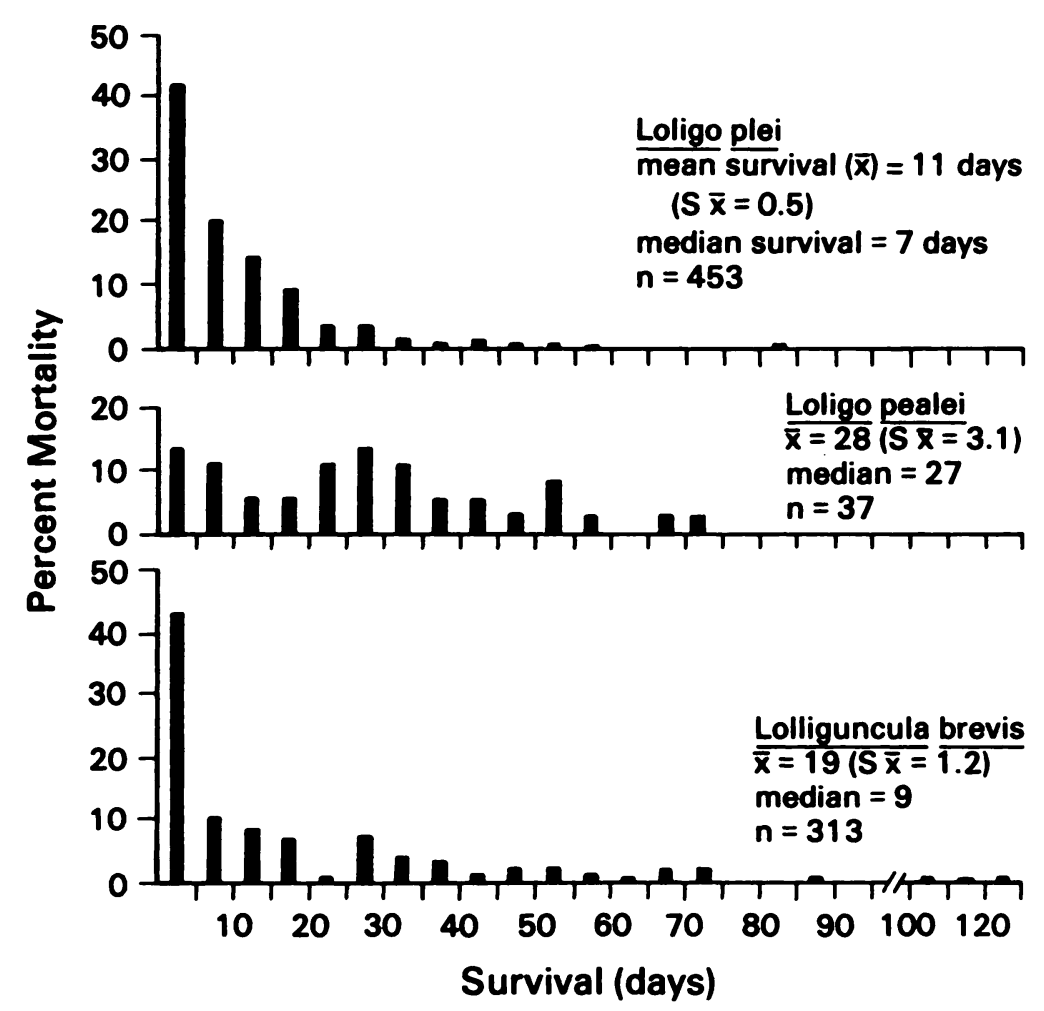

FIGURE 8. Survival summary of all squids in recirculating circular tanks and raceways.

days, and 14 females had a median survival time of 27.5 days. No small-sized juveniles of this species were maintained during the 14 laboratory observations.

A total of 313 Lolliguncula brevis ranging in size from 27 to $99 \mathrm{~mm}$ ML (mean ML $55 \mathrm{~mm}, \mathrm{~S} \overline{\mathrm{x}}=1.2$ ) were maintained during the course of 18 laboratory observations (Table III). The mean survival time for all squids was 19 days $(S \bar{x}=1.3$, median $=9$ days, Fig. 8). Some squids commonly survived much longer; the longest-lived male (60 mm ML) survived 123 days, and the longest-lived female (also $60 \mathrm{~mm} \mathrm{ML}$ ) survived for 125 days. There were no statistically significant differences in survival times between 63 males (median survival 19 days) and 74 females (median survival 14 days). The median survival time of 36 juveniles (less than $40 \mathrm{~mm} \mathrm{ML}$ ) was only 3.5 days; this was significantly lower $(P<.001)$ than that of males and females.

There were statistically significant differences in laboratory survival among the three species. Loligo pealei survived in the laboratory tanks significantly longer ( $P$ $<.001)$ than both Loligo plei or Lolliguncula brevis, and Lolliguncula brevis survived significantly longer $(.001<P<.01)$ than $L$. plei.

It should be pointed out that the mean survival times for all three species shown above are conservative figures. In these estimates all squids alive in the laboratory after one day of acclimation were included, regardless of their size or physical condition after capture and transport. The inclusion in the calculations of the short-lived juveniles and sexually-mature adults near the end of their life cycle also reduced the overall mean and median values. If the effects of these factors are reduced by computing laboratory survival using only squids that lived beyond five days, instead of one, then the mean survival time for each species increases substantially. The mean survival 
of Loligo plei becomes 15 days $(S \overline{\mathrm{x}}=0.7)$ compared to 11 days. Likewise, mean survival for Loligo pealei increases to 31 days $(S \bar{x}=3.1)$ from 28 days, and Lolliguncula brevis increases to 29 days $(S \bar{x}=1.7)$ from only 19 days.

These higher figures probably represent a more realistic approximation of how long squids survive in captivity, because they do not include squids that incurred extensive skin damage during capture, transport, and transfer. In effect, one can then begin to define the limitations to survival among these squids in terms of aspects of behavior and maturation that take place in the laboratory tank system. These are explained below (Principal causes of mortality).

\section{Growth}

Laboratory observations on growth were obtained from three male and three female Loligo plei and from seven male and one female Loligo pealei; temperatures ranged from 18 to $23^{\circ} \mathrm{C}$ (Table IV). The results suggest that adult males of both species of Loligo are capable of growing at high rates in both length and weight in the laboratory. Males of $L$. plei grew at a mean rate of $47 \mathrm{~mm} / \mathrm{mo}(S \bar{x}=7.6)$ and $13.3 \mathrm{~g} / \mathrm{mo}(\mathrm{S} \overline{\mathrm{x}}=5.30)$, while $L$. pealei males grew at a mean rate of $44 \mathrm{~mm} / \mathrm{mo}(\mathrm{S} \overline{\mathrm{x}}$ $=10.7)$ and $37.3 \mathrm{~g} / \mathrm{mo}(S \bar{x}=10.94)$. In contrast, adult females grew little or not at

TABLE IV

Laboratory growth of male and female Loligo plei (top) and Loligo pealei (bottom)

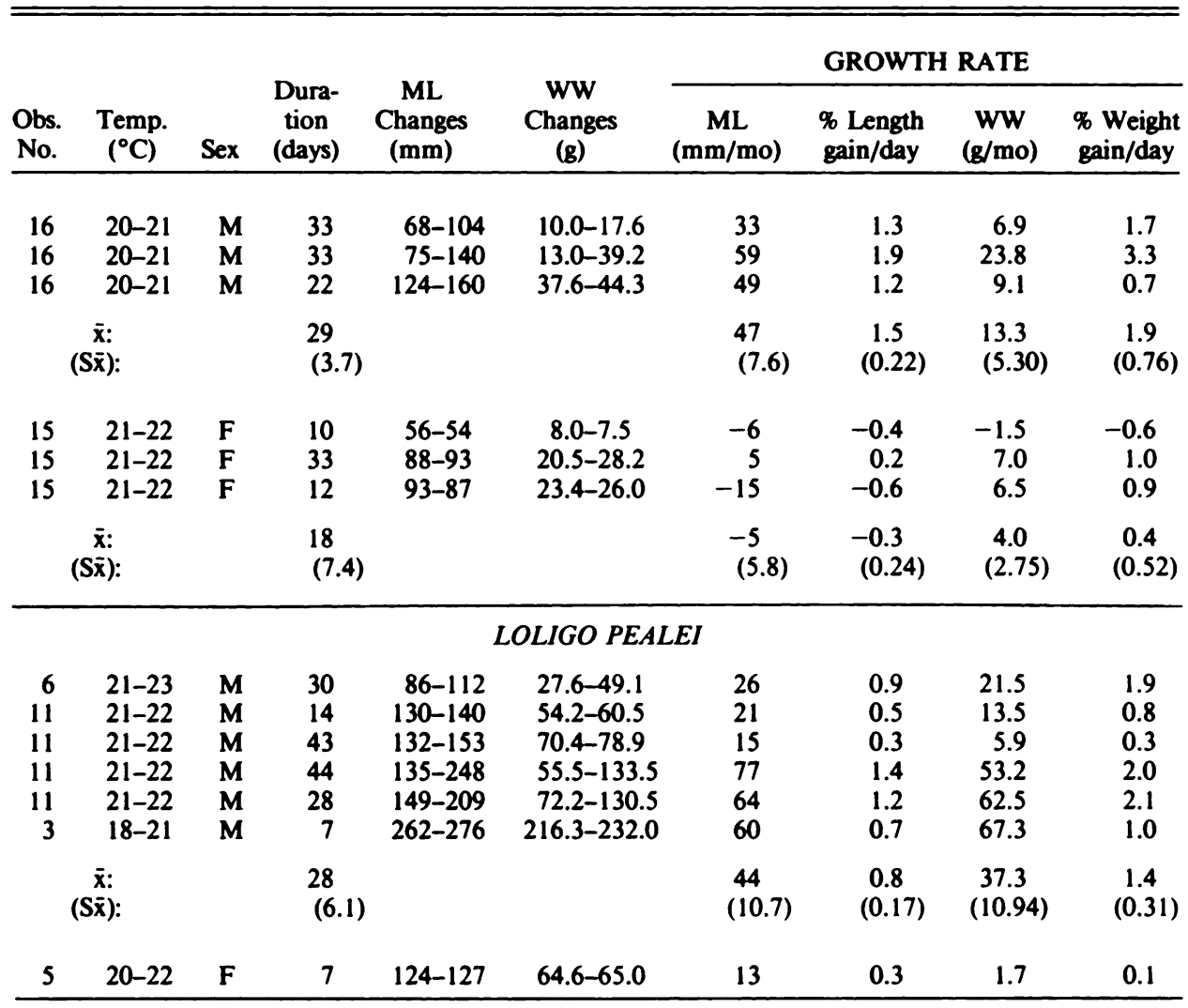




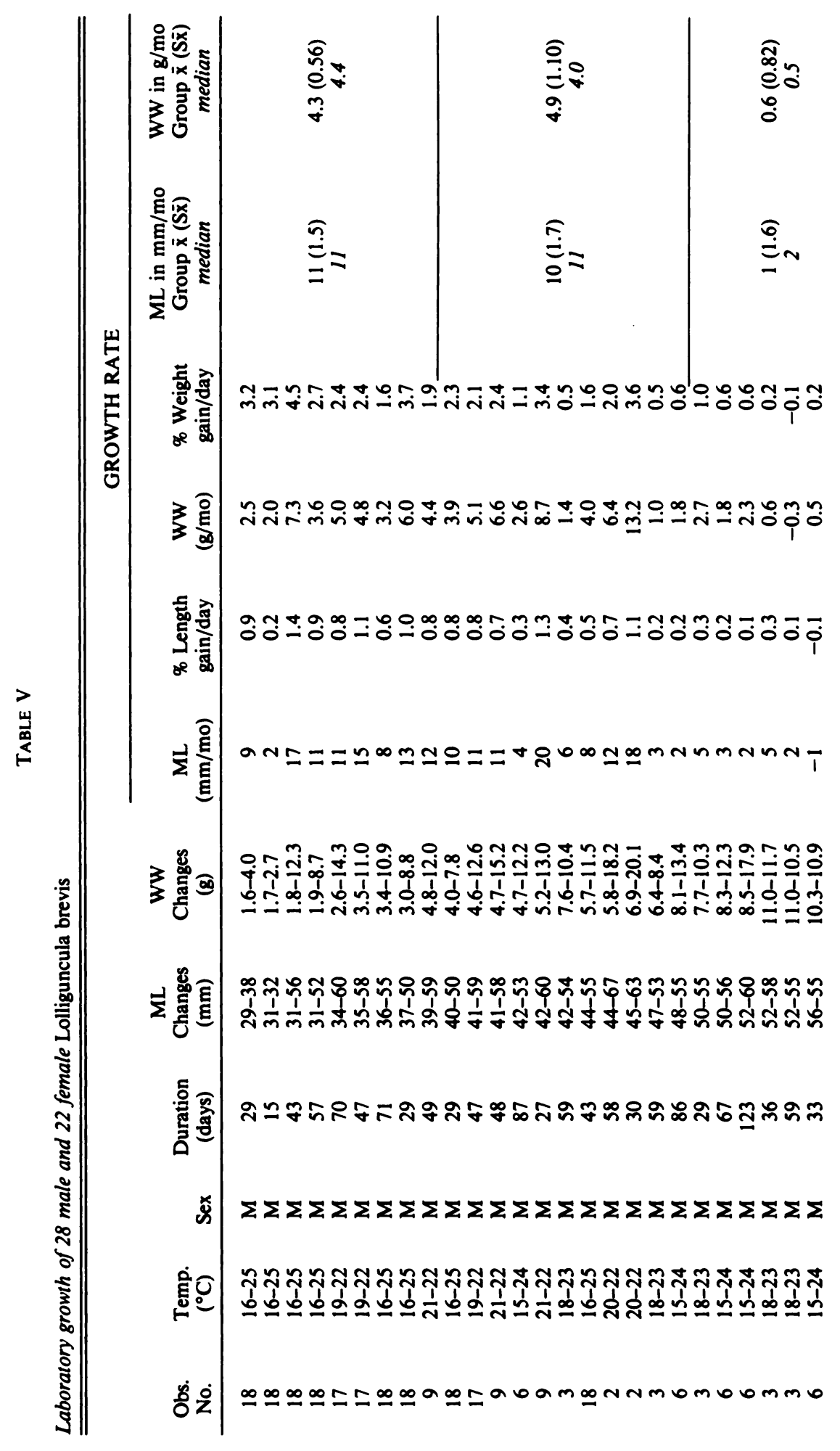




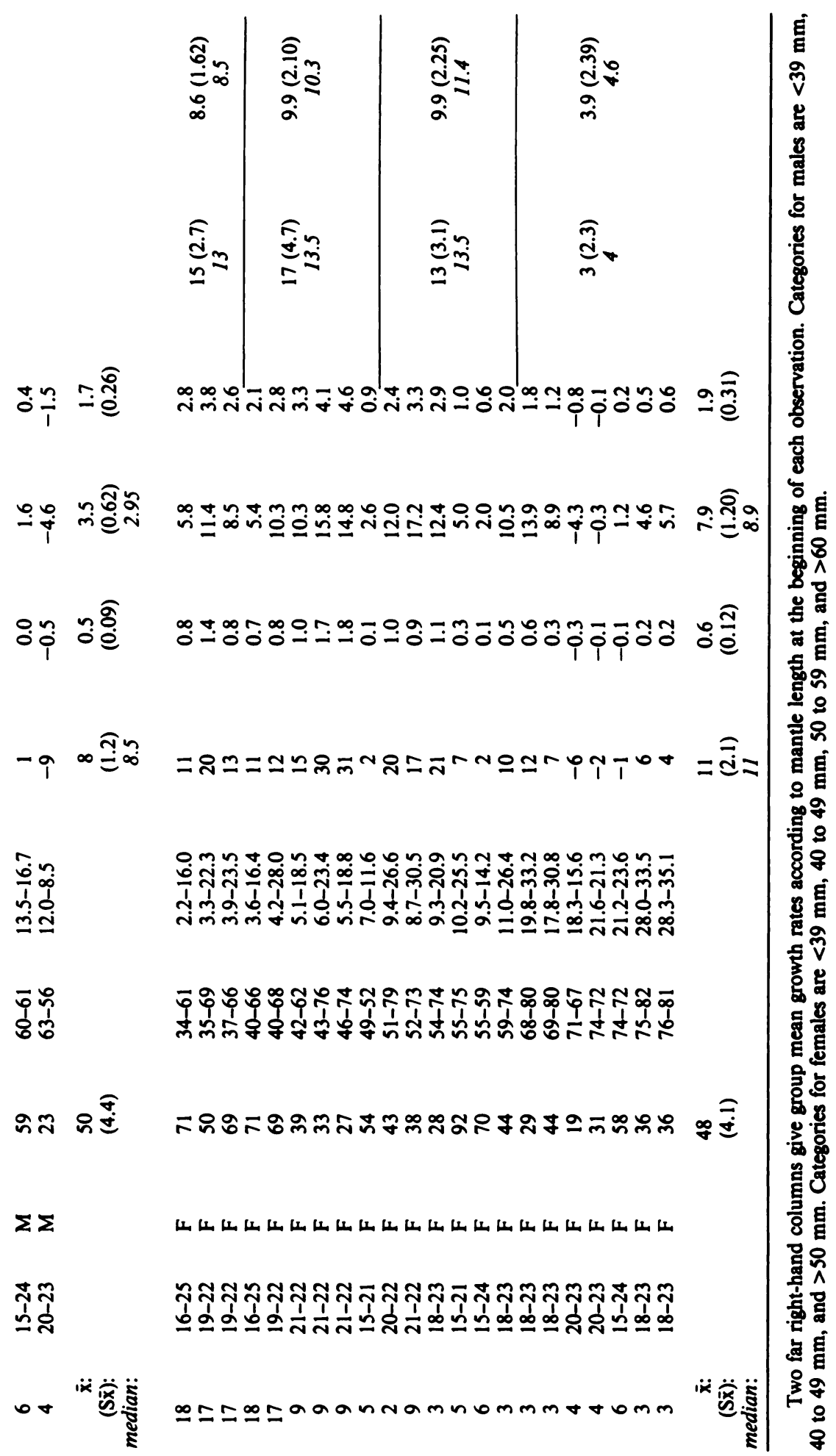


all in these observations; negative mantle length values resulted from damage to the posterior mantle during confinement. All females were mature when captured and their oviducts were full of eggs when death occurred. Unfortunately, no growth observations on juvenile Loligo were made.

Growth observations were obtained on 28 male and 22 female Lolliguncula brevis; temperatures ranged from 15 to $25^{\circ} \mathrm{C}$ (Table V). Males and females survived equally well during these observations; overall mean survival was 50 days $(S \bar{x}=4.4)$ for males and 48 days $(S \bar{x}=4.1)$ for females. Males grew in length at an overall mean rate of $8 \mathrm{~mm} / \mathrm{mo}(\mathrm{S} \overline{\mathrm{x}}=1.2)$ and $3.5 \mathrm{~g} / \mathrm{mo}(\mathrm{S} \overline{\mathrm{x}}=0.62)$, while equivalent rates for females were $11 \mathrm{~mm} / \mathrm{mo}(S \bar{x}=2.1)$ and $7.9 \mathrm{~g} / \mathrm{mo}(S \bar{x}=1.20)$. There were no statistically significant differences in median monthly growth rates in length between the sexes (males $8.5 \mathrm{~mm} / \mathrm{mo}$, females $11 \mathrm{~mm} / \mathrm{mo})$, but males $(2.95 \mathrm{~g} / \mathrm{mo})$ differed significantly from females $(8.9 \mathrm{~g} / \mathrm{mo})$ in median monthly growth in weight $(.01>P>.001)$. These weight differences reflect the maturation of reproductive organs and the production of eggs in adult females.

In both sexes of Lolliguncula brevis there were size-dependent differences in growth rate. Small young squids grew faster than larger (and presumably older) adults. Males were divided into three categories ( $<39 \mathrm{~mm}$ ML, 40 to $49 \mathrm{~mm}$ ML, $>50 \mathrm{~mm}$ ML) based on their mantle length at the beginning of the growth observation (Table $V$ ). Males $>50 \mathrm{~mm}$ ML grew in length at a mean rate of only $0.9 \mathrm{~mm} / \mathrm{mo}(\mathrm{S} \overline{\mathrm{x}}=1.6)$ compared to $10 \mathrm{~mm} / \mathrm{mo}(S \bar{x}=1.7)$ for the 40 to $49 \mathrm{~mm} \mathrm{ML}$ group and $11 \mathrm{~mm} /$ mo $(S \bar{x}=1.5)$ for the $<39 \mathrm{~mm}$ ML group. Similar differences in monthly growth rates in wet weight were measured (Table V). The median monthly growth rate in length of the $>50 \mathrm{~mm} \mathrm{ML}$ group $(2 \mathrm{~mm} / \mathrm{mo})$ differed significantly $(P<.05)$ from the median growth rates of the other two groups (both $11 \mathrm{~mm} / \mathrm{mo}$ ). The same statistical results among the three groups were obtained using the monthly growth in weight measurements. The reason for this reduced growth rate is that males $>50 \mathrm{~mm} \mathrm{ML}$ are nearing maximal size and the end of their life cycle.

Female Lolliguncula brevis were grouped into four categories using the same criterion: $<30 \mathrm{~mm}$ ML, 40 to $49 \mathrm{~mm} \mathrm{ML}, 50$ to $59 \mathrm{~mm} \mathrm{ML}$ and $>60 \mathrm{~mm} \mathrm{ML}$ (Table V). The mean monthly growth rate in length of the $>60 \mathrm{~mm}$ ML group was only $3 \mathrm{~mm} / \mathrm{mo}(S \bar{x}=2.3)$ compared to over $13 \mathrm{~mm} / \mathrm{mo}$ for the other three groups. However, there was only a statistically significant difference $(.10>P>.05)$ between the median monthly growth in length of the $>60 \mathrm{~mm} \mathrm{ML}$ group $(4 \mathrm{~mm} / \mathrm{mo})$ and the 40 to $49 \mathrm{~mm}$ ML group (13.5 mm/mo). Similar results were obtained using the monthly wet weight data from the four groups. The mean monthly increase in wet weight of the $>60^{\circ} \mathrm{mm} \mathrm{ML}$ group was low (mean $3.9 \mathrm{~g} / \mathrm{mo}, \mathrm{S} \overline{\mathrm{x}}=2.39$ ) compared to the other three groups which were all above $8.6 \mathrm{~g} / \mathrm{mo}$. However, the median monthly growth rates in weight among the four groups were not statistically different. Females showed reduced growth rates beyond $60 \mathrm{~mm}$ ML because they, like males, were reaching maximal size.

The growth measurements suggest that the three species generally grow in the laboratory at similar instantaneous relative growth rates (Tables IV and V); comparisons among species of differing sizes are best done using instantaneous relative growth rates (percent gain per day). Female Loligo plei and female Loligo pealei are not included due to the reasons mentioned earlier. The mean instantaneous relative growth rates in weight ranged from $1.4 \% /$ day for $L$. pealei males to $1.9 \% /$ day for L. plei males and $1.9 \%$ /day for Lolliguncula brevis females; the highest measured rate was $4.6 \% /$ day for a Lolliguncula brevis female. The mean instantaneous relative growth rates in length were $0.8,0.5$, and $0.6 \%$ /day for $L$. pealei males, and Lolliguncula brevis males and females, respectively. The mean instantaneous relative growth rate 
for $L$. plei males was $1.5 \% /$ day, but since this is based on only three squids it is difficult to make comparisons. The highest measured rate was $1.9 \% /$ day for a L. plei male.

Gross growth efficiency (GGE) was estimated separately for three males and three females of Loligo plei. All squids had been maintained previously for 42 days in CT systems. At $21^{\circ} \mathrm{C}$, the three males $(107,136,136 \mathrm{~mm} \mathrm{ML})$ collectively gained 12.5 $\mathrm{g}$ over six days while ingesting $56.9 \mathrm{~g}$ of fish, for an estimated 22 percent GGE. However, one male was dominant and very aggressive, and he was taking the vast majority of food and accounted for $12.3 \mathrm{~g}$ of the weight gain. With a conservative estimate that he obtained 80 percent of the fishes, his GGE was 27 percent, and his daily food intake was 18 percent of his body weight per day. The three females (56, $89,95 \mathrm{~mm} \mathrm{ML}$ ) were sexually mature and full of eggs. Collectively they gained only $1.4 \mathrm{~g}$ in six days while ingesting $36.1 \mathrm{~g}$ of fish, for an estimated 4 percent GGE. These females were eating five to 17 fishes each day, and their collective mean daily food intake was 10 percent. Apparently, either 10 percent daily food intake represents the females' required maintenance ration, or egg production utilized most of the energy that otherwise may have contributed to somatic growth.

\section{Principal causes of mortality}

The majority of deaths have been related to (1) fin damage, (2) intraspecific aggression, (3) sexual maturation, mating, and subsequent egg laying by females, and (4) crowding.

Fin damage (Fig. 7) was very critical because it impaired normal swimming and hovering and it eliminated stabilization during jet-propulsed movements, which were necessary for deftly pursuing and attacking prey and avoiding aggressive conspecifics. Details of the effects of fin damage were reported elsewhere by Leibovitz et al. (1977) and Hulet $e t$ al. (1979). Although survival during shipboard transport and laboratory transfer was fairly good, injuries incurred during shipboard transport of all species and during trawl capture of Lolliguncula brevis often accounted for many deaths during the first few days in captivity. Shipboard movement during heavy weather and long transports caused increased wall contact that resulted in skin abrasion to the squids, especially smaller ones.

The cumulative effects of fin damage from sporadic wall contact during long maintenance periods also contributed to mortality in all species. There were rare cases in which minor fin damage healed in some squids. Usually, however, the damage remained in a steady state or slowly spread from bacterial infection. Subsequent wall contact exacerbated existing wounds until eventually the fins became useless. The patterns painted on the walls apparently helped reduce wall contact, but they did not eliminate it.

Intraspecific aggression was one primary cause of mortality once the squids were in the laboratory. It was characteristic among Loligo plei males and, to a slightly lesser degree, Loligo pealei males; Lolliguncula brevis did not show obvious signs of aggression. During establishment of their rank order and during mate selection, the males vigorously made lateral displays and frontal attacks on subordinate males and sometimes females. This disrupted feeding and led to increased fin damage from wall contact when subordinate squids escaped. If Loligo spp. squids of a large size difference were put in the same tank, the smaller squids were nearly always badly harassed and died from fin damage and/or starvation within days, and on occasion they were cannibalized.

Sexual maturation and its manifestations were another primary cause of mortality. From the standpoint of laboratory survival, mating in Loligo was a fatal event because 
females usually laid eggs and died within a few days. After repeated matings, males of Loligo plei occasionally underwent an apparent catabolic change in which the arms and fins deteriorated until the squids could not swim or capture food. Females of Lolliguncula brevis (42 to $99 \mathrm{~mm} \mathrm{ML)}$ and Loligo plei (51 to $139 \mathrm{~mm} \mathrm{ML)}$ often showed very rapid sexual maturation and egg development within two to three weeks in captivity. Lolliguncula brevis and Loligo plei females that were segregated from males often produced so many eggs that the mantle bulged and the internal organs were pushed forward, probably affecting digestion; they would often die without laying eggs.

Crowding caused increased intraspecific aggression, fin damage from more frequent contact with the wall, and disruption of feeding. Had crowding been allowed over long periods, it would have resulted in deterioration of water quality if the biological carrying capacity of the tank system were exceeded.

There are other factors that contributed to mortality. Loligo plei that inked during transfer in plastic bags died quickly in the inky water. Another similar event, which we called the "shock syndrome," occurred when $L$. plei squids were startled and began to ink. However, the ink was only ejected into the mantle and over the gills, but not forcibly enough to get it out of the mantle. Ventilatory movements ceased immediately and the squids invariably died. On rare occasions Loligo spp. would leap completely out of the tank during the night. As previously mentioned, cannibalism by Loligo spp. accounted for some mortality. A certain number of deaths were inexplicable, i.e., there was no skin damage, no aggression, etc. It is possible that an inconspicuous pathologic condition existed, that parasites weakened the squids, or that there was a nutritional deficiency. Although these were not obvious, they deserve future attention.

A typical scenario of how fin damage, aggression, and sexual maturation affected survival in a typical summer experiment on Loligo plei is as follows. Out of 20 adult squids (ten male, ten female) caught at a night lighting station, 17 would survive to dockside during a seven-hour transport. Within the first five days in a CT system, four squids would die as a result of fin damage incurred during capture and transport. The other 13 squids would school together and feed well for the next two weeks except for isolated and mild aggression by the largest male as he established and maintained rank order. During this time the size of the females' ovaries and nidamental glands would swell noticeably and the accessory nidamental gland would become bright red. Pair formation would begin, with the large male herding two to three females from the school and laterally displaying towards subordinate males, who would begin to accrue fin damage from hitting the walls during escape. Two males would die within two days of this (about Day 18). Mating by several pairs would take place over several days; five females would lay eggs within one day and then die (about Day 21). Intraspecific aggression would increase, two males would die from repeated matings or fin damage, rank order would change, and several more matings with egg laying would occur by Day 25 . Conditions would briefly stabilize for the remaining four squids. Then another three would die within one week-one female from having too many eggs but not laying them, and two males from aggression and fin damage - until only one large male remained alive for several more weeks (Day 50). Mean survival would be about 20 days.

\section{Discussion}

Our results clearly demonstrate that successful transport and long-term maintenance of live loliginid squids are strongly dependent upon avoiding damage to the 
skin and fins during capture, and upon using sufficiently large tanks during laboratory maintenance to sustain high quality sea water. These points cannot be overstressed. Key factors for laboratory survival may be summarized as follows: (1) prevention of skin abrasion during capture, transport aboard ship, and transfer to the laboratory, (2) the tank system must be sufficiently large, with opaque walls and preferably no corners, (3) water quality must be high, (4) squids must have an ample food supply, (5) they must not be crowded, (6) only squids of similar size should be in the same tank to reduce aggression and cannibalism, and (7) sexes should be segregated to reduce aggression associated with courtship, mating, and egg laying.

\section{Capture and transport}

From the outset we recognized that capturing a live, undamaged squid is difficult. Over the past five years we experimented with several capture strategies: trawls, dipnets and squid jigs with night lights, and encirclement nets such as lampara nets and purse seines. Trawling is the least satisfactory capture method because of the high percentage of dead and damaged squids due to prolonged contact with the net or other animals, and to dropping of the catch on deck, which is a common practice of fishermen. Trawling is the capture method presently used to capture squids for physiological work at Woods Hole (Summers, 1968, 1969; Summers and McMahon, 1970, 1973; Summers et al., 1974), at Plymouth, England (Holme, 1974) and in the past off Ocean City, Maryland (Brinley and Mullins, 1964). Few of the squids reach shore alive because of skin damage, and those that do live stay alive briefly or for only a few days (Holme, 1974). We have tested five trawl nets, but during 226 trawl stations we had very little success in capturing live undamaged Loligo spp. in depths between 20 and $200 \mathrm{~m}$. These nets have increased our catch of live Lolliguncula brevis, and for this species trawling is our primary collection method. Success with Lolliguncula brevis is mostly attributable to the short-duration tows in very shallow water, less than $10 \mathrm{~m}$ deep. From our experience and that of many others, it appears that trawl capture of large Loligo spp. from deep water may not ever by a satisfactory collection technique if squids are to be kept alive more than a few days.

Less traumatic capture methods include squid jigging (day or night) or attracting squids to lights and either dipnetting them, jigging them, or encircling them with a lampara net or purse seine. Unfortunately, the mean catch rate has been low, primarily because of the inconsistent attraction of squids to lights. It is likely that there is a species-specific response to light and that a host of other factors such as hydrographic conditions, moon phase, food availability, and sexual condition can influence squid behavior in relation to artificial light. These parameters are not well defined for our species. In some other geographic areas, squids may be caught alive with these methods or with pound nets or floating fish traps, and these are certainly the preferred methods if long-term maintenance is a key objective (Tardent, 1962; Summers and McMahon, 1970; Flores et al., 1976, 1977; Matsumoto, 1976; O’Dor et al., 1977; Hurley, 1978; Matsumoto and Shimada, 1980). Without doubt, future work on improving light attraction and atraumatic capture methods that impart little or no skin damage should receive high priority because it affects all aspects of squid maintenance.

Factors affecting survival during shipboard transport have been discussed in Results. We believe that the configurations of the HCT and RHT tanks and their recommended stocking densities provide adequate transport survival if the squids are in good condition and water quality is not allowed to deteriorate. The larger the volume of the tanks, the better, but vessel size will limit this in most cases. For comparison, Flores et al. $(1976,1977)$ reported that fishermen transported 1000 Todarodes pacificus in shipboard 
live wells of 40001 capacity for about 12 hours, but they noted that the extreme crowding ( 1 squid/4 l) resulted in extensive fin damage to most squids. O'Dor $e t$ al. (1977) transported 20 Illex illecebrosus per container $(60 \times 90 \times 30 \mathrm{~cm}$ deep, or 1 squid/8 1), but because of the short transport time of one hour, no mortalities occurred. Matsumoto (1976) transported 15 Doryteuthis bleekeri in a $1 \times 1 \times 1 \mathrm{~m}$ tank (1 squid/66 l) for 3 to 5 hours with no mortalities; this is more space per squid than our recommendation of ten Loligo spp. per 5801 HCT tank (1 squid/58 1). These results verify that squids cannot be crowded during long transport.

In many operations, a major breakdown in the successful handling of live squids takes place at dockside. Our method of placing squids in plastic bags eliminates many of the problems encountered at this stage, especially sloshing water that led to skin damage and external commotion that startled the squids. Flores et al. (1976) used a similar method that worked equally well. It would be desirable to reduce as much as possible the large salinity and temperature shocks that squids encounter during laboratory transfer, but this is often impractical.

\section{Sea water systems}

The performances of the $2 \mathrm{~m}$ circular tank systems and the 10,000 1 raceways were satisfactory. Both designs provided two essential criteria: the capability to sustain high quality water, and the physical dimensions to accommodate the movements and habits of the squids. The advantages of our closed sea water systems are (1) independence from a natural sea water supply and hence, reproducibility at inland laboratories, (2) efficient filtration of recirculated water, (3) large volume and wide horizontal space for distribution of squids, (4) accessibility to and observation of live animals, (5) simple construction, and (6) low cost.

We chose a closed (recirculating) system over an open (flow through) system for several reasons. Water quality adjacent to Galveston Island is variable and often unsatisfactory. Closed systems offer better control over temperature and salinity fluctuations, disease organisms, turbidity, pollutants, and undesirable animals that compete with cultured organisms for space and nutrients (Spotte, 1979a). Moreover, once the requisite conditions for each species are identified, they can be carefully and continuously regulated. It is clear that appropriately designed closed systems are suitable for squid maintenance, since a comparison of squid maintenance work done in open versus closed systems showed that maintenance success with closed systems equaled or surpassed that in open systems (Boletzky and Hanlon, 1983).

Water quality is of great importance. Artificial sea water is a satisfactory substitute for natural sea water, as evidenced from our present results and our success in rearing Loligo opalescens from hatching to adult size over an 8-month period (Hanlon et al., 1979; Yang et al., 1983). Aside from its biological usefulness, we found it to be as cost effective as natural sea water because of the ship and personnel time required to obtain high salinity offshore water, and the time and space needed to filter and store it. Buildups of inorganic nitrogen (ammonia, nitrite, nitrate) were not particularly high in our systems and were not a probable cause of mortality. However, our detailed chemistry tests were few, and the subject of nitrogen tolerance is critical to closed system maintenance and culture. Since 1982, we have had detailed chemistry tests performed weekly on all systems. Preliminary results from transport experiments of Lolliguncula brevis in plastic bags (one squid per 41 of sea water) indicate strongly that they die primarily from decreased $\mathrm{pH}$ (increased hydrogen ion concentration) and secondarily from ammonia buildup. For example, if $\mathrm{pH}$ is maintained within \pm 0.2 of its original level (e.g., 8.0), squids can survive up to 30 hours even when 
levels of ammonia gradually increase to $10 \mathrm{mg} / 1 \mathrm{NH}_{4}-\mathrm{N}$ (or 100 times the recommended levels of Spotte, 1979a). In contrast, squids usually die if the pH is allowed to drop below about 7.0. Therefore, it seems that $\mathrm{pH}$ is probably the most important barometer of water quality for squids. Obviously, a great deal more work must be done to understand aspects of water quality that most affect squid survival.

Matsumoto (1976) and Matsumoto and Shimada (1980) are the only authors that give any filtration information on closed systems for squids. Matsumoto's first system (1976) utilized sand filtration. In an improved system (Matsumoto and Shimada, 1980) they added $20 \mathrm{~kg}$ of zeolite and $10 \mathrm{~kg}$ of crushed oyster shell to the filtration system. They attributed longer survival of squids to the zeolite, but the reasons are unclear. Zeolite is a naturally occurring porous material that removes selective ions by a combination of ion exchange and adsorption, but its use in marine systems is limited because of competition from other ions in sea water that quickly reduce the number of exchange sites available for binding contaminant ions such as ammonium, nitrate, and phosphate (Spotte, 1979a). Johnson and Sieburth (1974) examined the efficacy of zeolite in removing ammonium ions in salinities ranging from zero to 25 ppt. They found that, although initially it removed ammonium ions very efficiently, it lost its effectiveness after only two or three liters of sea water (25 ppt.) had passed through the ion exchange column. Furthermore, they found that the ideal size for granules was 1.00 by $0.35 \mathrm{~mm}$; Matsumoto and Shimada (1980) used an average diameter of $3.00 \mathrm{~mm}$. Based upon this scant information, it appears as though the use of zeolite in marine systems is limited to occasional, brief use to complement existing biological filters, but it does not seem likely that its continued use enhances filtration.

Matsumoto and Shimada (1980) did not give values for $\mathrm{pH}$ or nitrogenous buildup. However, it seems likely that improved survival of their squids was due partly to the buffering capacity of the added oyster shell rather than to zeolite. Our CT systems resulted in comparable survival using $360 \mathrm{~kg}$ of oyster shell as the only biological filtration substrate. Reports by Hirayama (1970) and Bower et al. (1981) show that sand filters (predominantly silica) have poor buffering capacities and that some calcareous filtrant (e.g., oyster shell or coral with calcium carbonate, or dolomite with calcium carbonate and magnesium carbonate) is necessary to buffer closed sea water systems. In view of these data, it is possible that the use of zeolite is unnecessary. Rather, it is more important to have a large filtering bed area of calcareous material and a small animal load, and to monitor $\mathrm{pH}$ and inorganic nitrogen buildup closely to insure high quality water.

Tank size and configuration are also important to squid maintenance. Survival is generally better in tanks with wide horizontal dimensions and no corners, all other factors being equal. The narrow rectangular tanks used by Summers and McMahon $(1970,1974)$ and Summers $e t$ al. (1974) ranged in size from $0.92 \mathrm{~m}$ wide $\times 1.83 \mathrm{~m}$ long $\times 0.31 \mathrm{~m}$ deep to $1.37 \mathrm{~m}$ wide $\times 3.66 \mathrm{~m}$ length $\times 0.31 \mathrm{~m}$ deep, and mean survival was two weeks or less. Larger round tanks from 1.5 to $2.0 \mathrm{~m}$ wide were used by a variety of investigators to improve mean survival up to two to four weeks (e.g., Neill, 1971; Matsumoto, 1976; Soichi, 1977; Hurley, 1978; Matsumoto and Shimada, 1980; the CT system in this report). Large rectangular tanks (e.g., LaRoe, 1971; Mikulich and Kozak, 1971; Flores et al., 1976, 1977; the raceway system in this report) produced similar mean survival of several weeks. Finally, the very large 15 m-diameter circular tank used by O'Dor et al. (1977) resulted in survival between 26 and 82 days.

The painted wall patterns probably reduced wall contact by the squids. However, we believe their effect was minimal on healthy, undamaged squids because they easily 
avoided the walls in white walled tanks as well. Although damaged squids or those engaged in intraspecific aggressive behavior hit walls regardless of their pattern, the painted walls seemed to result in fewer collisions. These situations argue in favor of bumper systems to lessen impact, but our impression is that this is not usually worth the logistical difficulties involved. Rather, it is more practical to keep fewer squids in larger tanks, so that the decrease in wall-to-volume ratio compensates for the bumper. We used a polyethylene bumper sloping at $45^{\circ}$ in Observation 1 for Loligo plei (Table I), but it did not noticeably enhance survival.

Several worthwhile comparisons may be made between our closed system 10,000 1 raceway and the 580,000 1 open system Aquatron used by O'Dor et al. (1977) to study Illex illecebrosus. In one sense, the Aquatron may represent the ultimate squid holding tank because its great size provides a more natural environment for aspects of normal behavior such as schooling, foraging, and reproduction. Two major drawbacks are its cost and the difficulties of recapturing squids. We believe raceway systems similar to that described herein offer a reasonable compromise. The raceways are simple in design, inexpensive, and manufactured in a variety of lengths and widths. Furthermore, squids survive well in them and are easily observed and recaptured (see Fig. 3). O'Dor et al. (1977) kept a maximum of 50 squids in the Aquatron at one time. By comparison, we kept 46 Loligo plei in a raceway for a mean survival of $\sim 20$ days and a maximum of 57 days (Observation 17, Table I). O'Dor $e$ t al. (1977) speculated that the tank diameter required to allow "relaxed" behavior in Illex illecebrosus was between 3.7 and $15 \mathrm{~m}$. Our observations of loliginid squids in our $2 \mathrm{~m}$ diameter CT system indicate this distance is less for loliginid squids. Certainly the $10 \mathrm{~m}$ long $\times 2 \mathrm{~m}$ wide raceway provided sufficient room for relaxed behavior for small numbers of all three of our species, especially in later versions of the raceway in which the central partition was removed.

\section{Behavior, survival, and growth}

In the course of initially testing the prototype sea water systems, it became apparent that the behavior of the squids provided the best evaluation of the systems. This observation led to more detailed analyses of behavior that provided feedback on how to refine the methods and systems in order to accommodate the needs of the squids for long-term maintenance. The significance of this seemingly simple philosophy for providing the basic requirements of squids tends to be overlooked by many. Clearly, aspects of behavior are the true limiting factors to survival and growth of wild-caught loliginid squids in a laboratory environment. The fin and skin damage that were often cited in this report as causes of mortality were merely manifestations of either aspects of behavior, transport in small tanks, or, in Lolliguncula brevis, trawl capture.

By carefully observing the squids it was possible to correlate body patterns of chromatophores and postures with specific aspects of behavior such as stress, calmness, aggressiveness, and precopulatory behavior (Hanlon, 1978, 1981, 1982, and in prep.). Accordingly, these clues are now used to avoid some problems before they develop. For example, it is often difficult to segregate newly-caught animals by sex. When Loligo plei males begin to show lateral displays, the squids are segregated by size and by sex, with the usual result of restoring calmness and normal feeding, which in turn promotes increased survival. However, in cases such as this, the effects are relatively short-lived, on the order of several days or weeks only.

For long-term survival and growth in laboratory tanks, intraspecific aggression and sexual maturation in Loligo spp. are the two most restrictive factors. In Lolliguncula brevis, the factor most responsible appears to be sexual maturation. Feeding in all 
species is clearly not the problem. In Loligo spp., the size relationships among squids exert a strong influence on survival. One reason is that larger squids dominate prey capture. In one 16-day growth observation period (part of Observation 16, Table IV), three males $(136,136,107 \mathrm{~mm} \mathrm{ML})$ were kept together in a CT system with a diet of only fishes (Cyprinidontidae). One of the $136 \mathrm{~mm} \mathrm{ML}$ males quickly became dominant, harassed the other two squids, and ate nearly all the fishes. During this period he grew at a rate of $51 \mathrm{~mm} \mathrm{ML} / \mathrm{mo}$, while the other two squids grew the equivalent of 9 and $-4 \mathrm{~mm} \mathrm{ML} / \mathrm{mo}$. In contrast, the latter two males had grown at rates of 73 and $48 \mathrm{~mm} \mathrm{ML} / \mathrm{mo}$ during the 20 days previous to this observation when they were in a tank with squids of initial sizes of 68,75 , and $88 \mathrm{~mm} \mathrm{ML}$. It is noteworthy that the squids were less aggressive when they were smaller and new in the laboratory. A similar effect of intraspecific aggression on feeding was reported for fishes by Peter (1979).

A more dramatic intraspecific aggressive effect of size disparity was cannibalism by Loligo spp. Cannibalism was not solely a result of food deprivation because in some cases it occurred in tanks that were stocked with food organisms. Cannibalized squids were either smaller or injured. The field observation that schools of Loligo plei usually contained squids of similar size suggests that cannibalism is a means by which size specificity is maintained and by which weakened squids are eliminated. However, when schools of mating pairs are formed, as seen by Waller and Wicklund (1968) in the Bahamas, the smaller females are readily accepted as mates. Neither ourselves nor Waller and Wicklund (1968) observed cannibalism among mates.

Sexual maturation seemed to progress at an accelerated rate in the laboratory. Our evidence is twofold: the gonads of most squids usually grew rapidly within 1 to 4 weeks in the tanks, and wild-caught females of Loligo plei generally had less well-developed gonads than females of similar size that had been captured in the same geographic area but kept in the laboratory for three weeks or so. The effects of extrinsic regulators of sexual maturation such as light (intensity and cycle), temperature, and food are not understood. It is possible that the general stress of capture and maintenance, combined with constant food availability and a different light regime, was enough to accelerate sexual maturation. In any event, even the longest-lived squids of each species were always sexually mature when they died. Our recent observation that Loligo opalescens reared through the life cycle in the laboratory all attained sexual maturation and died within eight months indicates that the effects of maturation are, at least in part, intrinsically regulated and may be difficult to control in the laboratory.

Interspecific compatibility among the three species in the laboratory had an interesting correlate in the field. Although the three species were never observed together in situ, different combinations of all three species were captured together in 15-minute trawls, including all three species in the same trawl on 11 occasions (Hixon, 1980a). The results of an interspecific association analysis (Cox, 1980 based on Cole, 1949) based on 150 trawl stations showed that there was a positive coefficient of association between Lolliguncula brevis and Loligo plei, indicating that these species are found frequently in close proximity to one another. Although the other two combinations showed negative coefficients, this was a reflection of the species' areal and depth distribution as well as temperature and salinity preferences (Hixon, 1980a). These findings do not mean that these three species co-occur in the same schools, but it does indicate that species-specific schools may co-occur in the same habitat.

In Table VI are comparisons of survival among squids maintained in the laboratory by various researchers. Direct comparisons are impossible because of the highly varying conditions surrounding each worker's geographic area, the species, time of year, 
R. T. HANLON ET $A L$.

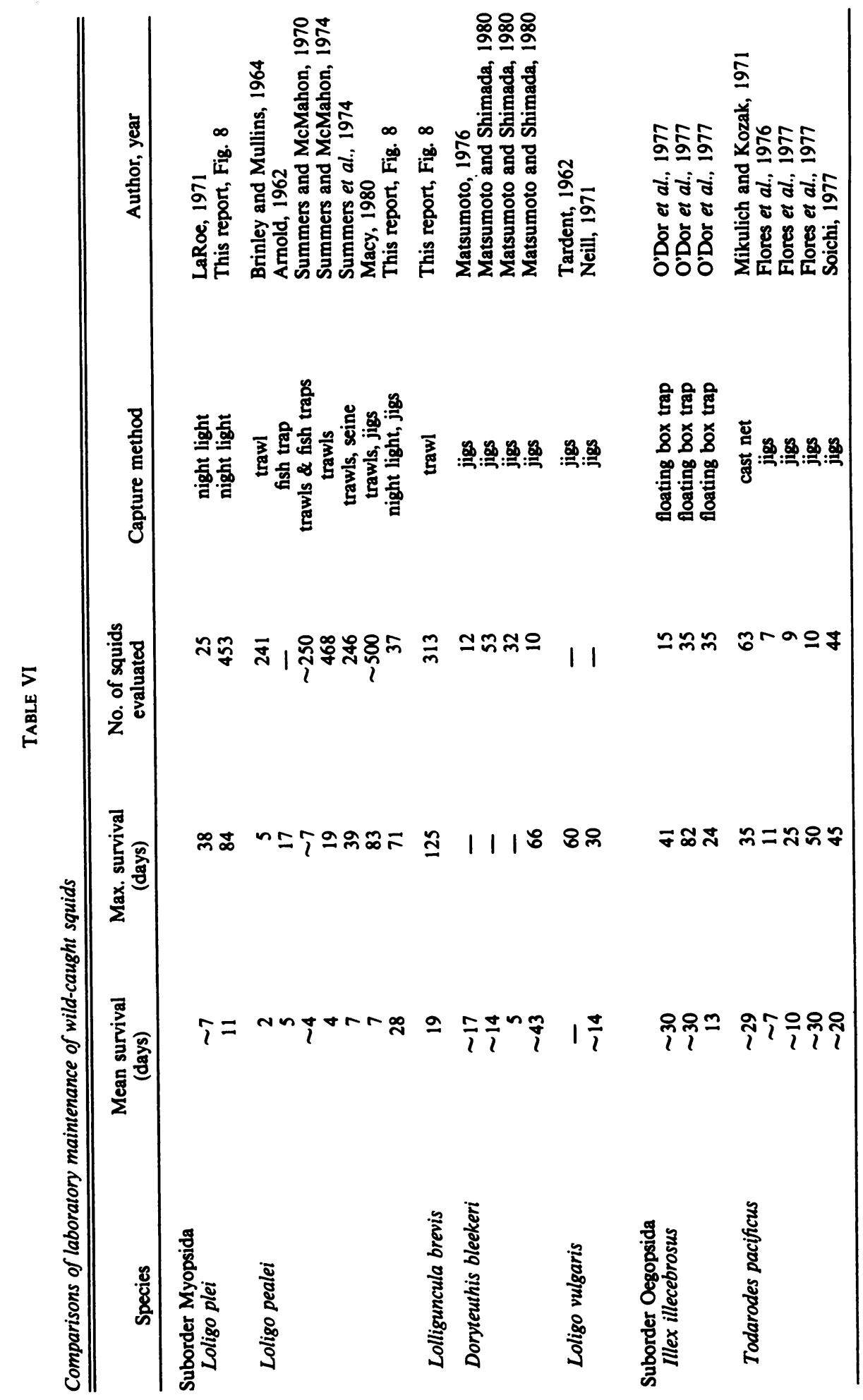


number of animals evaluated, capture and transport methods, maintenance tank size, etc. In many cases, the parameters upon which survival was described were not stated or defined clearly; more significantly, information regarding selection criteria of squids that were included or deleted from survival analyses was not always provided. Nevertheless, the table provides an overview and forms a basis for discussion. Overall, the results of our work compare favorably with other research efforts.

Survival of Loligo pealei in the laboratory (Table VI) has been very low historically. Undoubtedly, a major reason for this is that most evaluations were made on trawlcaught squids that had substantial skin damage. All of these earlier evaluations have been on $L$. pealei from New England waters. Our results of 28 days mean survival are based on few animals $(n=37)$, but they are a considerable improvement upon past efforts. The main reason for improvement is that the squids were caught in nearly perfect condition with dipnets. The very long transport times (mean 15 hours) resulted in some degree of skin damage that affected long-term survival. Seasonality had no obvious effect on survival because squids collected throughout the year survived equally well (Table II). Survival was enhanced by the fact that no juveniles were maintained, but only mid- to full-sized adults which generally do better in captivity. Survival was strongly enhanced by the bottom sitting behavior and general calmness of this species in captivity. The fallacy that bottom sitting in $L$. pealei is abnormal behavior must be dispelled once and for all. Williams (1909), Stevenson (1934), and Macy (1982) have all reported this behavior as normal, and our observations confirm their findings. From a maintenance standpoint, it may be important to provide a substrate that is suitable to the squids for bottom sitting; the crushed oyster shell in our systems was acceptable to them.

Survival of Loligo plei was fairly low overall (Table VI). This resulted partly because we analyzed every squid we caught at those stations regardless of size, sex, or condition. When conditions were good (notably Observations 11, 14, 15, 16, 17, in Table I) mean survival of squids (excluding juveniles) ranged from 14 to 84 days. This maximal survival of $\mathbf{8 4}$ days is the longest that any squid of the genus Loligo has been maintained. The steep mortality slope in Figure 8 is attributable initially to skin damage during transport and generally poor survival by juveniles, and later to intraspecific aggression and sexual maturation which limited long-term survival. We expect that long-term survival would improve by selecting only mid- or largesized squids in the best condition at capture, transporting fewer squids per tank, and segregating sexes in the laboratory.

Lolliguncula brevis survival was good. This is the only species we know of that withstands trawl capture well. As mentioned, part of the reason is the short towing period in shallow water, but this species also is apparently less susceptible to skin damage than other loliginid squids. If the high early mortality (Fig. 8) attributable to capture trauma is eliminated, then mean survival for 197 squids becomes 29 days for all sizes of squids. This compares favorably with any species studied thus far. The maximal survival of 125 days is the longest that any wild-caught squid has ever been maintained in captivity. The long survival and high growth rates of this species in captivity make it a potentially useful species for long-term in vivo experimentation.

Loligo vulgaris (from the Mediterranean) and Doryteuthis bleekeri (from Japan) may be compared best to the three species mentioned above because they are all in the Family Loliginidae. Tardent (1962) and Neill (1971) demonstrated that jig-caught Loligo vulgaris could be kept for about 14 days in large tanks. Matsumoto (1976) and Matsumoto and Shimada (1980) showed that jig-caught Doryteuthis bleekeri could be maintained consistently for about 14 days (Table VI). They also reported one run in which ten squids had a mean survival of approximately 43 days. This is 
an excellent result that, although not quantified, shows survival is high in jig-caught adult squids that are transported carefully and not crowded in laboratory tanks. Unfortunately, these authors give no details of squid size or sex, nor of the details of selection at the capture site. Therefore, it is difficult to make other comparisons between Doryteuthis bleekeri and Loligo plei (considered by some to belong to the genus Doryteuthis), which is similar in size and appearance.

Survival in captivity of the oceanic, oegopsid squids Todarodes pacificus and Illex illecebrosus has been good (Table VI). These high survival times are a result of capture and transport methods that are atraumatic, as well as the use of very large maintenance tanks and good feeding. O'Dor et al. (1977) found that mid- to large-sized adult Illex illecebrosus survived a mean of about 30 days or more, although in one group of squids mean survival was only 13 days. Mikulich and Kozak (1971), Flores et al. (1976, 1977), and Soichi (1977) reported mean survival up to 30 days for mid- to large-sized Todarodes pacificus. All of the results above are excellent examples of how squids can be kept alive for weeks if certain principals are adhered to.

To summarize the criteria necessary for good survival of squids in captivity, we once again reference the first paragraph in this discussion but also the statements by other successful researchers that reached similar conclusions (e.g., Summers et al., 1974: pg. 300; O’Dor et al., 1977: pg. 334; Flores et al., 1977). Since the squids of greatest immediate interest to neurobiologists are mainly of the genus Loligo, we believe that future researchers can expect mean survival of two weeks or more for loliginid squids captured, transported, and maintained by the methods outlined in this communication. Our demonstration that artificial sea water is a suitable substitute for natural sea water, and that a relatively simple, inexpensive closed system maintains squids well, will also provide alternate ways for others to keep squids alive for experimentation.

Growth comparisons may be made between our laboratory results and those of other researchers, and between our laboratory results and field estimates of growth. In general, all of our laboratory growth rates are higher than estimates from sizefrequency analyses of field data. Our Loligo pealei mean growth rate of $44 \mathrm{~mm} / \mathrm{mo}$ for males (Table IV) was higher than the $23 \mathrm{~mm} / \mathrm{mo}$ reported from the laboratory studies of Macy (1980) as well as the calculated field growth rate of $15.7 \mathrm{~mm} / \mathrm{mo}$ (range 6.5 to $24.5 \mathrm{~mm} / \mathrm{mo}$ ) based upon 618 males caught over a two-year period off the Texas coast (Hixon et al., 1981). Hixon et al. (1981) also provided a historical comparison of field growth rate estimates, nearly all of which are under $20 \mathrm{~mm} / \mathrm{mo}$. Our single observation of $13 \mathrm{~mm} / \mathrm{mo}$ in one female compares closely with the 11.7 $\mathrm{mm} / \mathrm{mo}$ (range 8.6 to $14.2 \mathrm{~mm} / \mathrm{mo}$ ) calculated rate of 733 females caught off the Texas coast (Hixon et al., 1981). The high growth rates in males are partly a reflection of ideal laboratory conditions, but they indicate that males are probably capable of very rapid growth in the field when conditions are favorable.

Loligo plei males grew in our laboratory at a mean rate of $47 \mathrm{~mm} / \mathrm{mo}$ (Table IV), substantially greater than the only other laboratory estimate of 15 to $25 \mathrm{~mm} /$ mo given by LaRoe (1971) for comparable temperatures. Field estimates are also lower. Whitaker (1978) estimated growth rates of 5.0 to $14.3 \mathrm{~mm} / \mathrm{mo}$ for 1065 squids caught off the southeastern U. S. during 1974 and 1975 ; the $14.3 \mathrm{~mm} / \mathrm{mo}$ rate was for a period of 132 days during spring and summer, when temperatures were similar to the laboratory temperatures in our tank systems. Hixon (1980a) calculated a growth rate of $11.5 \mathrm{~mm} / \mathrm{mo}$ (range 2.0 to $20.0 \mathrm{~mm} / \mathrm{mo}$ ) for 1819 male squids caught over a two-year period off the Texas coast. His estimate for 1887 females was $6.8 \mathrm{~mm} /$ mo (range 2.7 to $9.5 \mathrm{~mm} / \mathrm{mo}$ ); in comparison, our laboratory females did not grow (Table IV) due to sexual maturation. As in Loligo pealei, the high growth rates 
attained by males in the laboratory indicate that this species is capable of very rapid growth during brief, ideal periods.

Lolliguncula brevis males grew at a mean rate of $8 \mathrm{~mm} / \mathrm{mo}$, and females at 11 $\mathrm{mm} / \mathrm{mo}$ in our laboratory observations (Table V). No other laboratory data are available for comparison, but Hixon (1980a) estimated field growth of 1141 males at $8.6 \mathrm{~mm} / \mathrm{mo}$ (range 5.7 to $11.4 \mathrm{~mm} / \mathrm{mo}$ ), and 1045 females at $7.9 \mathrm{~mm} / \mathrm{mo}$ (range 4.3 to $12.5 \mathrm{~mm} / \mathrm{mo}$ ) off the Texas coast. Although the mean growth rates of males agree well, the maximal laboratory rate of $20 \mathrm{~mm} / \mathrm{mo}$ is much higher than the maximal field estimate of $11.4 \mathrm{~mm} / \mathrm{mo}$. Among females, both the mean $(11 \mathrm{~mm} /$ mo) and maximal $(31 \mathrm{~mm} / \mathrm{mo})$ laboratory rates are much higher than those from field estimates $(7.9 \mathrm{~mm} / \mathrm{mo}$ and $12.5 \mathrm{~mm} / \mathrm{mo}$, respectively). In all cases, Lolliguncula brevis shows the capability of growing at rates higher than previously thought when conditions are particularly good.

Some comparisons of growth in body weight may be made also. Among the three species in this study, the instantaneous relative growth rates in weight were on the same order of magnitude: males of all three species and female Lolliguncula brevis grew at mean rates of $1.4,1.7$, and $1.9 \%$ /day at temperatures of 18 to $23^{\circ} \mathrm{C}$ (Tables IV and V). In comparison, Hirtle et al. (1981) reported that Illex illecebrosus grew at rates of 1.1 to $1.9 \% /$ day at 7 to $10^{\circ} \mathrm{C}$. In the cuttlefish Sepia officinalis, Richard (1971) and Pascual (1978) reported growth rates of approximately 1.0 to $4.0 \% /$ day in mid-sized to adult animals at temperatures of 14 to $26^{\circ} \mathrm{C}$. The only other growth rate reported in the literature is by Choe (1966), who calculated a very fast rate of $7.1 \%$ /day in mid-sized Sepioteuthis lessoniana at 23 to $31^{\circ} \mathrm{C}$. Growth this fast is usually only attained by very young animals during their exponential growth phase, but apparently Sepioteuthis lessoniana is capable of continuing fast growth for a long period under ideal laboratory conditions.

The gross growth efficiency (GGE) estimate of 27 percent in a male Loligo plei and the estimated feeding rates of 18 and 10 percent for male and female Loligo plei are comparable to other squids. LaRoe (1971) reported that Sepioteuthis sepioidea ( 10 weeks old) showed GGEs of 20 to 40 percent and daily food intakes of 10 to 30 percent. Macy (1980) reported a mean daily food intake of 11 percent for adult Loligo pealei in the laboratory. Yang et al. (1983) found that laboratory-cultured Loligo opalescens had a mean daily food intake of 14.9 percent between Days 108 and 232 (adult size). Hirtle et al. (1983) reported that captive Illex illecebrosus showed a mean GGE of 40 percent and an average daily food intake of 10 percent. Soichi (1977) calculated that Todarodes pacificus had a mean daily food intake of 24.3 percent (range 10.6 to 38.9 percent).

The effects of specific diets on growth seem small. Laboratory and field studies show consistently that squids feed predominantly on crustaceans and fishes (e.g., Fields, 1965; Vovk, 1974; Ennis and Collins, 1978; Vinogradov and Noskov, 1979; Macy, 1982). In the present study, squids grew equally well on fish-only, shrimponly, or mixed diets. Hirtle et al. (1981) noted similar results with Illex illecebrosus. Previous research has indicated that smaller squids generally appear to have a slight preference for crustaceans, while fishes are preferred when the squids are larger (Hirtle et al., 1981; this report). These differences are so small that, for laboratory maintenance or growth, either diet is acceptable.

The growth results given above indicate that it is feasible to grow mid-sized squids to adult size in a reasonably short time. This may be useful for in vivo experimentation, both short- and long-term. It might also be useful as an alternate way of providing larger axons. We have already demonstrated this on a small scale in Lolliguncula brevis (Table V). With growth rates of $10 \mathrm{~mm} / \mathrm{mo}$ for mid-sized males and $13 \mathrm{~mm}$ / 
mo for mid-sized females (Table V), squids were grown another 17 to $21 \mathrm{~mm}$ over 50 days to bring them to full adult size, with axons as large as $200 \mu \mathrm{m}$ in the largest females (Hulet et al., 1980). There are possibilities with Loligo spp. as well. For example, a mid-sized Loligo plei male $100 \mathrm{~mm}$ ML could possibly be grown to 160 $\mathrm{mm} \mathrm{ML}$ in about 45 days, assuming that only the best animals were selected and that they had a sustained growth rate of $40 \mathrm{~mm} / \mathrm{mo}$. At $160 \mathrm{~mm} \mathrm{ML}$, the giant axon measures approximately $325 \mu \mathrm{m}$ in this species, sizeable enough for many types of axon experiments. The same type of operation could apply to Loligo pealei which, from our experience, is a better candidate because (1) it is less aggressive than $L$. plei, (2) it sits on the bottom, (3) it is calmer, and (4) it grows larger. Since the majority of Loligo spp. caught by night lighting off Galveston are around $100 \mathrm{~mm}$ ML, testing of this concept deserves future attention.

\section{ACKNOWLEDGMENTS}

We thank John W. Forsythe, Joseph P. Hendrix, Jr., and Deirdre A. McConathy for excellent technical assistance. We also gratefully acknowledge continued support from DHHS Grant No. RR 01024, Division of Research Resources, National Institutes of Health, and the Marine Medicine General Budget of the Marine Biomedical Institute, University of Texas Medical Branch at Galveston. Portions of this work were submitted by R.T.H. and R.F.H. in partial fulfillment of the Ph.D. requirements at the Rosenstiel School of Marine and Atmospheric Science, University of Miami, Florida.

\section{LITERATURE CITED}

ANTONIE, R. L. 1976. Fixed Biological Surfaces-Wastewater Treatment. CRC Press, Cleveland, Ohio. $200 \mathrm{pp}$.

ARNOLD, J. M. 1962. Mating behavior and social structure in Loligo pealei. Biol. Bull. 123: 53-57.

ARnold, J. M., W. C. Summers, D. L. Gilbert, R. S. Manalis, N. W. DaW, and R. J. LaSek. 1974. A Guide To The Laboratory Use Of The Squid, Loligo pealei. Marine Biological Laboratory, Woods Hole, Massachusetts. 74 pp.

BOLETZKY, S.v., AND R. T. HANLON. 1983. A review of the laboratory maintenance, rearing and culture of cephalopod molluses. Mem. Nat. Museum Victoria 44: 147-187.

BOWER, C. E., D. T. TURNER, AND S. SPOTTE. 1981. pH maintenance in closed seawater culture systems: limitations of calcareous filtrants. Aquaculture 23: 211-217.

BRINLEY, F. J., JR., AND L. J. MULLINS. 1964. The collection of squid for use at an inland laboratory. Unpublished Report to NSF (6B-1765). 6 pp.

CHOE, S. 1966. On the growth, feeding rates and the efficiency of food conversion for cuttlefishes and squids. Korean J. Zool. 9(2): 12-19. (In Korean).

COLE, L. C. 1949. The measurement of interspecific association. Ecology 30: 411-424.

CONOVER, W. J., AND R. L. IMAN. 1976. On some alternative procedures using ranks for the analysis of experimental designs. Communications In Statistics Series A5: 1349-1368.

Cox, G. W. 1980. Laboratory Manual Of General Ecology. William C. Brown, Dubuque, Iowa. 237 pp.

DiPolo, R. 1976. The influence of nucleotides on calcium fluxes. Fed. Proc. 35(14): 2579-2582.

DRAGOVICH, A., AND J. A. KeLLY, JR. 1962. A biological study and some economic aspects of squid in Tampa Bay, Florida. Proc. Gulf and Caribbean Fish. Inst. 15: 87-103.

ENNIS, G. P., AND P. W. ColuINS. 1978. Food and feeding of the short-finned squid (I. illecebrosus) during its seasonal occurrence inshore at Newfoundland and a brief review of the tropic relationships of the species. ICNAF Res. Doc. 78/11/7.

FIELDS, W. G. 1965. The structure, development, food relations, reproduction, and life history of the squid Loligo opalescens Berry. Calif. Fish \& Game, Fish Bull. 131: 1-108.

Flores, E. E. C., S. IgARAShI, R. MikAMI, AND K. Kobayashi. 1976. Studies on squid behavior in relation to fishing. I. On the handling of squid, Todarodes pacificus Steenstrup, for behavioral study. Bull. Fac. Fish. 27(3-4): 145-151.

FLores, E. E. C., S. IGARASHI, AND T. MIKAMI. 1977. Studies on squid behavior in relation to fishing. II. On the survival of squid, Todarodes pacificus Steenstrup, in experimental aquarium. Bull. Fac. Fish. 28(3): 137-147. 
GibBons, J. D. 1976. Nonparametric Methods For Quantitative Analysis. Holt, Rinehart and Winston, New York.

Hanlon, R. T. 1978. Aspects of the Biology of the Squid Loligo (Doryteuthis) plei in Captivity. Ph.D. Dissertation, University of Miami, Coral Gables, Florida.

Hanlon, R. T. 1981. Courting and mating behavior in the squid Loligo (Doryteuthis) plei. P. 325 (Abstract) in Advances in Invertebrate Reproduction, W. H. Clark, Jr., and T. S. Adams, eds. Elsevier/NorthHolland, New York. 400 pp.

Hanlon, R. T. 1982. The functional organization of chromatophores and iridescent cells in the body patterning of Loligo plei (Cephalopoda: Myopsida). Malacologia 23(1): 89-119.

HANLON, R. T., AND R. F. HixON. 1981. In situ behavioral observations of cephalopods at Grand Cayman, B.W.I. Bull. Am. Malacol. Union, 1980: 72. (Abstract)

HANLON, R. T., R. F. HIXON, AND W. H. HULET. 1978. Laboratory maintenance of wild-caught loliginid squids. Pp. 20.1-20.14 in Proceedings of the Workshop on the squid Illex illecebrosus, Fisheries and Marine Service Technical Report, no. 833, N. Balch. T. Amaratunga, and R. K. O'Dor, eds.

Hanlon, R. T., R. F. Hixon, J. W. Forsythe, AND J. P. HendRiX, JR. 1980. Cephalopods attracted to experimental night lights during a saturation dive at St. Croix, U. S. Virgin Islands. Proc. Am. Malacol. Union, 1979: 53-58.

HANLON, R. T., R. F. HixON, W. H. HULET, AND W. T. YANG. 1979. Rearing experiments on the California market squid Loligo opalescens Berry, 1911. Veliger 21(4): 428-431.

HendRIX, J. P., JR., W. H. HULET, AND J. M. GREENBERG. 1981. Salinity tolerance and the responses to hypoosmotic stress of the bay squid Lolliguncula brevis, a euryhaline cephalopod mollusc. Comp. Biochem. Physiol. 69A: 641-648.

HiRAyAmA, K. 1970. Studies on water control by filtration through sand bed in a marine aquarium with closed circulating system-VI. Acidification of aquarium water. Bull. Jpn. Soc. Sci. Fish. 36(1): 26-34.

HiRTLE, R. W. M., M. E. DeMont, AND R. K. O'DOR. 1981. Feeding, growth, and metabolic rates in captive short-finned squid, Illex illecebrosus, in relation to the natural population. J. Shell Res. 1(2): 187-192.

Hixon, R. F. 1980a. Growth, Reproductive Biology, Distribution and Abundance of Three Species of Loliginid Squid (Myopsida. Cephalopoda) in the Northwest Gulf of Mexico. Ph.D. Dissertation, University of Miami, Coral Gables, Florida.

Hixon, R. F. 1980b. Potential commercial squid resources of the Gulf of Mexico-an updated review. Pp. 54-73 in Proceedings of a Workshop for Potential Fishery Resources of the Northern Gulf of Mexico, Mississippi-Alabama Sea Grant Consortium, M. Flandorfer, and L. Skuplen, eds. Gulf Coast Research Laboratory, Ocean Springs, Mississippi. 92 pp.

Hixon, R. F., R. T. HANLON, S. M. GILlESPIE, AND W. L. GRIFFIN. 1980. Squid fishery in Texas; biological, economic and market considerations. Mar. Fish. Rev. 42(7-8): 44-50.

Hixon, R. F., R. T. HANLON, AND W. H. Hulet. 1981. Growth and maximal size of the long-finned squid Loligo pealei in the northwestern Gulf of Mexico. J. Shell. Res. 1(2): 181-185.

Holme, N. A. 1974. The biology of Loligo forbesi Steenstrup (Mollusca: Cephalopoda) in the Plymouth area. J. Mar. Biol. Assoc. U. K. 54: 481-503.

Hulet, W. H., R. T. HANLON, AND R. F. HiXON. 1980. Lolliguncula brevis-a new squid species for the neuroscience laboratory. Trends in Neuroscience 3(4): 4-5.

Hulet, W. H., M. R. Villoch, R. F. Hixon, and R. T. HaNlon. 1979. Fin damage in captured and reared squids. Lab. Anim. Sci. 29(4): 528-533.

HuRLeY, A. C. 1978. School structure of the squid Loligo opalescens. Fish. Bull. 76(2): 433-442.

JOHNSON, P. W., AND J. MCN. SiEbURTh. 1974. Ammonia removal by selective ion exchange: a backup system for microbiological filters in closed-system aquaculture. Aquaculture 4: 61-68.

Kato, S., AND J. E. HardwiCK. 1975. The California squid fishery. FAO Fish. Rep. 170. Suppl. 1: $107-$ 127.

KIER, W. M. 1982. The functional morphology of the musculature of squid (Loliginidae) arms and tentacles. J. Morphol. 172: 179-192.

LAROE, E. T. 1971. The culture and maintenance of loliginid squids Sepioteuthis sepioidea and Doryteuthis plei. Mar. Biol. 9(1): 9-25.

Leibovitz, L., T. R. Meyers, R. Elston, AND P. Chanley. 1977. Necrotic exfoliative dermatitis of captive squid (Loligo pealei). J. Invert. Path. 30: 369-376.

MACY, W. K., III. 1980. The Ecology of the Common Squid Loligo pealei Lesueur, 1821 in Rhode Island Waters. Ph.D. Dissertation, University of Rhode Island.

MACY, W. K., III. 1982. Feeding patterns of the long-finned squid, Loligo pealei, in New England waters. Biol. Bull. 162(1): 28-38.

MATSUMOTO, G. 1976. Transportation and maintenance of adult squid (Doryteuthis bleekeri) for physiological studies. Biol. Bull. 150: 279-285. 
MATSUMOTO, G., AND J. ShIMADA. 1980. Further improvement upon maintenance of adult squid (Doryteuthis bleekeri) in a small circular and closed-system aquarium tank. Biol. Bull. 159(2): 319324.

Mikulich, L. V., AND L. P. KozAK. 1971. Experimental rearing of Pacific Ocean squid under artificial conditions. Ékologiya No. 3: 94-96. (In Russian)

Mock, C. R., L. R. Ross, AND B. R. SALSER. 1977. Design and preliminary evaluation of a closed system for shrimp culture. Proc. of the 8th Annual Meeting of the World Mariculture Society. Pp. 335372.

MOYNIHAN, M., AND A. F. RODANICHE. 1982. The behavior and natural history of the caribbean reef squid Sepioteuthis sepioidea. Advances in Ethology No. 25, 151 pp.

NeILL, S. ST. J. 1971. Notes on squid and cuttlefish; keeping, handling and colour-patterns. Pubbl. Staz. Zool. Napoli 39: 64-69.

O'DOR, R. K., R. D. DURWARD, AND N. BALCH. 1977. Maintenance and maturation of squid (Illex illecebrosus) in a 15 meter circular pool. Biol. Bull. 153: 322-335.

PAINTER, H. A. 1970. A review of the literature on inorganic nitrogen metabolism in microorganisms. Water Res. 4: 393-450.

Pascual, E. 1978. Crecimiento y alimentacion de tres generaciónes de Sepia officinalis en cultivo. Inv. Pesq. 42(2): 421-442.

PETER, R. E. 1979. The brain and feeding behavior. Pp. 121-159 in Fish Physiology, Vol. 8, W. S. Hoar, D. J. Randall, and J. R. Brett, eds. Academic Press, New York.

RAND, M. C., A. E. GREENBERG, AND M. J. TARAS, eds. 1976. Standard Methods for the Examination of Water and Wastewater, 14th ed. American Public Health Association, Washington.

Rathien, W. F., R. T. HANLON, AND R. F. HixON. 1979. Squid fishery resources and development in the Northwest Atlantic and Gulf of Mexico. Proc. Gulf Carribb. Fish. Inst. 31: 145-157.

Richard, A. 1971. Contribution a l'Étude Expérimentale de la Croissance et la Striation de la Coquille de Sepia officinalis $L$. (Mollusque, Cephalopode). Thèse de Doctorat d'Etat, Univ. Lille (No. 243).

ROSENBERG, P. 1973. The giant axon of the squid: A useful preparation for neurochemical and pharmacological studies. Pp. 97-160 in Methods of Neurochemistry, Vol. 4, R. Fried, ed. Marcel Dekker, Inc., New York, 322 pp.

RUdiN, E., R. EVERETt, JR., J. J. WeINSTOCK, AND H. M. SCHOEN. 1963. Contaminant removal from sewage plant effluents by foaming. PHS Publ. no. 999-WF-5, Public Health Service, Cincinnati, Ohio. $56 \mathrm{pp}$.

SoICHI, M., 1977. On the growth and food quantity of young common squid, Todarodes pacificus, in captivity. Dosuishi 13(4): 79-82. (In Japanese)

SOLóRzano, L. 1969. Determination of ammonia in natural waters by the phenolhypochlorite method. Limnol. Oceanogr. 14: 799-801.

SPOTTE, S. 1973. Marine Aquarium Keeping: The Science, Animals, and Art. John Wiley \& Sons, Inc., New York. 173 pp.

SpotTe, S. 1979a. Fish and Invertebrate Culture: Water Management in Closed Systems, 2nd Ed. John Wiley \& Sons, Inc., New York. 179 pp.

SpotTe, S. 1979b. Seawater Aquariums: The Captive Environment. John Wiley \& Sons, Inc., New York. $413 \mathrm{pp}$.

SteVenson, J. A. 1934. On the behaviour of the long-finned squid [Loligo pealii, (Lesueur).]. Canadian Field-Naturalist XIVIII: 4-7.

Strickland, J. D. H., AND T. R. PArsons. 1972. A Practical Handbook of Seawater Analysis, 2nd Ed. Bull. 167, Fish. Res. Board Can., Ottawa, 310 pp.

Summers, W. C. 1968. The growth and size distribution of current year class Loligo pealei. Biol. Bull. 135: 366-377.

SUMmers, W. C. 1969. Winter population of Loligo pealei in the Mid-Atlantic Bight. Biol. Bull. 137: 202216.

SUMmers, W. C., AND J. J. MCMAHON. 1970. Survival of unfed squid, Loligo pealei, in an aquarium. Biol. Bull. 138: 389-396.

SUMmers, W. C., AND J. J. MCMAHON. 1974. Studies on the maintenance of adult squid (Loligo pealei). I. Factorial survey. Biol. Bull. 146(2): 279-290.

Summers, W. C., J. J. MCMAHON, AND G. N. P. A. RUPPERT. 1974. Studies on the maintenance of adult squid (Loligo pealet). II. Empirical extensions. Biol. Bull. 146: 291-301.

TARDENT, P. 1962. Keeping Loligo vulgaris L. in the Naples Aquarium. 1st International Congress on Aquarology. Bull. Inst. Oceanographique, Monaco, Special no. 1A: 41-46.

TASAK, I. 1982. Physiology and Electrochemistry of Nerve Fibers. Academic Press, New York. 343 pp.

VINOGRADOV, V. E., AND A. S. Noskov. 1979. Feeding of the short-finned squid, Illex illecebrosus, and long-finned squid, Loligo pealei, off Nova Scotia and New England, 1974-75. ICNAF Sel. Papers 5: $31-36$. 
Vovk, A. N. 1974. Feeding habits of the North American squid Loligo pealei Les. Fisheries and Marine Service, Translation Series No. 3304.14 pp.

WALLER, R. A., AND R. I. WICKLUND. 1968. Observations from a research submersible-mating and spawning of the squid, Doryteuthis plei. Bioscience 18(2): 110-111.

WhITAKER, J. D. 1978. A Contribution to the Biology of Loligo pealei and Loligo plei (Cephalopoda Myopsida) off the Southeastern Coast of the United States. Master's thesis, The College of Charleston, Charleston, North Carolina.

Williams, L. W. 1909. The Anatomy of the Common Squid. The American Museum of Natural History, New York. $91 \mathrm{pp}$.

WINBERG, G. C. 1960. Rate of Metabolism and Food Requirements of Fishes. Fish. Res. Board Can., Trans. Ser. No. 194, 202 pp.

YANG, W. T., R. T. Hanlon, M. E. KRejCI, R. F. Hixon, and W. H. Hulet. 1983. Laboratory rearing of Loligo opalescens, the market squid of California. Aquaculture 31: 77-88.

Yang, W. T., R. F. HiXon, P. E. TURK, M. E. KREJCI, R. T. Hanlon, AND W. H. Hulet. 1983. Culture of California market squid from hatching - completion of the rearing cycle to second generation hatchlings. Aquabiology. 5(5): 328-339. (In Japanese) 